$$
1 / 7^{80} \begin{aligned}
& 8^{0} \\
& \text { SAND79-2214 } \\
& \text { Unlimited Release }
\end{aligned}
$$

\title{
MASTER
}

\section{NUMERICAL SOLUTIONS FOR STEADY THERMAL CONVECTION FROM A CONCENTRATED SOURCE IN A POROUS MEDIUM}

C. E. Hickox and H. A. Watts

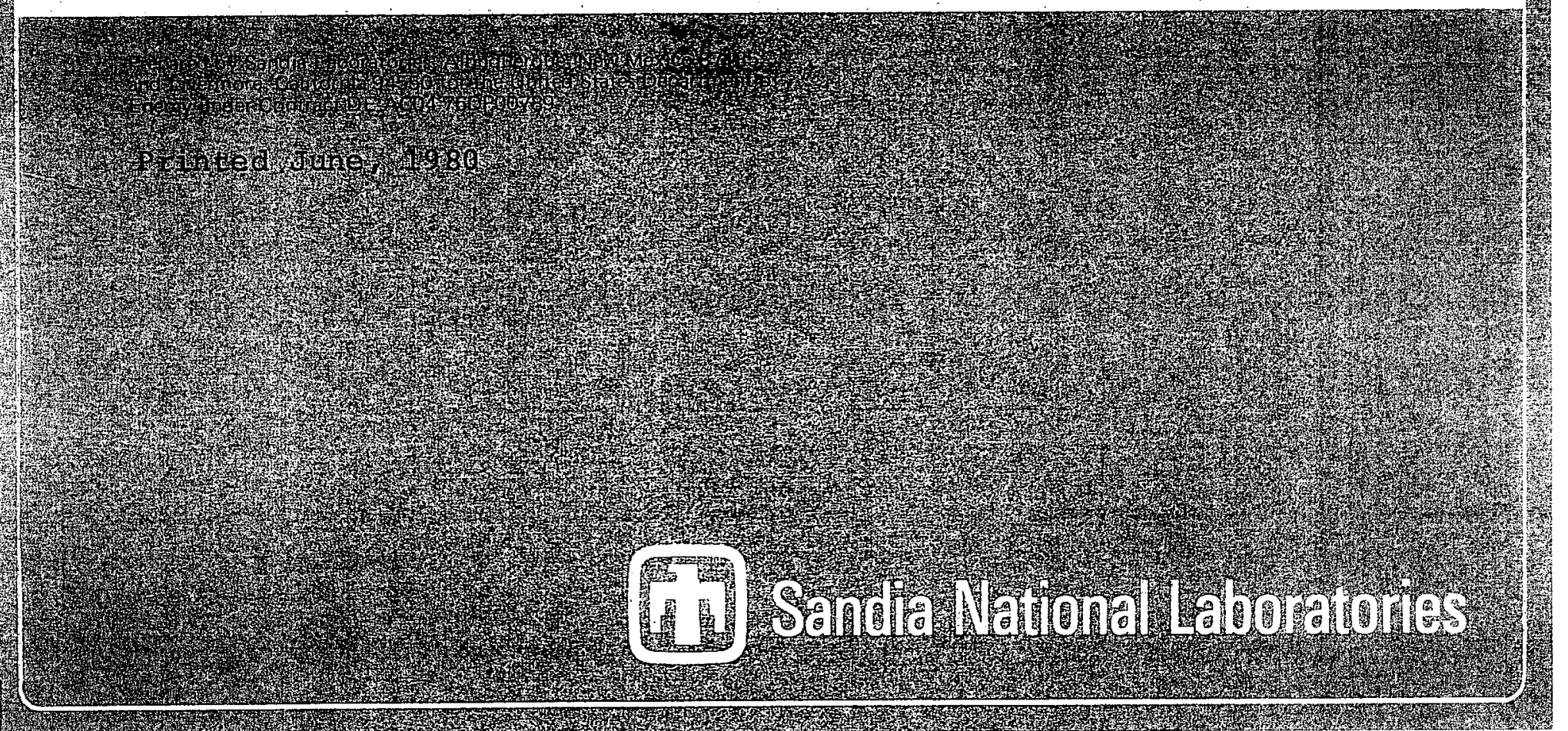




\section{DISCLAIMER}

This report was prepared as an account of work sponsored by an agency of the United States Government. Neither the United States Government nor any agency Thereof, nor any of their employees, makes any warranty, express or implied, or assumes any legal liability or responsibility for the accuracy, completeness, or usefulness of any information, apparatus, product, or process disclosed, or represents that its use would not infringe privately owned rights. Reference herein to any specific commercial product, process, or service by trade name, trademark, manufacturer, or otherwise does not necessarily constitute or imply its endorsement, recommendation, or favoring by the United States Government or any agency thereof. The views and opinions of authors expressed herein do not necessarily state or reflect those of the United States Government or any agency thereof. 


\section{DISCLAIMER}

Portions of this document may be illegible in electronic image products. Images are produced from the best available original document. 
SAND79-2214

Unlimited Release

NUMERICAL SOLUTIONS FOR STEADY THERMAL CONVECTION FROM A CONCENTRATED SOURCE IN A POROUS MEDIUM*

C. E. Hickox and H. A. Watts Sandia National Laboratories** Albuquerque, NM 87185

April 1980

\begin{abstract}
Solutions for the steady, axisymmetric velocity and temperature fields associated with a point source of thermal energy in a fluidsaturated porous medium are obtained numerically through use of similarity transformations. The two cases considered are those of a point source located on the lower, insulated boundary of a semiinfinite region and a point source embedded in an infinite region. Numerical results are presented from which complete descriptions of the velocity and temperature fields can be constructed for Rayleigh numbers ranging from $10^{-3}$ to $10^{2}$.
\end{abstract}

\footnotetext{
* This work was supported by the U.S. Department of Energy under contract DE-AC0 4-76DP00789.

${ }^{*}$ A U.S. Department of Energy facility.
} 


\section{CONTENTS}

Page

NOMENCLATURE

INTRODUCTION

GENERAL THEORY

Point Source at the Base of a Semi-Infinite Region

Point Source in an Infinite Medium

APPROXIMATE ANALYTICAL SOLUTIONS

Point Source at the Base of a Semi-Infinite Region

Point Source in an Infinite Medium

COMPUTATIONAL APPROACH

10

Point Source at the Base of a Semi-Infinite Region

Point Source in an Infinite Medium

Vertical Transport of Fluid Particles

DISCUSSION

APPENDIX A: Tabulated Numerical Results for a Point

Source at the Base of a Semi-Infinite Region

APPENDIX B: Tabulated Numerical Results for a Point Source in an Infinite Medium 


\section{Greek Symbols}

$\alpha$

B

$n$

$\theta$

$\mu$

$\rho$

$\psi$

$\zeta$

\section{$\underline{\text { Subscript }}$}

Parameter introduced in Equation (36)

Non-dimensional function related to stream function

Acceleration of gravity

Vertical transport of fluid particle

Elevation

Intrinsic permeability

Pressure

Strength of point source

Position vector in spherical polar coordinates

Rayleigh number

Cylindrical polar coordinates

Spherical polar coordinates

Temperature

Time

Velocity components in cylindrical polar coordinates Velocity components in spherical polar coordinates Velocity vector

Thermal diffusivity

Coefficient of volumetric thermal expansion

Similarity variable

Non-dimensional function related to temperature

Dynamic viscosity

Density

Stream function

Integration variable

Refers to reference conditions far from point source 


\section{INTRODUCTION}

In a previous paper, wooding ${ }^{1}$ demonstrated the utility of simple models for the description of geothermal flow processes. Of particular interest in the present context, Wooding considered the case of a point source of thermal energy located on the lower boundary of a semi-infinite, fluid-saturated, porous medium. When vertical gradients of velocity and thermal conduction were negligible compared with horizontal gradients, and the Boussinesq approximation invoked, Wooding was able to show that similarity solutions for planar or axisymmetric flow could be obtained by direct analogy with the solutions given by Schlichting ${ }^{2}$ for planar or axisymmetric, incompressible, laminar jets. The approximations imposed were, generally speaking, valid only for sufficiently large values of the Rayleigh number.

Since point source solutions can be of considerable practical utility, a more complete analysis, which requires no assumptions regarding the relative magnitudes of horizontal and vertical gradients, is developed in the present paper. Two cases, both concerned with axisymmetric flow and valid for any value of the Rayleigh number, are considered. In the first instance, the physical arrangement is identical to that considered by wooding. This case was the subject of an earlier Sandia Laboratories report ${ }^{3}$ and is included here for completeness. Also, additional results for this geometry are contained in the present report and are presented in a more useful format than those previously available. The second case treats the problem of axisymmetric flow induced by a point source embedded in an infinite, fluid-saturated, porous medium. Both cases are analyzed through the introduction of similarity transformations resulting in sets of nonlinear ordinary differential equations which are then solved numerically in order to provide descriptions of the velocity and temperature fields. 
GENERAL THEORY

In this section, mathematical models are developed for the description of axisymmetric free convection in a fluid-saturated porous medium. The medium is assumed to be rigid, homogeneous, and isotropic and the fluid incompressible, with density changes occurring only as a result of changes in the temperature according to

$$
\rho=\rho_{\infty}\left[1-\beta\left(T-T_{\infty}\right)\right],
$$

where $\rho$ is the density, $T$ is the temperature, $\beta$ is the coefficient of thermal expansion, and the subscripts refer to reference conditions. In accordance with the usual Boussinesq approximation, density changes are accounted for only in the buoyancy term in the equations of motion. It is also assumed that the fluid and matrix are in thermal equilibrium and that the fluid motion can be adequately described by Darcy's law. Permeability, viscosity, effective thermal diffusivity, and the coefficient of thermal expansion are assumed constant and dispersion effects are neglected.

The steady-state equations of continuity, motion, and thermal transport are then

$$
\begin{gathered}
\operatorname{div} \underset{\sim}{v}=0 \\
\frac{\mu}{\mathrm{k}} \underset{\sim}{v}=-\operatorname{grad}(p+\rho g h), \\
\underset{\sim}{\mathrm{v}} \operatorname{grad} \mathrm{T}=\alpha \operatorname{div}(\operatorname{grad} \mathrm{T}),
\end{gathered}
$$

where $\underset{\sim}{\mathrm{v}}, \mathrm{k}, \mu, \alpha, \mathrm{p}$, and $\mathrm{g}$ are, respectively, the velocity vector, permeability, dynamic viscosity, effective thermal diffusivity, pressure, and acceleration due to gravity. The elevation $h$ is measured vertically upward and $g$ is oppositely directed. 
In the cases to be considered subsequently, thermal energy is released continuously at a finite rate from a point source. Hence, in the absence of any bounding surfaces which can inhibit motion, any deviation from an isothermal state will result in fluid motion. We, therefore, expect no lower limit for the occurrence of natural convective motion.

\section{Point Source at the Base of a Semi-Infinite Region}

Here, we wish to consider the axisymmetric flow induced by a point source of strength $Q$ (energy generated per unit time) situated on the lower, insulated boundary of a semi-infinite region. Cylindrical polar coordinates $(r, z)$ with associated velocity components $(u, w)$ are used in the subsequent analysis. The origin of the coordinate system is coincident with the point source, and the $z$-axis is directed vertically upward.

The basic formulation now proceeds in a straightforward manner from Equations (I) through (4). Equation (1) admits the introduction of a stream function $\psi$ defined by

$$
r u=-\frac{\partial \psi}{\partial z}, r w=\frac{\partial \psi}{\partial r}
$$

Introduction of the similarity transformations

$$
\eta=r / z, \psi=\alpha z f(\eta), T-T_{\infty}=\frac{\mu \alpha}{\rho_{\infty} g k \beta} \frac{\theta(\eta)}{z},
$$

as suggested by Yih, 4 allows Equations (1) through (4) to be reduced to the set of nonlinear ordinary differential equations

$$
\begin{gathered}
\left(\eta^{3}+\eta\right) f^{\prime \prime}-f^{\prime}=\eta^{2} \theta^{\prime}, \\
-(f \theta)^{\prime}=\left(\theta^{\prime} n\right)^{\prime}+\left(n^{2} \theta\right)^{\prime}+\left(\eta^{3} \theta^{\prime}\right)^{\prime},
\end{gathered}
$$

where primes denote differentiation with respect to $n$. An additional requirement obtained upon integration of Equation (4) over a plane taken normal to the $z$-axis is

$$
\int_{0}^{\infty}\left(f^{\prime} \theta+\eta \theta+\eta^{2} \theta^{\prime}\right) d \eta=R a
$$


i

where $\mathrm{Ra}$ is the Rayleigh number

$$
\mathrm{Ra}=Q \mathrm{gk} \beta / 2 \pi \alpha^{2} \mu \mathrm{C},
$$

and $\mathrm{c}$ is the specific heat of the fluid. Physically, Equation (9) shows that the net energy flux is constant for any plane taken normal to the vertical axis. For future reference, the relationships between velocities and similarity parameters are

$$
u=\frac{\alpha}{z} \eta\left(\frac{f}{\eta}\right)^{\prime}, w=\frac{\alpha}{z}\left(\frac{f^{\prime}}{\eta}\right)
$$

From physical arguments, it is expected that $u, w$, and $\left(T-T_{\infty}\right)$ should approach zero for large $r$. In addition, $u$ as well as the radial gradients of $w$ and $T$ should be zero along the axis of symmetry. These requirements yield the boundary conditions

$$
\begin{aligned}
& f(0)=0=\theta^{\prime}(0), \\
& f^{\prime}(\infty)=0=\theta(\infty) .
\end{aligned}
$$

Solution of Equations (7) and (8) subject to the integral relation and boundary conditions given by Equations (9) and (12) will provide a complete description of the thermal and flow fields.

\section{Point Source in an Infinite Medium}

In order to analyze the axisymetric flow field induced by a point source in an infinite medium, it is appropriate to adopt spherical polar coordinates $(R, \Phi)$ with associated velocity components $\left(v_{R}, v_{\Phi}\right)$. The angle $\Phi$ is measured from the vertical axis (z-axis as introduced in the previous section) to the radial position vector $\underset{\sim}{\mathrm{R}}$. The velocity component $v_{R}$ is in the direction of $\underset{\sim}{R}$. The other velocity component $v_{\Phi}$ is normal to $\underset{\sim}{\mathrm{R}}$, lies in the plane of $\mathrm{R}$ and $z$, and is positive in the direction of increasing $\Phi$. The origin of coordinates is again taken coincident with the point source.

The formulation for this case, in essence, parallels that presented in the first part of this section. A stream function $\psi$ is defined such that

$$
v_{R}=\frac{1}{R^{2} \sin \Phi} \frac{\partial \psi}{\partial \Phi}, v_{\Phi}=\frac{-1}{R \sin \Phi} \frac{\partial \psi}{\partial R}
$$


Based on certain apparent similarities between the present system of equations and those derived by squire ${ }^{5}$ in his study of the round laminar jet, we introduce the following substitutions

$$
\eta=\cos \Phi, \psi=\alpha \operatorname{Rf}(\eta), T-T_{\infty}=\frac{\mu \alpha}{\rho_{\infty} g k \beta} \frac{\theta(\eta)}{R} .
$$

The original system of partial differential equations can now be reduced to the system of ordinary differential equations

$$
\begin{gathered}
E^{\prime \prime}=-(n \theta)^{\prime}, \\
(f \theta)^{\prime}=\theta^{\prime \prime}-\left(\eta^{2} \theta^{\prime}\right)^{\prime},
\end{gathered}
$$

where primes denote differentiation with respect to $\eta$. Integration of Equation (4) over a sphere centered about the origin provides the relation

$$
\int_{-1}^{1}\left(1-f^{\prime}\right) \theta d \eta=R a,
$$

where $\mathrm{Ra}$ is the Rayleigh number as defined by Equation (10). Velocity components are now given by

$$
v_{R}=-\frac{\alpha}{R} f^{\prime}(\eta), v_{\Phi}=-\frac{\alpha}{R}\left(1-n^{2}\right)^{-1 / 2} f(\eta)
$$

As before, it is expected that $v_{R^{\prime}} v_{\Phi^{\prime}}$ and $\left(T-T_{\infty}\right)$ should approach zero for large $R$ and that the solution should exhibit symmetry about a vertical axis. These physical requirements translate into the conditions

$$
\begin{gathered}
f(1)=0=f(-1), \\
\theta, \theta^{\prime} \text { bounded for } n= \pm 1 .
\end{gathered}
$$

Although, in some instances, the notation used in this section is in conflict with that used in the previous section, no confusion should exist so long as the two cases are considered separately. 
APPROXIMATE ANALYTICAL SOLUTIONS

When the Rayleigh number is sufficiently small, approximate analytical results can be obtained for both cases under consideration. For large Rayleigh numbers, Wooding ${ }^{1}$ obtained an approximate solution for the semi-infinite region by analogy with the results of schlichting ${ }^{2}$ for incompressible laminar jets. Presently, we know of no corresponding solution for the point source in an infinite region. The various approximate solutions are presented in the remainder of this section. These results are useful in that they provide bounds for the more general numerical results which will be presented subsequently.

Point Source at the Base of a Semi-Infinite Region

For small Rayleigh number, solutions to Equations (7), (8), and (9) can be obtained by straightforward expansion of the dependent variables in terms of the Rayleigh number. The leading terms resulting from this process provide the approximations

$$
\begin{gathered}
f=-\frac{1}{2} \operatorname{Ra}\left[\left(n^{2}+1\right)^{-1 / 2}-1\right], \\
\theta=\operatorname{Ra}\left(n^{2}+1\right)^{-1 / 2},
\end{gathered}
$$

where it may be noted that Equation (21) produces the steady-state conduction solution associated with a point source.

For a sufficiently large Rayleigh number, Wooding's analysis ${ }^{1}$ provides the results

$$
\begin{gathered}
f=\frac{3}{8} \operatorname{Ra} n^{2}\left(1+\frac{3}{32} \operatorname{Ra~} n^{2}\right)^{-1}, \\
\theta=\frac{3}{4} \operatorname{Ra}\left(1+\frac{3}{32} \operatorname{Ra~} n^{2}\right)^{-2} .
\end{gathered}
$$


Point Source in an Infinite Medium

For small Rayleigh number, the leading terms of an expansion in terms of the Rayleigh number provide the approximate results ${ }^{6}$

$$
\begin{gathered}
f=\frac{1}{4} \mathrm{Ra}\left(1-\eta^{2}\right)+\frac{1}{24} \mathrm{Ra}^{2} \eta\left(1-\eta^{2}\right), \\
\theta=\frac{1}{2} \mathrm{Ra}+\frac{1}{8} \eta \mathrm{Ra}^{2},
\end{gathered}
$$

where it may be noted that the first term of Equation (25) produces the steadystate conduction solution associated with a point source.

\section{COMPUTATIONAL APPROACH}

Although the use of similarity transformations results in simplified formulations for the cases considered, the resulting equations are still of sufficient complexity to warrant the use of numerical techniques in order to obtain general solutions. Efficient, state-of-the-art computer codes, currently under development at Sandia Laboratories ${ }^{7,8}$ and designed specifically for the solution of two-point boundary value problems, were utilized for the numerical solutions. In the remainder of this section, numerical results are presented along with brief descriptions of the techniques employed in the analysis.

Point Source at the Base of a Semi-Infinite Region ${ }^{3}$

Equations (7) and (8) can be integrated once to provide

$$
\begin{aligned}
& \left(\eta+\frac{l}{\eta}\right) f^{\prime}-f=\theta+c_{1}, \\
& -f \theta=\left(\eta^{3}+\eta\right) \theta^{\prime}+\eta^{2} \theta,
\end{aligned}
$$

where $C_{1}$ is a constant of integration, and the constant of integration associated with Equation (27) is equal to zero by virtue of Equation (12a). Further integration of the differential equations by analytical methods does not appear feasible. 
In order to obtain numerical solutions, Equations (26) and (27) could be solved subject to the boundary conditions given by Equations (12a) and (12b) for a specified value of the constant $C_{1}$. Then $C_{1}$ could be varied until the constraint condition given by Equation (9) is satisfied. However, it is desirable to automate the entire process within the framework of solving only differential equations. Thus, a differential equation for $C_{1}$ is added and Equation (9) is modified to provide

$$
S(n)=\int_{0}^{n}\left(f^{\prime} \theta+\zeta \theta+\zeta^{2} \theta^{\prime}\right) d \zeta
$$

where the integration variable is now 5 . The original boundary value problem can now be expressed by the system of first order differential equations

$$
\begin{aligned}
& \theta^{\prime}(n)=-\left(n+n^{3}\right)^{-1}\left(f+n^{2}\right) \theta, n>0, \\
& f^{\prime}(n)=n\left(1+n^{2}\right)^{-1}\left(\theta+f+c_{1}\right), \\
& C_{1}^{\prime}(\eta)=0, \\
& S^{\prime}(\eta)=n\left(1+n^{2}\right)^{-1}\left(\theta+c_{1}+1\right) \theta,
\end{aligned}
$$

subject to the boundary conditions

$$
\begin{gathered}
f(0)=0=S(0), \\
f(\infty)=-C_{1}, S(\infty)=R a .
\end{gathered}
$$

The condition $\theta^{\prime}(0)=0$ is used directly to give proper definition to Equation (29a) at the origin. Equation (29d) is obtained upon differentiation of Equation (28) and substitution from Equations (29a) and (29b). The boundary conditions on $S$ follow directly from consideration of Equations (9) and (28). The condition $f^{\prime}(\infty)=0$ is automatically satisfied by the differential equation (29b) under the assumption of boundedness for $f(n)^{-}$and $\theta(n)$. An even stronger condition emerges under our physical constraints; namely, $\lim _{n \rightarrow \infty} n f^{\prime}(\eta)=0$. The condition $\theta(\infty)=0$ in Equation (29b) leads to the new boundary condition on $f$, given by $f(\infty)+c_{1}=0$. The motivation for using this condition stems from 
difficulties encountered in attempting to obtain satisfactory numerical solutions when the condition $\theta(\infty)=0$ was imposed. It was observed that $\theta$ was driven to zero for large $n$ regardless of the acceptability of the other variables. With the alternate boundary condition, the solution algorithm was less sensitive to poor initial guesses for $\theta(0)$ and $C_{1}$.

Numerical solutions of Equations (29) subject to the boundary conditions given by Equations (30) were obtained for Rayleigh numbers ranging from $10^{-3}$ to $10^{2}$. For comparative purposes, the results for Rayleigh numbers of $0.1,1.0$, 10, and 100 are displayed in the accompanying figures. A computer code, SHOOT2, 7 based on a shooting procedure which uses current state-of-the-art variable step size integration methods was employed for the solution of the boundary value problem. The integration was carried out to a value of 1000 for $n$ in all cases. This interval was found to be of sufficient magnitude to ensure the accuracy of solutions in regions of physical interest.

Numerically determined values for $\theta(\eta), f(\eta)$, and $f^{\prime}(\eta)$ are plotted versus $n$, with Rayleigh number as a parameter, in Figures 1 through 3. For convenience. in plotting, it was necessary to scale the various functions. Numerical values of the scaling parameters $\theta(0)$ and $f(\infty)$ are tabulated in Table 1 . In Figures 1 through 3, the dashed curves are obtained from the large Rayleigh number solutions given by Equations (22) and (23). Comparisons are made only for Rayleigh numbers of 10 and 100. For a Rayleigh number of 1.0, the large Rayleigh number approximation is no longer valid, hence no comparison to the numerical results is attempted. The small Rayleigh number approximations, as given by Equations (20) and (21), are indistinguishable from the curves for a Rayleigh number of 0.1 as plotted in Figures 1 through 3 .

TABLE 1. Selected Numerical Results for the Semi-Infinite Region. Tabulated Values are Accurate to Four Significant Figures.

\begin{tabular}{rccccc}
$\mathrm{Ra}$ & $\theta(0)$ & $-\mathrm{C}_{1}, f(\infty)$ & $\mathrm{fl}^{\prime \prime}(0)$ \\
\hline 0.1 & $1.0106 \mathrm{E}-1$ & $5.0174 \mathrm{E}-2$ & 5.0889 & $\mathrm{E}-2$ \\
0.5 & 5.2461 & $\mathrm{E}-1$ & $2.5274 \mathrm{E}-1$ & 2.7186 & $\mathrm{E}-1$ \\
1 & 1.0959 & $\mathrm{I}+0$ & $5.0673 \mathrm{E}-1$ & 5.8917 & $\mathrm{E}-1$ \\
5 & $6.0983 \mathrm{E}+0$ & $1.9746 \mathrm{E}+0$ & 4.1237 & $\mathrm{E}+0$ \\
10 & $1.1213 \mathrm{E}+1$ & 2.6640 & $\mathrm{E}+0$ & 8.5489 & $\mathrm{E}+0$ \\
50 & $4.2955 \mathrm{E}+1$ & $3.6091 \mathrm{E}+0$ & 3.9346 & $\mathrm{E}+1$ \\
100 & $8.0541 \mathrm{E}+1$ & $3.7921 \mathrm{E}+0$ & $7.6749 \mathrm{E}+1$
\end{tabular}




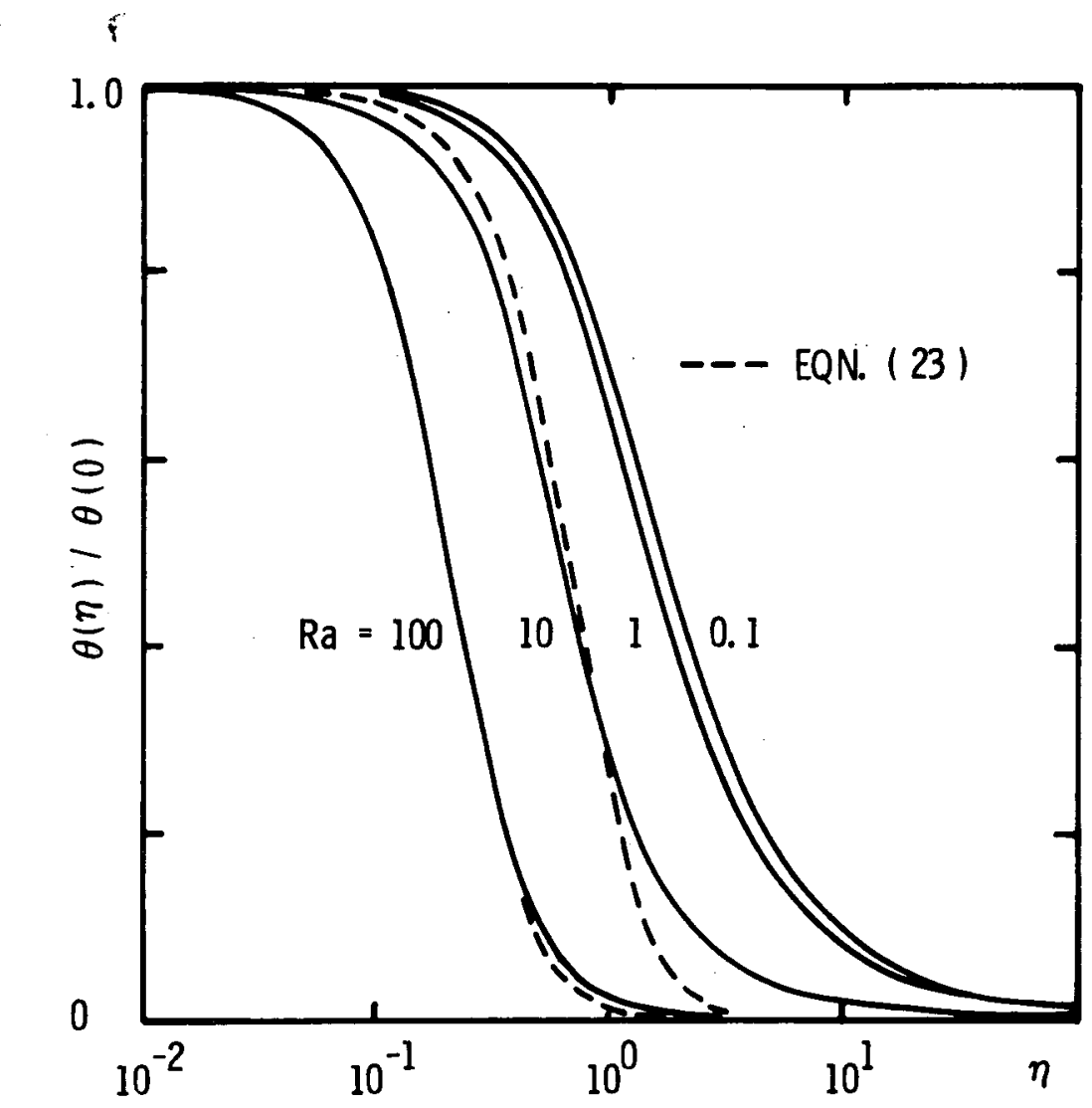

FIGURE 1 .

The Function $\theta(n) / \theta(0)$ for the Semi-Infinite Region.

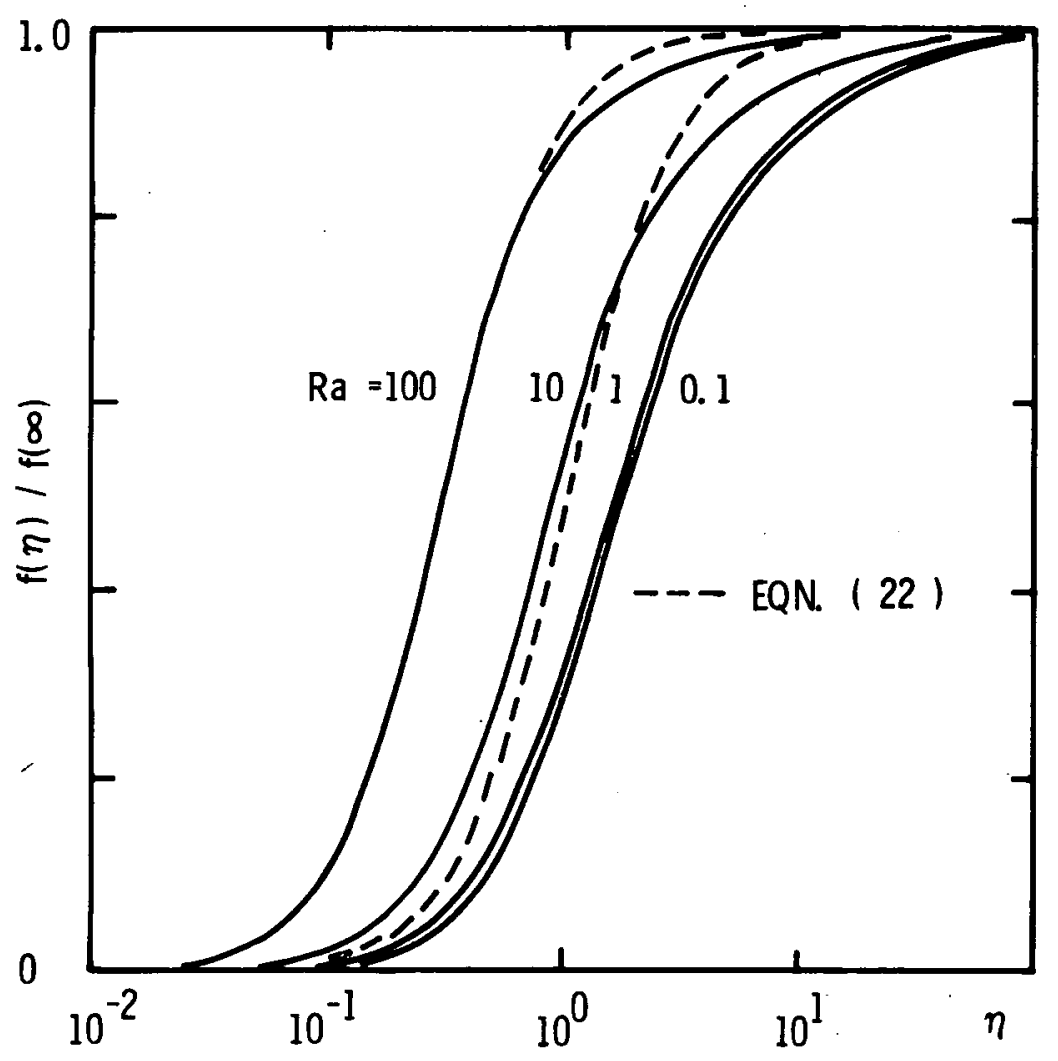

FIGURE 2 .

The Function $f(n) / f(\infty)$ for the Semi-Infinite Region. 


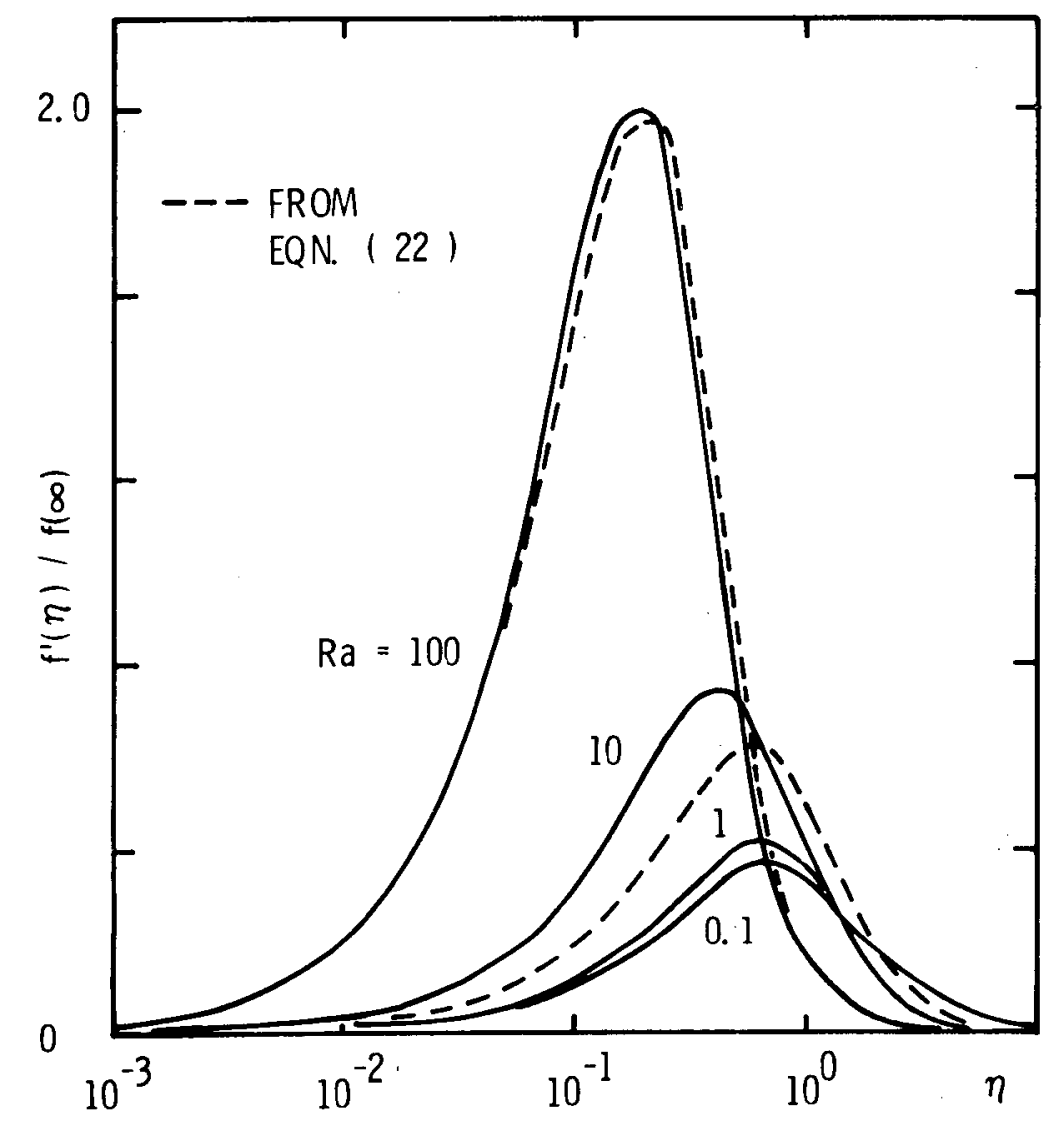

FIGURE 3 .

The Function $f^{\prime}(\eta) / f(\infty)$ for the Semi-Infinite Region.

If the constant of integration $C_{1}$ and the function $\theta(0)$ are known a priori for a specified Rayleigh number, then Equations (29) and (30) can be integrated in a straightforward manner. As a guide to the selection of $C_{1}$ and $\theta(0)$, values for these parameters are tabulated for selected Rayleigh numbers in Table 1. Since $C_{1}=-f(\infty)$, the approximate analytical solutions given by Equations (20) and (22) can be used to show that the limiting values for $C_{1}$ are $-R a / 2$ for small Rayleigh number and -4 for large Rayleigh number. Limiting values for $\theta(0)$, as obtained from Equations (21) and (23), are Ra for small Rayleigh number and $3 \mathrm{Ra} / 4$ for large Rayleigh number.

\section{Point Source in an Infinite Medium}

The numerical solution procedure utilized for this problem closely parallels that of the preceding case. The basic differential equations (15) and (16) can be integrated once to provide 


$$
\begin{gathered}
f^{\prime}=-\eta \theta+c_{2}, \\
f \theta=\left(1-n^{2}\right) \theta^{\prime},
\end{gathered}
$$

where $\mathrm{C}_{2}$ is a constant of integration, and the constant of integration associated with Equation (32) is zero by virtue of the boundary conditions given by Equations (19). As before, the integral constraint given by Equation (17) is rewritten as

$$
s(\eta)=\int_{-1}^{n}\left(1-f^{\prime}\right) \theta d \zeta .
$$

Now the original boundary value problem can be expressed by the system

$$
\begin{aligned}
& \theta^{\prime}(\eta)=\left(1-\eta^{2}\right)^{-1} f \theta, \\
& f^{\prime}(\eta)=-n \theta+c_{2}, \\
& c_{2}^{\prime}(\eta)=0, \\
& S^{\prime}(\eta)=\left(1+\eta \theta-c_{2}\right) \theta,
\end{aligned}
$$

subject to the boundary conditions

$$
\begin{gathered}
f(-1)=0=f(1), \\
S(-1)=0, S(1)=R a .
\end{gathered}
$$

A complication arises due to the singular nature of Equation (34a) at the boundary points. This difficulty is, however, easily removed since a straightforward analysis using Equations $(34 \mathrm{a})$ and $(34 \mathrm{~b})$ shows that $\theta^{\prime}( \pm 1)=\mp\left(\frac{1}{2}\right) \theta( \pm 1)$ $f^{\prime}( \pm 1)$. The numerical solution of the differential system given by Equations (34) subject to the boundary conditions specified by Equations (35) was obtained through use of the computer code SUPOR $Q^{8}$ which utilizes quasi-linearization techniques coupled with superposition and an orthonormalization process.

Numerically determined values for $\theta(\eta), f(n)$, and $f^{\prime}(\eta)$ are plotted versus the angle $\Phi$, with Rayleigh number as a parameter, in Figures 4 through 6 . Values for the scaling parameters used in these figures are tabulated in Table 2. 
TABLE 2. Selected Numerical Results for the Infinite Region.

Tabulated Values are Accurate to Four Significant Figures.

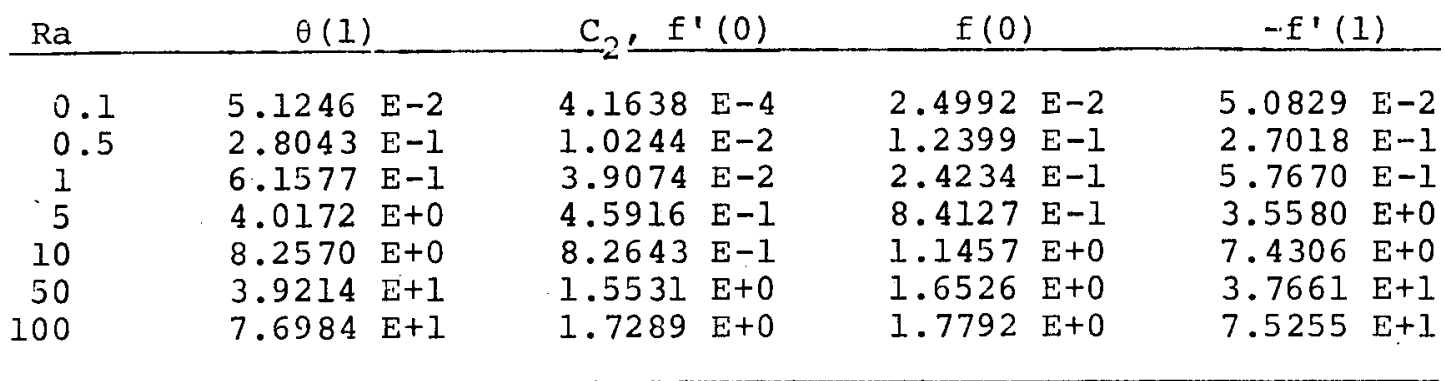

The dashed curve in Figure 4 represents the low Rayleigh number solution given by Equation (25). For a Rayleigh number of 0.1 , the approximate solution is indistinguishable from the numerical results when plotted in Figure 4 . The approximate results given by Equation (24) and its derivative are essentially identical to the numerical results for Rayleigh numbers of 0.1 and 1.0 when plotted in Figures 5 and 6 . For a Rayleigh number of 10, the small Rayleigh number approximation is no longer valid, hence no further comparison is attempted.

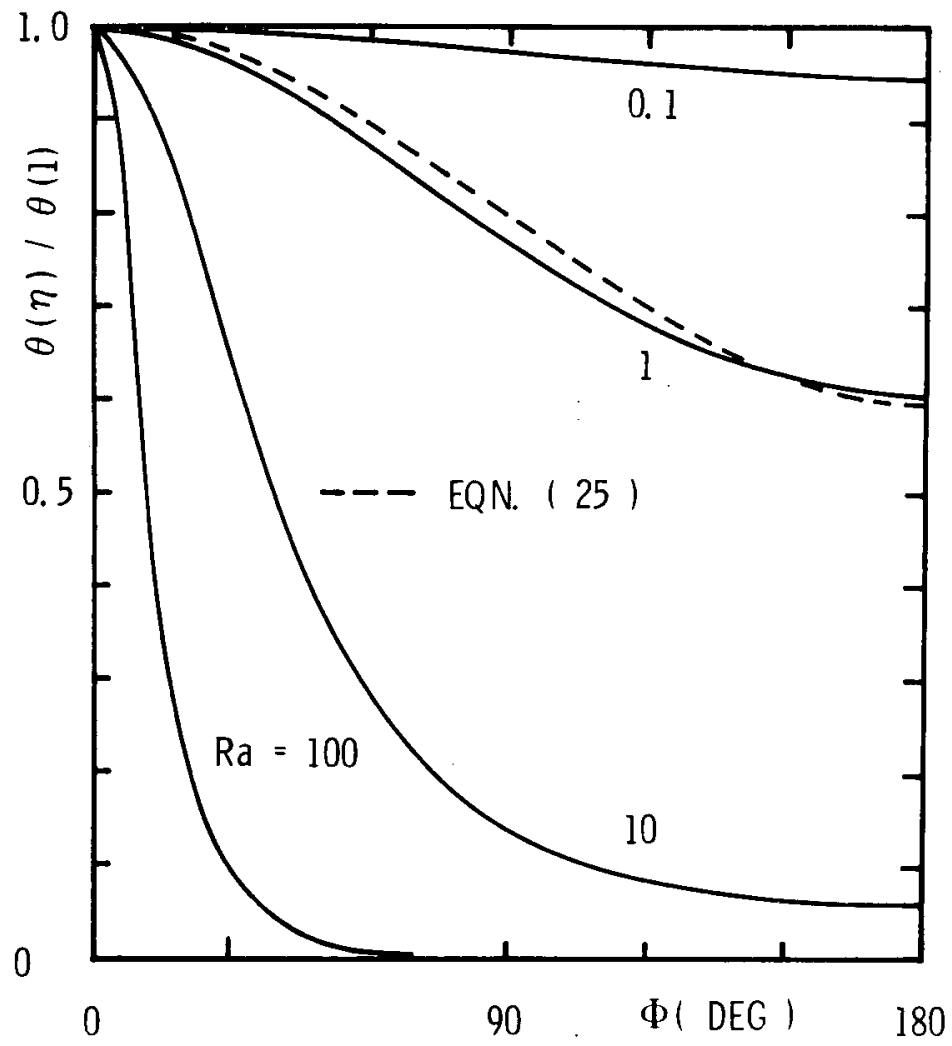

FIGURE 4. The Function $\theta(n) / \theta(1)$ for the Infinite Region. 


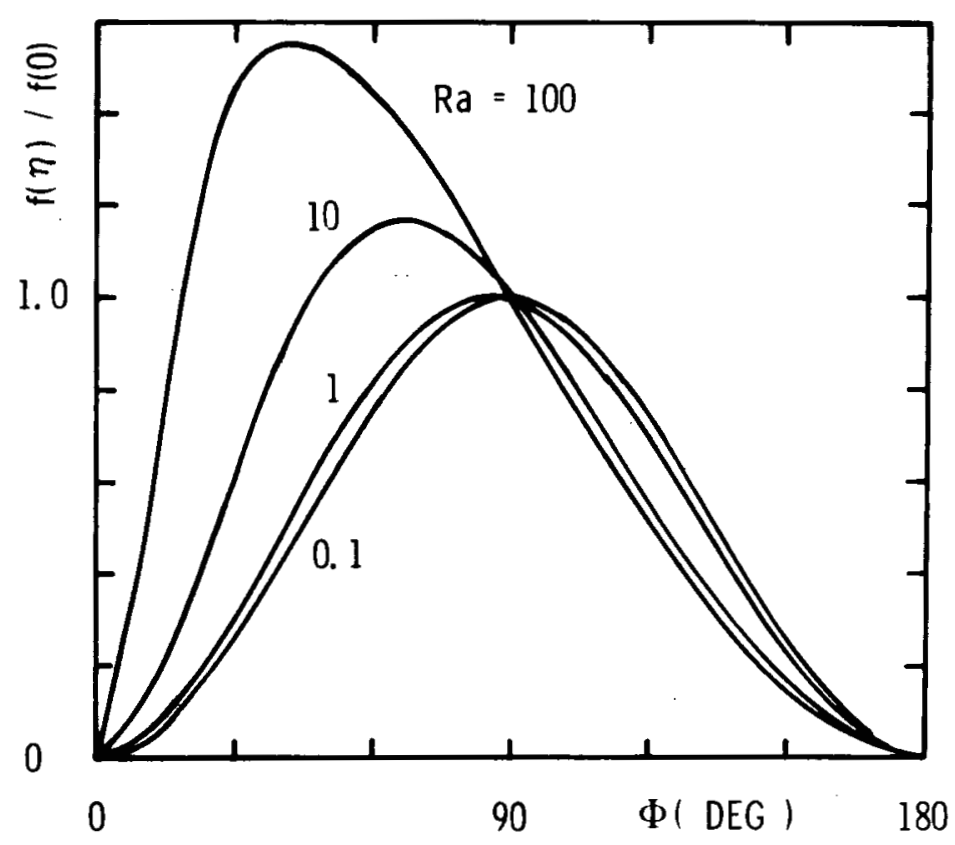

FIGURE 5. The Function $f(\eta) / f(0)$ for the Infinite Region.

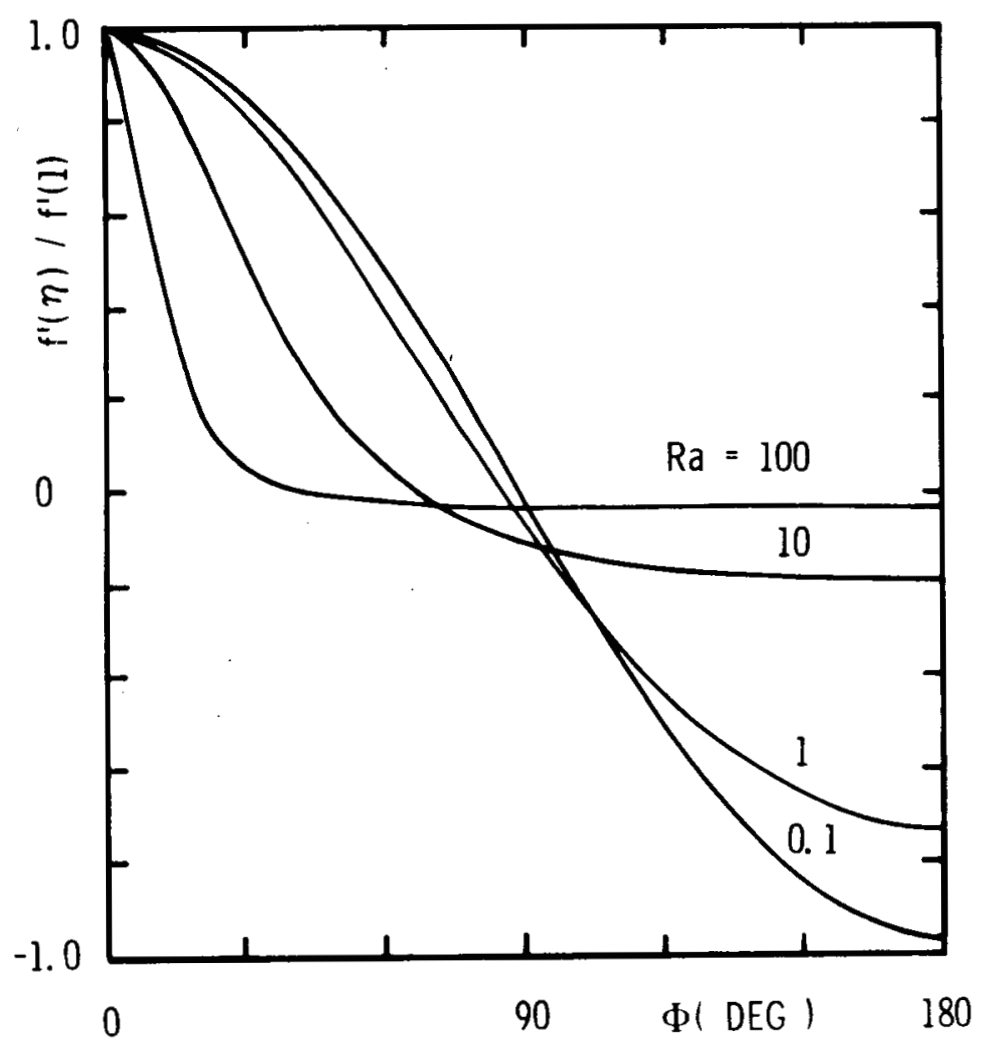

FIGURE 6. The Function $f^{\prime}(n) / f^{\prime}(1)$ for the Infinite Region. 
A knowledge of the values of $\mathrm{C}_{2}$ and $\theta(1)$ for a specified Rayleigh number will allow Equations (34) and (35) to be integrated in a straightforward manner. Numerical values for these parameters are tabulated for selected Rayleigh numbers in Table 2 . Noting that $\mathrm{C}_{2}=\mathrm{f}^{\prime}(0)$, the approximate solution given by Equation (24) can be used to show that the limiting value for $\mathrm{C}_{2}$ for small Rayleigh number is $\mathrm{Ra}^{2} / 24$. Numerical results have been used to establish a limit of approximately 2 for large Rayleigh number. For small Rayleigh number, Equation (25) yields a limiting value of $\mathrm{Ra} / 2$ for $\theta(1)$, and numerical results indicate a value of approximately $3 \mathrm{Ra} / 4$ for large Rayleigh number.

\section{Vertical Transport of Fluid Particles}

A simple expression can be derived for the vertical transport of a fluid particle which is initially coincident with the point source. From a consideration of Equations (11) and (18), it can be shown that the velocity along the axis of symmetry, for both cases considered, is

$$
w=\alpha F / z,
$$

where the parameter $F$ is given by $f^{\prime \prime}(0)$ for the semi-infinite region and $-f^{\prime}(1)$ for the infinite region. These parameters are tabulated for selected values of the Rayleigh number in Tables 1 and 2. If it is assumed that the fluid particle is initially coincident with the point source, then the total vertical transport $\mathrm{H}$ in a time interval $t$ is expressed by

$$
\mathrm{H}=(2 \alpha \mathrm{Ft})^{1 / 2} \text {. }
$$

Limiting values for f"(0) for the semi-infinite region, as established from Equations (20) and (22), are $\mathrm{Ra} / 2$ for small Rayleigh number and 3Ra/4 for large Rayleigh number. For the infinite region, Equation (24) yields a limiting value of $\mathrm{Ra} / 2$ for the function $-f^{\prime}(1)$ for small Rayleigh number, and numerical results yield an approximate limiting value of 3Ra/4 for large Rayleigh number. 
Accurate numerical solutions for the steady, axisymmetric velocity and temperature fields associated with a point source of thermal energy in a fluidsaturated porous medium were obtained through use of similarity transformations. The two cases considered were those of a point source located on the lower, insulated boundary of a semi-infinite region and a point source embedded in an infinite region. Representative plots of the isotherms and streamlines for each case considered are presented in Figures 7 and 8 for a Rayleigh number of 10 .

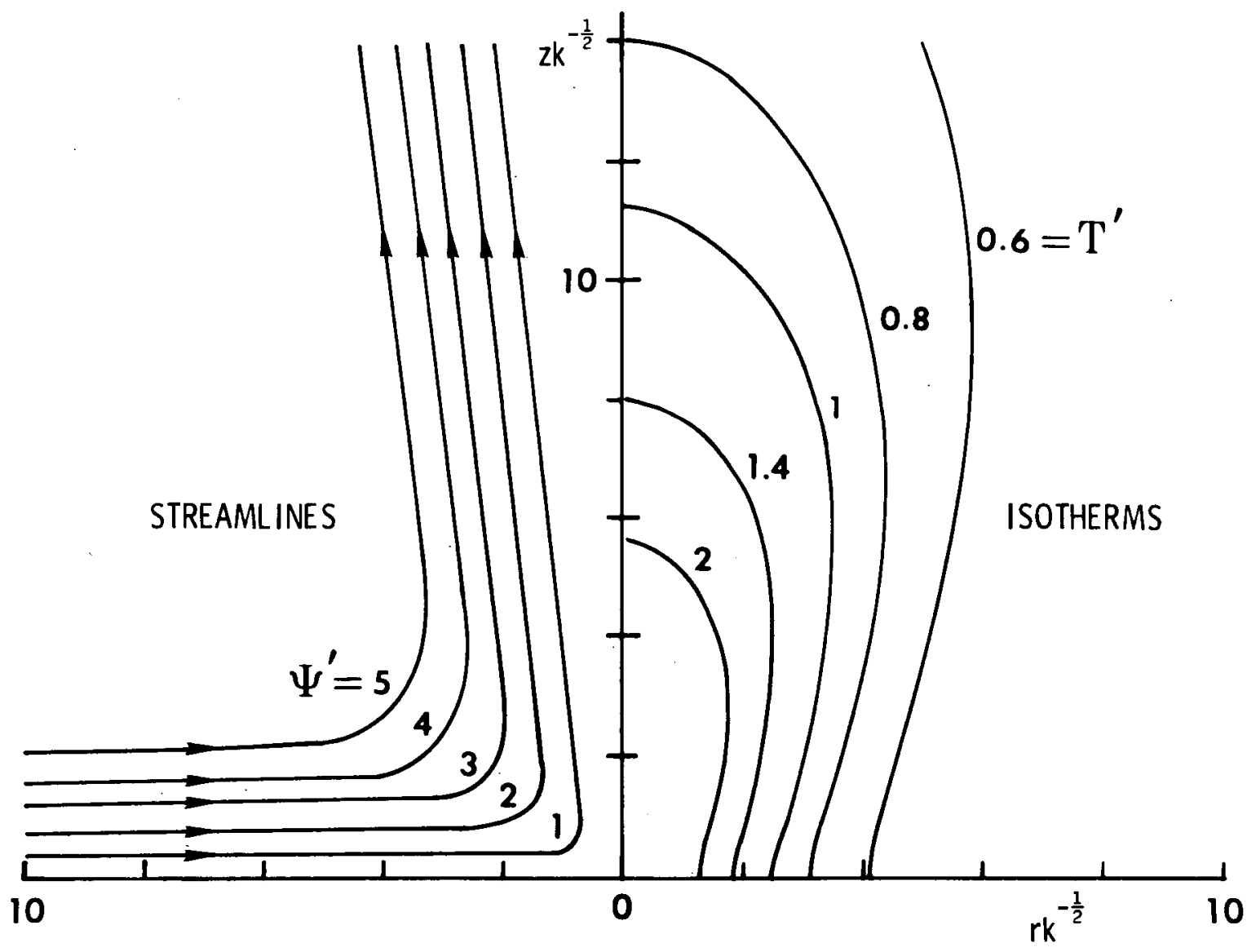

FIGURE 7. Streamlines and Isotherms for a Point Source at the Base of a SemiInfinite Region, $\mathrm{Ra}=10, \psi^{\prime}=\psi / \alpha \mathrm{k}^{1 / 2}, \mathrm{~T}^{\prime}=\left(\mathrm{T}-\mathrm{T}_{\infty}\right) \rho_{\infty} g \beta \mathrm{k}^{3 / 2} / \mu \alpha$. 


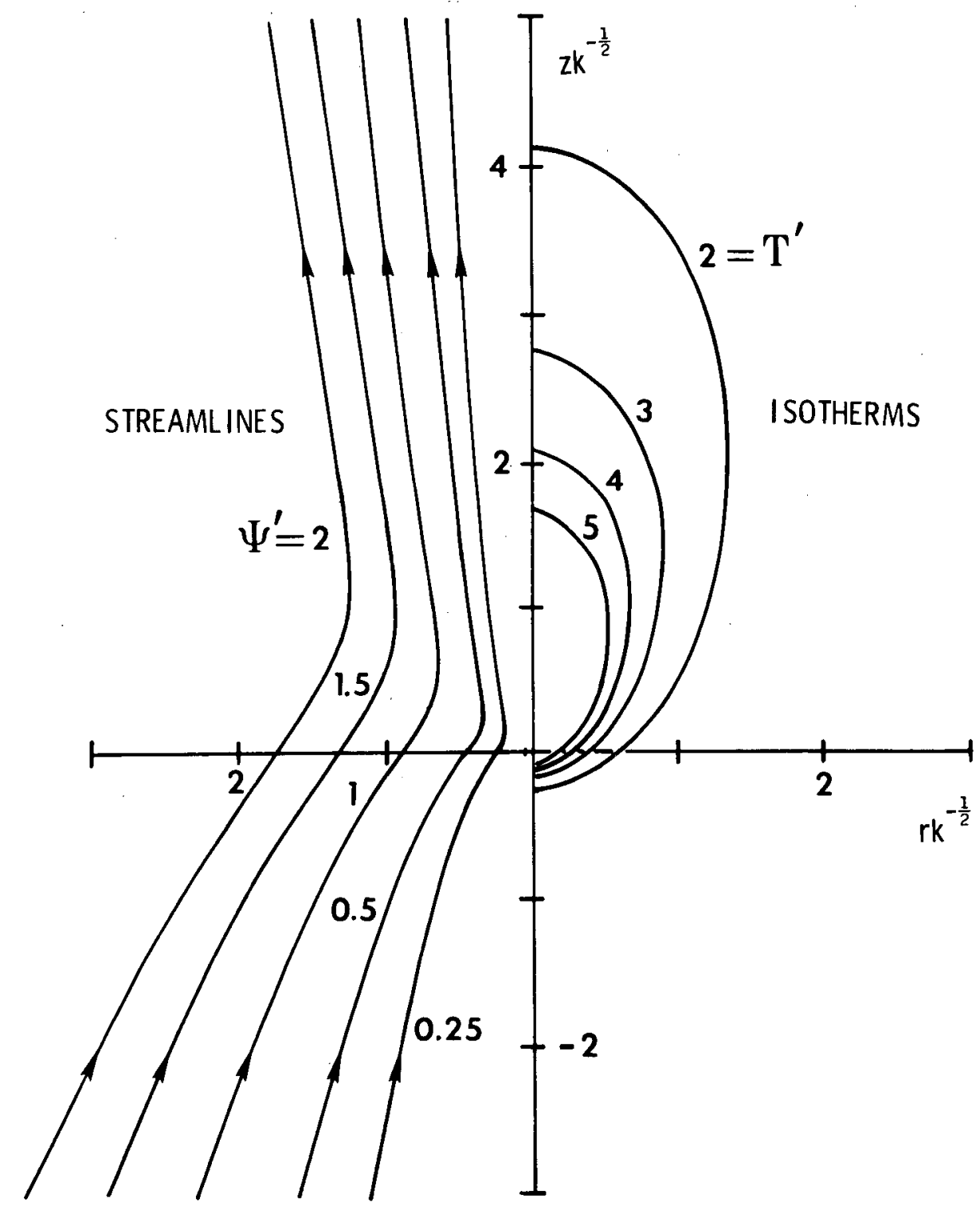

FIGURE 8. Streamlines and Isotherms for a Point Source in an Infinite Region; $\mathrm{Ra}=10, \psi^{\prime}$ and $T^{\prime}$ Defined in Figure 7 . 
A careful examination of the numerical results indicates that, for a Rayleigh number of 0.1 , the temperature distribution is closely approximated by the steady-state conduction solution. Isothermal surfaces are approximately hemispherical or spherical and are centered about the point source. The results for low Rayleigh number given by Equations $(20),(21),(24)$, and (25) then closely approximate the numerical solutions. When the Rayleigh number is 100 , the solutions exhibit a plume-like behavior in agreement with the boundary layer model proposed by wooding. For the semi-infinite region, the results for large Rayleigh number given by Equations (22) and (23) are found to be reasonably accurate, even for a relatively modest value of 100 for the Rayleigh number. In general, the numerical results exhibit a smooth transition from flows which are conduction dominated at low Rayleigh number to flows with boundary layer behavior at large Rayleigh number.

The plots of the numerical results presented provide reasonably accurate estimates for the important features of the velocity and temperature fields. If, however, more detailed results are needed, tabulated numerical values for all important parameters are contained in the appendices for an extended range of Rayleigh numbers. 
APPENDIX A

TABULATED NUMERICAL RESULTS FOR A POINT SOURCE AT THE BASE OF A SEMI-INFINITE REGION

The numerical results tabulated in this appendix are solutions to Equations (28), (29), and (30). Reading from left to right, the parameters tabulated are: $\eta, \theta(n), \theta^{\prime}(n), f(n), f^{\prime}(\eta), f^{\prime \prime}(\eta), S(n), z u(\eta) / \alpha, z w(n) / \alpha$, and $\left(z^{2} / \alpha\right) \frac{\partial w}{\partial r}$. The parameter $S(n)$ is defined in Equation $(28)$. When $n$ is large, $S(n)$ becomes equal to the Rayleigh number. Hence, the value of $S(\eta)$ for $\eta=1000$ corresponds to the Rayleigh number for the solution. Results are tabulated for Rayleigh numbers of $0.001,0.01,0.1,0.5,1.0,5.0,10,50$, and 100 . 


\begin{tabular}{|c|c|c|c|}
\hline & & THE IAP & \\
\hline 0.0000 & $1.0011 E-03$ & 0. & 0. \\
\hline .0100 & $1.0010 E-03$ & $-1.0012 E-05$ & $2.5027 \Xi-08$ \\
\hline .0200 & $1.0009 E-03$ & $-2.0015 E-05$ & $1.0009 E-07$ \\
\hline .0300 & $1.0006 E-03$ & $-3.0000 E-05$ & $2.2511=-07$ \\
\hline .0400 & $1.0003 E-03$ & $-3 \cdot 9958 E-05$ & $3.9999 E-07$ \\
\hline .0500 & $9.9985 E-04$ & $-4.9880 E-05$ & $6.2456 E-37$ \\
\hline .0600 & $9.9930 E-04$ & $-5.9758 E-05$ & $8.9363 E-07$ \\
\hline .0700 & $9.9865 E-04$ & $-6.9582 E-05$ & $1.2219 \equiv-06$ \\
\hline .0800 & $9.9791 E-04$ & $-7.9345 E-05$ & $1.5942 \Xi-06$ \\
\hline .0900 & $9.9707 E-04$ & $-8.9037 E-05$ & $2.0151 E-06$ \\
\hline .1000 & $9.9613 E-04$ & $-9.8651 E-05$ & $2.4843 E-06$ \\
\hline .2000 & $9.8165 E-04$ & $-1.8883 E-04$ & $9.7210 E-06$ \\
\hline .3000 & $9.5887 E-04$ & $-2.6397 E-04$ & $2.1111 \equiv-05$ \\
\hline .4000 & $9.2948 E-04$ & $-3.2058 E-04$ & $3.5803 E-05$ \\
\hline .5000 & $8.9539 E-04$ & $-3.5823 E-04$ & $5.2847 \Xi-05$ \\
\hline .6000 & $8.5840 E-04$ & $-3.7878 E-04$ & $7.1335 E-05$ \\
\hline .7000 & $8.2010 E-04$ & $-3.8535 E-04$ & $9.0487 \equiv-05$ \\
\hline .8000 & $7.8169 E-04$ & $-3.8138 E-04$ & $1.0969 E-04$ \\
\hline .9000 & $7.4407 E-64$ & $-3.7004 E-04$ & $1.2850 \equiv-04$ \\
\hline 1.0000 & $7.0783 E-04$ & $-3.5397 E-04$ & $1.4561 E-04$ \\
\hline 2.0000 & $4.4765 E-04$ & $-1.7907 E-04$ & $2.7669 E-04$ \\
\hline 3.0000 & $3.1653 E-04$ & $-9.4963 E-05$ & $3.4225 E-04$ \\
\hline 4.0000 & $2.4277 E-04$ & $-5.7123 E-05$ & $3.7913 \equiv-04$ \\
\hline 5.0000 & $1.9630 \varepsilon-04$ & $-3.7751 E-05$ & $4.0236 \Xi-04$ \\
\hline 6.0000 & $1.6+56 E-04$ & $-2.6685 E-05$ & $4.1824 E-04$ \\
\hline 7.0000 & $1.4156 E-04$ & $-1.9818 E-05$ & $4.2974 \Xi-04$ \\
\hline 8.0000 & $1.2415 E-04$ & $-1.5280 E-05$ & $4.3844 \geqq-04$ \\
\hline 9.0000 & $1.1054 E-04$ & $-1.2132 E-05$ & $4.4525 E-04$ \\
\hline 10.0000 & $9.9598 E-05$ & $-9.8612 E-06$ & $4.5072 E-04$ \\
\hline 20.0000 & $4.9985 E-05$ & $-2.4930 E-06$ & $4.7553 E-04$ \\
\hline 30.0000 & $3.3346 E-05$ & $-1.1103 E-06$ & $4.8385=-04$ \\
\hline 40.0000 & $2.5016 E-05$ & $-6.2500 E-07$ & $4.8803 E-04$ \\
\hline 50.0000 & $2.0015 E-05$ & $-4.0014 E-07$ & $4.9053 E-34$ \\
\hline 60.0000 & $1.6680 E-05$ & $-2.7792 E-07$ & $4.9220 E-04$ \\
\hline 70.0000 & $1.4278 E-05$ & $-2.0421 E-07$ & $4.3340 E-04$ \\
\hline 80.0000 & $1.2511 E-05$ & $-1.5636 E-07$ & $4.9430 \equiv-04$ \\
\hline 90.0000 & $1.1121 E-$ & $-1.2355 E-07$ & $4.7500 E-04$ \\
\hline .0000 & $1.0009 E-05$ & $-1.0008 E-07$ & $4.9556 E-04$ \\
\hline 000000 & $5.0047 E-06$ & $-2.5023 E-08$ & $4.9811 E-04$ \\
\hline 300.0000 & $3.3365 E-06$ & $-1.1121 E-$ & $4.9899 E-04$ \\
\hline .0000 & $2.5024 E-06$ & $-6.2558 E-09$ & $4.9946 \Xi-04$ \\
\hline .0000 & $2.0019 E-06$ & $-4.0337 E-09$ & $4.3976 E-04$ \\
\hline 00.0000 & $1.6682 E-06$ & $-2.7804 E-09$ & $4.9998 \equiv-04$ \\
\hline & $1.4299 E-06$ & $-2.0427 E-09$ & $5.0015 E-04$ \\
\hline 300.0000 & $1.2512 E-00$ & $-1.5640 E-09$ & $5.0029 E-04$ \\
\hline 000.0000 & $1.1122 E-06$ & $-1.2357 E-09$ & $5.0041 E-04$ \\
\hline 1000.0 & $1.0009 E-06$ & $-1.0009 E-09$ & $5.0051 E-04$ \\
\hline
\end{tabular}

$5.0051 E-06$ . $0005 E-05$ $1.4997 E-05$ $1.9975 E-05$ $2.9874 E-05$ $3.4785 E-05$ $3.9665 \bar{E}-05$ $4.4511 E-05$ $4.9317 E-05$ $9.4396 E-05$ $1.3196 E-C 4$ $1.6027 E-04$ $1.7909 E-04$ $1.8936 E-04$ $1.9265 E-04$
$1.9066 E-04$ $1.9066 E-04$
$1.8500 E-04$ $1.7697 E-04$ $8.9533 E-05$ $4.7481 E-05$ 1.8562E-05 1.8345E-05 $9.9094 E-06$ $96406 E-06$ $6.0665 E-06$ $4.06311=-06$ $1.2470 E-06$ $5.5565 E-07$ $3.1300 E-07$ $2.0057 E-07$ $1.3946 E-07$ $1.0261 E-07$ $7.8681 \mathrm{E}-08$ $5.2276 E-08$ $5.0540 E-08$ $1.3012 E-08$ $6.0612 E-09$ $3.6284 E-09$ $2.5023 E-09$ $1.8907 \Sigma-09$ $1.5218 E-09$ $1.2825 E-09$ $1.1183 E-09$
$1.0009 E-09$
$F P P$

$5.0058 E-04$ $5.0036 E-04$
$4.9968 E-04$ $4.9968 E-104$
$4.9856 E-04$ $4.9856 E-04$
$4.9699 E-04$ $4.9498 E-04$ $4.9253 E-04$ 4.8956E-04 $4.8636 E-04$ $4.8264 E-04$ $4.7852 E-04$ $4.1751 E-04$ $3.3090 E-04$ $2.3485 E-04$ $1.4325 E-04$ $6.4954 E-05$ $3.6702 E-06$ $-4.0713 E-05$ $-7.0431 E-05$ $-8.8502 E-05$ $-6.2676 E-05$ $-2.6906 E-05$ $-7.1146 E-05$ -4.2672E-0 $-2.7462 E-05$ $-1.8660 E-06$ $-1.3233 E-05$ $-9.7148 \mathrm{E}-07$ $-1.2418 \mathrm{E}-07$ $-3.6949 E-08$ $-1.5610 E-08$ $-7.9979 E-09$ $-4.6301 E-09$ $-2 \cdot 9164 E-09$ $-1.9541 E-09$ $-1.3725 E-09$ $-1.0006 \mathrm{E}-09$ $-1.2511 E-10$ $-3.7071 E-11$ $-1.5639 \mathrm{E}-11$ $-8.0074 E-12$ $-4.5340 E-12$ $-2.3182 E-12$ $-1.9550 E-12$ $-1.3730 E-12$
$-1.0009 E-12$
0. $2.0026 E-07$ $4.5042 E-07$ $8.0032 \varepsilon-07$ $.2497 E-06$
$1.7980 E-06$ $2.4449 E-06$ $3.1898 E-06$ $4.0320 E-06$ $4.9707 E-06$ $1.9450 \mathrm{E}-05$ $4.2240 E-05$
$7.1634 E-05$ $1.0573 E-04$ $1.4272 \mathrm{E}-04$ $1.8104 E-04$ $2.1945 E-04$ 2.5708 E-0 4 $2.9331 E-04$ $5.5348 E-04$ ..8458E-0 7.5833E 04 8. $3651 E-04$ $8.5951 E-04$ $8.7690 E-04$ $8.9651 E-04$ 9.0145E-04 $9.0145 E-04$
$9.5104 E-04$ $9.6767 E-04$ $9.67600 E-04$ 9.8100E-04 $9.8433=-04$ $9.8671 E-04$ $9.8850 E-04$ $9.8989 E-04$ $9.3100 E-04$ $9.9600 E-04$ 9.9767E-04 $9.9850 E-04$ $909900 E-04$ $9.9933 E-04$ $9.9957 E-04$ $9.9975 E-04$ $9.9989 E-04$
$1.0000 E-03$
Z/A U

$2.5024 E-06$ 5.0013E-06 $7.4936 \mathrm{E}-06$ $9.9757 E-06$ $1.4896 E-05$ $1.7329 E-05$ $1.973 \mathrm{BE}-05$ $2.2120 E-05$ $4.5791 E-05$ E.1592E-05 7.075 \&E-0 05 $7.3394 E-05$ $7.0473 E-05$ $6.3383 E-05$ $5.3552 E-05$ $4.2222 E-05$ उ.0356E 05 $-6.66025-05$ $-6.6221 E-05$ $-5.6364 E-05$ $-5.1482 E-05$ $-4.7164 E-05$ $-4.3406 E-05$ $-4.0141 E-05$ $-2.253 C E-05$ $-1.5573 E-05$ $-1.1888 \mathrm{EE}-05$ $-9.610 \mathrm{CE}-06$ -8.063 SE -06 . $-6.1000 E-06$ $-4.9050 E-06$ -2.477 SE-O $-1.6573 E-06$ $-1.2450 E-06$ $-9.9702 E-07$ $-8.3141 E-07$ $-7.1298 E-0$ $-6.2408 E-07$ -5.548 SE -07
$-4.9951 E-07$
Z/A H

$5.0058 E-04$
$5.0051 E-04$

$5.0051 E-04$
$5.0028 E-04$

$5.0028 E-04$

$4.9939 E-04$

$4.9871 E-04$

4. $9789 E-04$

$4.9693 E-04$

$.9582 E-04$

..9456E-04

$4.9317 E-04$

$4.7198 E-04$

$4.0066 \mathrm{E}-04$

$3.5818 E-0$

$3.1561 E-04$

$2.7521 E-04$

$2.3833 E-04$

$1.765 E-04$

$1.7697 E-04$

$1.5827 E-05$

7.1404E-06

$7.1404 E-06$
$3.7752 E-06$

$6.7406 E-07$

$4.9311 E-07$

$6.2350 \mathrm{E}-08$

$1.8522 E-08$

$7.8251 E-09$

$2.3244 E-09$

$1.4658 E-09$

9.8351E-10

6.9195E-10

6.0540E-10

$2.0204 E-11$

$9.0710 \mathrm{E}-1$

5.0047E-12

$3.1511 \mathrm{E}-12$

$2.1741 \mathrm{E}-12$

$1.6031 E-12$
$1.2426 E-12$

$1.0009 E-12$
$2 / A$ DHDR

$-1.5016 E-05$ $-1.5016 E-05$ $-4.4957 E-05$ $-5.9838 E-05$ $7.4629 E-05$ $-8.9310 E-05$ $-1.0386 E-04$ $1.1825 E-04$ $-2.7233 E-04$ $-3.6324 E-04$ $-4.1452 E-04$ $-4 \cdot 2985 E-04$ $-3.8792 E-04$ $-3.4880 E-04$ - . $0665 E-04$ $-5.3721 E-05$ $-1.4244 E-05$ $-5.0403 E-05$ . $1780 E-06$ $-1.0818 E-06$ $-5.9455 E-07$ $-3.5263 E-07$ $-1.4646 E-C 7$ $-1.8490 E-0$ $-5.8589 E-10$ $-2.4019 E-10$ $-1.1591 E-10$ $-6 \cdot 2603 E-11$ $-3.6720 \mathrm{E}-1$ $-2.2939 \mathrm{E}-11$ $-1.5060 E-11$ $-9.5084 E-13$ $-1.9092 E-13$ $-4.2746 E-15$ $-4.4475 E-15$ $-2.0019 E-15$ 



\begin{tabular}{|c|c|c|c|c|c|}
\hline$x$ & THETA & THETAP & $\mathrm{F}$ & $F P$ & FPP \\
\hline 0.0000 & $1.0020 E-02$ & 0 & 0. & 0 & $5.0133 E-03$ \\
\hline .0100 & $1.0019 E-02$ & $-1.0043 E-04$ & $2.5065 E-07$ & $5.0126 E-05$ & $5.0111 E-03$ \\
\hline .0200 & $1.0018 E-02$ & $-2.00077 E-04$ & $1.0024 \equiv-06$ & $1.0021 E-04$ & $5.0043 E-03$ \\
\hline .0300 & $1.0015 E-02$ & $-3.0094 E-04$ & $2.2545 E-06$ & $1.5020 E-04$ & $4.9930 E-03$ \\
\hline $.040 \mathrm{C}$ & $1.0012 E-02$ & $-4.0083 E-04$ & $4.0059 E-06$ & $2.0005 E-04$ & $4.9773 E-03$ \\
\hline .0500 & $1.0007 E-02$ & $-5.0036 E-04$ & $6.2549 \Xi-06$ & $2.4973 E-04$ & $4.9572 E-03$ \\
\hline .0600 & $1.0002 E-02$ & $-5 \cdot 9944 E-04$ & $8.9997 E-66$ & $2.9918 E-04$ & $4.9326 E-03$ \\
\hline .0700 & $9.9951 E-03$ & $-6.9799 E-04$ & $1.2238 E-05$ & $3.4837 E-04$ & $4.9038 E-03$ \\
\hline & $9.9876 E-03$ & $-7.7591 E-04$ & $1.5966 E-05$ & $3.9724 E-04$ & $4.8707 E-03$ \\
\hline .0900 & $9.9792 E-03$ & $-8.9313 E-04$ & $2.0181 \equiv-05$ & $4.4577 E-04$ & $4.8334 E-03$ \\
\hline .1000 & $9.9698 E-03$ & $-9.8956 E-04$ & $2.4880 \equiv-05$ & $4.9390 E-04$ & $4.7921 \mathrm{E}-03$ \\
\hline .2000 & $9.8246 E-03$ & $-1.8939 E-03$ & $9.7352 E-05$ & $9.4532 E-04$ & $4.1806 E-03$ \\
\hline .3000 & $9.5961 \varepsilon-03$ & $-2.6473 E-03$ & $2.1142 E-04$ & $1.3214 \Xi-03$ & $3.3124 E-03$ \\
\hline .4000 & $9.3014 E-03$ & $-3.2146 E-03$ & $3.5852 E-04$ & $1.6047 E-03$ & $2.3499 E-03$ \\
\hline .5000 & $8.9595 E-03$ & $-3.5914 E-03$ & $5.2917 \equiv-04$ & $1.7930 E-03$ & $1.4322 E-03$ \\
\hline .6000 & $8.5888 E-03$ & $-3.7967 E-03$ & $7.1426 \equiv-04$ & $1.8956 E-03$ & $6.4807 E-04$ \\
\hline .7000 & $8.2049 E-03$ & $-3.8618 E-03$ & $9.0597 \equiv-04$ & $1.9283 E-03$ & $3.4557 E-05$ \\
\hline .8000 & $7.8200 E-03$ & $-3.8212 E-03$ & $1.0982 E-03$ & $1.9082 E-03$ & $-4.0955 E-04$ \\
\hline .9000 & $7.4431 E-03$ & $-3.7069 \bar{E}-03$ & $1.28645-03$ & $1.8513 E-03$ & $-7.0672 E-04$ \\
\hline 1.0000 & $7.0802 E-03$ & $-3.5453 E-03$ & $1.4676 E-03$ & $1.7708 E-03$ & $-8.8726 E-04$ \\
\hline 2.0000 & $4.4758 E-03$ & $-1.7915 E-03$ & $2.7688 \equiv-03$ & $8.9532 E-04$ & $-6.2709 E-04$ \\
\hline 3.0000 & $3.1643 E-03$ & $-9.4964 E-04$ & $3.4243 E-03$ & $4.7469 E-04$ & $-2.6907 E-04$ \\
\hline 4.0000 & $2.4267 E-03$ & $-5.7112 \bar{E}-04$ & $3.7930 E-03$ & $2.8552 E-04$ & $-1.3018 E-04$ \\
\hline 5.0000 & $2 E-03$ & $-3.7740 E-04$ & $4.0253 E-03$ & $1.8868 E-04$ & $-7.1125 E-05$ \\
\hline 6.0000 & $1.6448 E-03$ & $-2.6675 E-04$ & $4.1840=-03$ & $1.3337 E-04$ & $-4.2657 E-05$ \\
\hline 7.0000 & $1.4149 E-03$ & $-1.9810 E-04$ & $4.2989=-03$ & $9.9049 E-05$ & $-2.7451 E-0 J$ \\
\hline 8.0000 & $1.2409 E-03$ & $-1.5274 E-04$ & $4.3859 E-03$ & $7.6370 E-05$ & $-1.8552 E-05$ \\
\hline 9.0000 & $1.1048 E-03$ & $-1.2127 E-04$ & $4.4539 E-03$ & $6.0636 E-05$ & $-1.3228 E-05$ \\
\hline 10.0000 & $9.9548 E-04$ & $-9.8567 E-05$ & $4.5086 E-03$ & $4.9287 E-05$ & $-9.7103 E-06$ \\
\hline 20.0000 & 4.9959E-0 4 & $-2.4918 E-05$ & $4.7566=-03$ & $1.2464 E-05$ & $-1.2412 E-06$ \\
\hline 30.0000 & $3.3329 E-04$ & $-1.1097 E-05$ & $4.8398 E-03$ & $5.5537 E-06$ & $-3.6930 E-07$ \\
\hline 40.0000 & $2.5003 E-04$ & $-6 \cdot 2468 E-06$ & $4.8815 \equiv-03$ & $3.1284 E-06$ & $-1.5602 E-07$ \\
\hline & $2.0005 E-04$ & $-3.9993 E-06$ & $4.9065 \equiv-33$ & $2.0047 E-06$ & $-7.9938 E-08$ \\
\hline 60.0000 & $1.6671 \mathrm{E}-04$ & $-2.7778 E-06$ & $4.9232 E-03$ & & $-4.6277 E-08$ \\
\hline 70.0000 & $1.4290 E-04$ & $-2.0411 E-06$ & $4.93525-03$ & $1.0255 E-06$ & $-2.91495-08$ \\
\hline 80.0000 & $1.2504 E-04$ & $-1.5628 E-06$ & $4.9442 E-03$ & $7.8640 E-07$ & $-1.9530 E-08$ \\
\hline 90.0000 & $1.1115 E-04$ & $-1.2349 E-06$ & $4.7512 \Xi-03$ & $6.2243 E-07$ & $-1.3718 E-08$ \\
\hline 100.0000 & $1.0004 E-04$ & $-1.0003 E-06$ & $4.9568 E-03$ & $500514 E-07$ & $-1.0001 E-08$ \\
\hline & $5.0021 E-05$ & $-2.5010 E-07$ & $4.9823 E-03$ & $1.3005 E-07$ & $-1.2504 E-09$ \\
\hline 300.0000 & $3.3347 E-05$ & $-1.1116 E-07$ & $4.9911 E-03$ & $5.0580 E-08$ & $-3.7051 \mathrm{E}-10$ \\
\hline $400.000 \mathrm{C}$ & $2.5011 E-05$ & $-6.2526 E-08$ & $4 \cdot 9958 E-03$ & $3.6265 E-08$ & $-1.5631 E-10$ \\
\hline 500.0000 & $2.0008 E-05$ & $-4.0017 \varepsilon-08$ & $4.9988 E-03$ & $2.5010 E-08$ & $-8.0033 E-11$ \\
\hline 600.0000 & $1.6574 E-05$ & $-2.7789 E-08$ & $5.0010 E-03$ & 1. $8897 E-08$ & $-4.6316 E-11$ \\
\hline 700.0000 & $1.4292 E-05$ & $-2.0417 E-08$ & $5.0026 E-03$ & $1 \cdot 5210 E-08$ & $-2.9167 E-11$ \\
\hline 800.0000 & $1.2505 E-05$ & $-1.5632 E-08$ & $5.0040 E-03$ & $1.2818 E-08$ & $-1.9539 E-11$ \\
\hline 900.0000 & $1.1116 E-05$ & $-1.2351 E-08$ & $5.0052 E-03$ & $1.1178 E-08$ & $-1.3723 E-11$ \\
\hline 1000.0 & $1.0004 E-05$ & $-1.0004 E-08$ & $5.0063 E-03$ & $1.0004 E-08$ & $-1.0004 E-11$ \\
\hline
\end{tabular}

5

$5.0346 E-07$ $2.0134 E-06$ $4.5284 E-06$ $8.0462 E-06$ $1.2564 E-05$ (2.4580E-05 $3.2069 E-05$ $4.0536 E-05$ $4.0973 E-05$ $4.9973 E-05$ $1.9553 E-04$
$4.2457 E-04$ T.1991E-04 $1.0624 E=03$ $1.4338 E-03$ $1.8183 E-03$ $2.2036 E-03$ $2.5809 E-03$ $2.9441 \mathrm{E}-03$ $5.5477 E-03$ $6.8569 \varepsilon-03$ $7.5927 E-03$ $8.0558 E-03$ $8.3721 E-03$ $3.5012 E-03$ $8.7745 \mathrm{E}-03$ 8.9101E-03 $9.0190 \mathrm{E}-03$ $9.5127 E-03$ $9.5783 E-03$ $9.7611 E-03$ 9.8109E-0 $9.8441 E-03$ $9.8678 E-03$ $9894 E-03$ $9.9104 E-03$ $9.9602 E-03$ $9.9602 E-03$ $9.9851 E-03$ 9. $9900 E-03$ $9.99034 E-03$ 9.9957E-0 $9.9975 E-03$ $9.9989 E-03$
Z/A

0 . 2.5061E-05 5.008 \&E -05 9.9906E 05 . $1.2463 E-C 4$ $1.7354 E-04$ $1.7354 E-04$ $2.2153 E-04$ $2.4510 E-04$ $4.5855 E-04$ 6.585E -04 $7.0837 E-04$ $7.3462 \mathrm{E}-04$ $7.0521 E-04$ $6.3407 E-O$ $5.3552 E-04$ $4.2198 E-04$ $3.0312 E-04$ -4.890 SE -04 $-6.6675 E-04$ $-6.6274 E-04$ $-6.1637 E-04$ $-5.639 \in E-04$ $-5.1508 E-04$ $-4.7187 E-0$ $-4.3425 E-04$ $-4.0157 E-04$ $-2.2537 E-04$ - $1.5577 E-04$ - $-1.1891 E-04$ $-8.0650 E-05$ $-8.0660 E-05$ $-6.1016 E-05$ $-5.4391 E-05$ $-4.9063 E-05$ $-2.4781 E-05$ $-1.6576 E-05$ $-1.6576 E-05$ $-9.972 E E-O 6$ $-8.3160 E-06$ $-7.1314 E-06$ $-6.2422 E-06$ $-5.5502 E-06$ $-4.9963 E-06$
Z/ A W

$5.0126 E-03$ $5.0103 E-03$ $5.0066 E-03$ $4.9946 E-03$

$4.9864 E-03$

$4.9767 E-03$

$4.9655 \mathrm{E}-03$

$4.9530 E-03$

$4.9390 E-03$

$4.4048 E-03$

$4.0117 E-03$

$3.5859 E-03$

$3.1594 E-03$

$2.7547 E-03$

$2.0570 \mathrm{E}-03$

$1.7708 E-03$

$4.4766 E-04$

$1.5823 E-04$

$7.1379 E-05$

$3.7736 E-05$
$2.2228 E-05$

$1.4150 E-05$

$9.5463 E-06$

$6.7373 E-06$

4.9287E-06

$6.2318 E-07$

$1.8512 E-07$

$7.8210 \varepsilon-08$

$2.3232 \mathrm{E}-08$

2.3232E-08

$1.4650 E-08$

6. $150 E-09$

$5.0514 E-09$

$6.5025 E-10$

$2.0193 E-10$

S.0663E-11

$5.0021 E-11$

$3.1495 E-11$

$2.1729 E-11$

$1.6022 E-11$

$1.2420 E-11$
$1.0004 E-11$
22/A DHDR

$-1.5054 E-04$ $-3.0086 E-04$ $-5.9992 E-04$ T. $4821 E-04$ 9540E-04 $1.0413 E-03$ $-1.1856 E-03$ $-1.3281 E-03$ $-1.4688 E-03$ $-2.7301 E-03$ $-3.6411 E-03$ $-4.1545 E-03$ $-4.3075 E-03$ . $1855 E-03$ $-3.4935 E-03$ $-3.0708 E-03$ -2.6580E-03 $-5.3737 E-04$ $-1.4243 E-04$ $-5.0390 E-05$ $-2.1772 E-05$ $-1.0814 E-05$ $-5.943 C E-06$ $-2.2183 E-06$ $-1.8481 E-08$ $-1.1585 E-09$ $-6.2571 E-10$ $-3.670 C E-10$ $-2.2927 \mathrm{E}-10$ $-6.1744 E-13$ $-2.6011 E-13$ $-1.2968 E-13$ $-7.2709 E-14$ $-4.4452 E-14$ $-2.0008 E-14$ 



$\begin{array}{cc}x & \text { THETA } \\ 0.0000 & 1.0106 E-01 \\ .0100 & 1.0106 E-01 \\ .0200 & 1.0104 E-01 \\ .0300 & 1.0102 E-01 \\ .0400 & 1.0098 E-01 \\ .0500 & 1.0093 E-01 \\ .0600 & 1.0088 E-01 \\ .0700 & 1.0081 E-01 \\ .0800 & 1.0073 E-01 \\ .0900 & 1.0065 E-01 \\ .1000 & 1.0055 E-01 \\ .0000 & 9.9053 E-02 \\ .3000 & 9.6699 E-02 \\ .4000 & 9.3658 E-02 \\ .5000 & 9.0159 E-02 \\ .60000 & 8.5360 E-02 \\ .7000 & 8.2434 E-02 \\ .8000 & 7.8505 E-02 \\ .9000 & 7.4666 E-02 \\ 1.0000 & 7.0977 E-02 \\ 2.0000 & 4.4675 E-02 \\ 3.0000 & 3.1533 E-02 \\ 4.0000 & 2.4165 E-02 \\ 5.0000 & 1.9532 E-02 \\ 6.0000 & 1.6369 E-02 \\ 7.0000 & 1.4079 E-02 \\ 8.0000 & 1.2347 E-02 \\ 9.0000 & 1.0992 E-02 \\ 10.0000 & 9.9037 E-03 \\ 20.0000 & 4.9595 E-03 \\ 3000000 & 3.3152 E-03 \\ 40.0000 & 2.4869 E-03 \\ 50.0000 & 1.9898 E-03 \\ 60.0000 & 1.6582 E-03 \\ 70.0000 & 1.4214 E-03 \\ 80.0000 & 1.2437 E-03 \\ 90.0000 & 1.1056 E-03 \\ 100.0000 & 9.9503 E-04 \\ 200.0000 & 4.9753 E-04 \\ 300.0000 & 3.3169 E-04 \\ 400.0000 & 2.4877 E-04 \\ 500.0000 & 1.9901 E-04 \\ 600.0000 & 1.6585 E-04 \\ 700.0000 & 1.4215 E-04 \\ 800.0000 & 1.2438 E-04 \\ 900.0000 & 1.1056 E-04 \\ 1000.0 & 9.9507 E-05 \\ & \end{array}$

THETAP

$-1.0362 E-03$ $-2.0714 E-03$ $-3.1048 E-03$ $-4.1353 E-03$ $-5.1620 E-03$ $-6.1840 E-03$ $-7.2004 E-03$ $-8.2102 E-03$ $-9.2127 E-03$ $-1.0207 E-02$ $-1.9519 E-02$ $-2.7249 E-02$
$-3.3033 E-02$ $-3.6837 E-02$ - $3.6837 E-02$ $-3.9452 \mathrm{E}-02$ $-3.8960 E-02$ $-3.7723 E-02$ $-3.0015 E-02$ $-1.7995 E-02$ $-5.4960 E-03$ $-5.6994 E-03$ $-3.7622 E-03$ $-2.6575 E-03$ $-1.9728 E-03$ $-1.5206 E-03$ $-1.2071 E-03$ $-9.8100 E-04$ $-2.4788 E-04$ $-1.1039 E-04$ $-6.2137 E-05$ $-3.9780 E-05$ $-2.7630 E-05$ $-2.0302 E-05$ $-1.5545 E-05$ $-1.2283 E-05$ $-9.9493 E-06$ $-2.4876 E-06$
$-1.1056 E-06$ $-1.1056 E-06$ $-6.2192 E-07$ $-2.7641 E-07$ $-2.7641 E-07$
$-2.0308 E-07$ $-1.5548 E-07$ $-1.5548 E-07$
$-1.2285 E-07$ $-9.9507 E-08$ $. .5443 E-06$
$1.0175 E-05$ $2.2885 \bar{E}-05$ $4.0662 \equiv-05$ $6.3491 \equiv-05$ $9.1351 E-05$ $1.2422 E-04$ $1.52065-04$ $2.0484 E-04$ $2.5253 E-04$ $9.8789=-04$ $2.1446=-03$ $5.5351=-03$ $5.3624=-03$
$7.2337 E-03$ $7.2337 E-03$ $1.1109=-02$ $1.3005 E-02$ $1.4830 \mathrm{E}-02$ $1.4830 E-02$
$2.7879 E-02$ $3.4424=-02$ $3.8100 E-02$ $4.0413 E-02$ $4.1993 E-02$ $4.3137 E-02$ $4.4003 \equiv-02$ $4.4680 E-02$ $4.5224 E-02$ $4.7691 E-02$ $4.8518 E-02$ $4.8933 E-02$ $4.9182 E-02$ $4.9348 E-02$ $4.9467 E-02$ $4.7557 E-02$ $4.96265-02$ $4.9582 E-0$ $4.9936=-02$ $5.0024 E-02$ $5.0070 E$
$5.0100=-02$ $5.0121 E-02$ $5.0121 E-02$ $5.0152=-02$ $5.0164 E=-02$ $5.0174 E-02$
1. $5246 E-03$

2.0306E-03

$2.5348 \mathrm{E}-03$

$3.0368 E-03$

. $J 35 E-03$

5.J243E-O3

$9.5896 E-03$

$1.3395 \mathrm{E}-02$

$1.6251 E-02$

$1.9156 \mathrm{E}-02$

$1.9563 \mathrm{E}-02$

$1.9239 E-02$

$1.8645 E-02$

$1.7816 E-02$

$1.7816 E-02$
$8.9517 E-03$

$4.9347 E-03$

$2.8447 \mathrm{E}-03$

$1.8789 E-03$

$1.3277 E-03$

$7.5998 E-04$

$6.0335 E-04$

$4.9039 E-04$

$1.2398 \mathrm{E}-0$

$5.5242 E-05$

$3.1117 E-05$

1. $9940 E-05$

$1.3865 \mathrm{E}-05$

$1.0201 E-05$

$7.8220 E-06$

$6.1911 E-06$

$5.0244 E-06$

$1.2935 E-06$

$5.0256 E-07$

$3.6071 E-07$
$2.4877 E-07$

$1.8796 E-07$

$1.8129 E-07$

$1.2749 \mathrm{E}-07$

$9.9507 E-08$
FPP

$5.0889 E-02$

$5.0866 E-02$
$5.0797 E-02$

$5.0681 E-02$

$5.0520 E-02$

$5.0313 E-02$

$5.0061 \mathrm{E}-02$

$4.9765 E-02$
$4.9426 E-02$

$4.9044 E-02$

$4.8620 E-02$

$4.2350 E-02$

$2.3634 E-02$

$2.3634 E-02$

$6.3284 E-03$

$-4.3411 E-03$

$-7.3116 E-03$

$-9.0993 E-03$

$-6.3027 E-03$

$-2.6910 E-03$

$-1.2992 E-03$

$-7.0904 E-04$

$-1.3167 E-04$

$-9.6644 E-05$

-3.6735E-06

$-1.5520 E-05$

$-7.9513 E-07$

$-3.6853 E-09$

$-7.9605 E-10$

$-4.6068 E-10$

$-2.9011 E-10$

$-1.9435 E-10$

$-1.3650 \mathrm{E}-10$

$-9.9507 E-11$
0.

5. $3099 E-06$
$2.1235 E-05$

$4.7760 E-05$

$8.4860 E-05$

$1.3250 E-04$

$1.9064 E-04$
$2.5922 E-04$

$2.5922 E-04$
$3.3817 E-04$

$4.2744 E-04$

$5.2692 E-04$

$2.0600 E-03$

$4.4675 E-03$

..5627E-03

$1.1140 E-02$

$1.8986 \mathrm{E}-02$

$2.2962 \mathrm{E}-02$

$3.0553 E-02$

$5.6774 E-02$

$6.9689 E-02$

$7.6870 E-02$

$8.1363 E-02$

$8.4419 E-02$

$8.6627 E-02$

$8.9596 E-02$

$9.0640 E-02$

$9.5362 E-02$

$9.6940 \mathrm{E}-02$

$9.7729 E-0$

..8203E-02

$9.8518 E-0$

$9.8912 E-0$

$9.9044 E-02$

9.9149E-02

$9.9622 E-02$

$.9858 E-02$

9.9905

$9.9959 E-02$

. $9976=-02$

$9.9989 E-02$

1.0000 E-01
0.

$2.5439 E-04$

$5.0843 E-04$

$7.6178 E-04$

$1.0141 E-03$

$1.5142 E-03$

$1.7614 E-03$

$2.0062 E-03$

$2.4873 E-03$

.

$7.2463 \mathrm{EE}-03$

$7.4138 E-03$

$7.0993 E-03$

$3635 E-03$

$4.1947 E-C 3$

2.1947E-03

$4.9861 E-03$
$-4.9876 E-03$
$-6.9400 E-03$

$-6.6802 E-03$

$-6.2037 E-03$

$-5.6711 E-03$

$-5.176 \mathrm{EE}-03$

$-4.7403 E-03$

$-4.3611 E-03$

$-4.0320 E-03$

$-1.5620 E-0$

$-9.6370 E-04$

$-6.9647 E-04$

- -217 E E 04

$-1.6614 E-04$

1.661 AE-04

$-9.9951 E-05$

-8.334 EE-05

- $14563 E-05$

$-5.0075 E-05$

$2 / A$
5.0889

$5.0882 E-02$

.0 $0858 \mathrm{E}-02$

$5.0820 E-02$

$5.0766 E-02$

$5.0697 E-02$

$5.0613 E-02$

5.0513E-02

S.0399E -02

5.0270E-02

5.0126E-02

$4.7948 E-02$

28E-02

$6277 E-02$

$2.7805 E-02$

$2.4049 E-02$

$1.7816 E-02$

$1.5782 E-03$

$7.1118 E-04$

$2.2128 E-04$

$1.4083 E-04$

. $4998 E-05$

.7039E-05

$6.1990 E-06$ 



$\begin{array}{ccc}\text { X } & \text { THETA } & \text { THETAP } \\ 0.0000 & 5.2461 E-01 & 0 . \\ .0100 & 5.2458 E-01 & -5.9582 E-03 \\ .0200 & 5.2449 E-01 & -1.1910 E-02 \\ .0300 & 5.2434 E-01 & -1.7851 E-02 \\ .0400 & 5.2413 E-01 & -2.3774 E-02 \\ .0500 & 5.2387 E-01 & -2.9673 E-02 \\ .0600 & 5.2354 E-01 & -3.5542 E-02 \\ .0700 & 5.2316 E-01 & -4.1377 E-02 \\ .0800 & 5.2271 E-01 & -4.7171 E-02 \\ .0900 & 5.2221 E-01 & -5.2918 E-02 \\ .1000 & 5.2165 E-01 & -5.8614 E-02 \\ .2000 & 5.1307 E-01 & -1.1167 E-01 \\ .3000 & 4.9965 E-01 & -1.5497 E-01 \\ .4000 & 4.8248 E-01 & -1.8645 E-01 \\ .5000 & 4.6275 E-01 & -2.0514 E-01 \\ .6000 & 4.4159 E-01 & -2.1551 E-01 \\ .7000 & 4.1992 E-01 & -2.1674 E-01 \\ .8000 & 3.9844 E-01 & -2.1209 E-01 \\ .9000 & 3.7763 E-01 & -2.0358 E-01 \\ 1.0000 & 3.5780 E-01 & -1.9277 E-01 \\ 2.0000 & 2.2074 E-01 & -9.1463 E-02 \\ 3.0000 & 1.5464 E-01 & -4.7298 E-02 \\ 4.0000 & 1.1811 E-01 & -2.8128 E-02 \\ 5.0000 & 9.5305 E-02 & -1.8478 E-02 \\ 6.0000 & 7.9793 E-02 & -1.3016 E-02 \\ 7.0000 & 6.8587 E-02 & -9.6450 E-03 \\ 8.0000 & 6.0124 E-02 & -7.4256 E-03 \\ 9.0000 & 5.3510 E-02 & -5.8895 E-03 \\ 10.0000 & 4.8203 E-02 & -4.7834 E-03 \\ 20.0000 & 2.4170 E-02 & -1.2062 E-03 \\ 30.0000 & 1.6122 E-02 & -5.3695 E-04 \\ 40.0000 & 1.2074 E-02 & -3.0220 E-04 \\ 50.0000 & 9.6757 E-03 & -1.9346 E-04 \\ 60.0000 & 8.0535 E-03 & -1.3436 E-04 \\ 70.0000 & 6.9118 E-03 & -9.8724 E-05 \\ 80.0000 & 6.0479 E-03 & -7.5590 E-05 \\ 90.0000 & 5.3760 E-03 & -5.9727 E-05 \\ 100.0000 & 4.8384 E-03 & -4.8381 E-05 \\ 200.0000 & 2.4193 E-03 & -1.2096 E-05 \\ 300.0000 & 1.6129 E-03 & -5.3762 E-05 \\ 400.0000 & 1.2096 E-03 & -3.0241 E-06 \\ 500.0000 & 9.6772 E-04 & -1.9354 E-05 \\ 600.0000 & 8.0543 E-04 & -1.3441 E-06 \\ 700.0000 & 6.9123 E-04 & -9.8747 E-07 \\ 800.0000 & 6.0482 E-04 & -7.5603 E-07 \\ 900.0000 & 5.3762 E-04 & -5.9736 E-07 \\ 1000.00 & 4.8386 E-04 & -4.8386 E-07 \\ & & \end{array}$

0.

$.3592 E-05$ $5.4355 \equiv-05$
$1.2225 E-04$ $1.2225 E-04$
$2.1721 E-04$
$3.3915 E-04$ $3.3915=-04$ $3.3915 E-04$
$4.8796 E-24$ $6.6348 E-04$ $8.6555 \bar{c}-04$ $1.0940 \equiv-03$ $1.3486 \equiv-03$ $5.2696 E-03$ $1.1420 E-02$ $1.9314=-02$ .8418 E 02 $3.8231 \equiv-0$ $4.8330=-02$ $5.8389=-02$ $6.8178 E-02$ $7.7551 E-0$ $1.4342 \Sigma-0$ $1.7582 E-0$ $1.9387=-0$ $2.05195-01$ $2.1291=-01$ . 26015-01 2.2601E-0 $2.28662=01$ $2.44695-01$ $2.4469 E=01$
$2.45715-01$ $2.4792 E-01$ $2.4873=-01$ $2.4931 E-01$ $2.4974 E-01$ $2.5008 \equiv-01$ $2.5035 E-01$ $2.5158=-01$ $2.5201 E-01$ $2.5224 E-01$ $2.5238 E-01$ $2.5249 \vec{E}-01$ $2.5257 E-01$ $2.5264 E-01$ $2.5269 E-01$
$2.5274 E-01$
FP

0.

$.7182 E-03$ $5.4338 \mathrm{E}-03$ $8.1442 E-03$
$1.0847 E-02$ $1.35395-02$ $1.6219 E-02$ $1.8883 E-02$ $2.1529 E-02$ $2.4155 E-02$ $2.675 B E-02$ $5.1077 E-02$ $7.1098 E-02$ $8.5878 \mathrm{E}-02$ $9.5371 E-02$ $1.0018 E-01$ $1.0125 E-01$ $9.9554 E-02$ $9.6000 E-02$ $9.1304 E-02$ $4.4566 E-02$ $2.3314 E-02$ $1.3940 E-02$ 9.1835E-03 $4.8068 E-03$ $4.8068 E-03$ . 2.938 5.0308E-04 . $1.5132 E-04$ $9.6963 E-05$ $6.7420 \varepsilon-05$ $4.9602 E-05$ $3.8036 E-05$ $3.0105 E-05$ $2.4432 E-05$ $6.2900 E-06$ $2.9300 E-06$ $1.7540 E-06$ $1.2096 \mathrm{E}-06$ $9.1395 E-07$ $7.3566 E-07$ $6.1994 E-07$ $5.4061 E-07$ $4.8386 E-07$
FPP

$2.7186 E-01$
$2.7173 E-01$ $2.7173 E-01$
$2.7134 E-01$ $2.7070 E-01$ $2.6979 E-01$ $2.6863 E-01$ $2.6722 E-01$ $2.6555 E-01$ $2.6355 E-01$ $2.6151 E-01$ $2.5913 E-01$ $2.2409 E-01$ 1.7477E-0 1 $1.2079 E-01$ $7.0136 E-02$ $2.7694 E-32$ $-4.7516 E-03$ $-2.7581 E-02$ $-4.2295 E-02$ $-5.0735 E-02$ $-3.2128 E-02$ $-1.3412 E-02$ $-6.4135 E-03$ $-3.4829 E-03$ $-2.0815 E-03$ $-1.3366 E-03$ - $.0680 E-04$ $-6.4242 E-04$
$-4.7124 E-04$ $-6.0085 E-D 5$ - $0868 E-05$ $-7.5479 E-06$ $-3.8668 E-06$ $-2.2385 E-05$ $-2.2385 E-05$
$-1.4099 E-06$ $-9.4455 E-07$ $-6.6352 E-07$ $-4.8373 E-07$ $-6.0478 E-08$ $-1.7920 E-08$ $-7.5602 E-09$ $-3.8708 E-09$ $-2.2401 E-09$ $-1.4107 E-09$ $-9.4503 E-10$ $-6.6373 E-10$ $-4.8386 E-10$
5 $1.3340 E-04$ $3.0001 E-04$ $5.3302 E-04$
$8.3217 E-04$ $8.3217 E-04$
$1.1971 E-03$ $1.6275 \mathrm{E}-03$ $2.1229 \mathrm{E}-03$ 2. 6827 E-0 3 $3.3064 E-03$ 1.2881 E-02 $2.7784 E-02$ 4. $6706 \mathrm{E}-02$ $6.8243 E-02$ $9.1109 E-02$ $1.1427 E-01$ $1.5867 E-01$ $1.7913 \mathrm{E}-01$ $3.1362 E-01$ $3.7377 E-01$ $4.0554 E-01$ $4.2482 E-01$ $4.3768 E-01$ $4.4684 E-01$ $4.5368 E-01$ $4.5398=01$ $4.8201 E-01$ $4.8819 E-01$ 4. $9125 E-0$
4. $9308 E-01$ $4.9308 E-01$
$4.9430 E-01$ $4.9430 E-01$ $4.9517 E-01$
$4.9582 E-01$ $4.9582 E-01$ $4.9673 E-01$ $4.9855 E-01$ $4.9916 E-01$ $4.9946 E-01$

4.9976E-01

$4.9984 E-01$

$4.9991 E-0$

4. $9996 E-01$
$5.0000 E-01$
Z/A

(1.

$1.3590 E-03$ $4.0692 E-03$ $5.4165 E-03$ $6.7561 \mathrm{E}-03$ $8.0860 E-03$ $9.4045 E-03$ $1.0710 E-02$ $3.3033 E-02$ $3.7594 E-02$ $3.8534 E-02$ $3.6463 E-02$

J.220JE-02

$2.024 E E-02$

1. $3753 \mathrm{E}-02$ $-2.7143 E-02$ $-3.5292 E-02$ उ. 2.900 SE -02 $-2.413 \subseteq E-02$ $-2.413 \mathrm{EE}-02$ $-2.0478 E-02$ $-1.1430 \mathrm{E}-02$ $-4.8614 E-03$ $-4.0780 E-03$ $-3.511 \mathrm{CE}-03$ $-3.0837 E-03$ $-2.7485 E-03$ $-2.4791 E-03$ -1.251 EE- 03 $-8.3711 E-04$ $-5.0355 E-04$ $-4.1990 E-04$ $-3.6008 E-0$ $3.1517 E-04$
$-2.8023 E-04$ $-2.5226 E-04$
Z/A H

$2.7186 E-01$
$2.7182 E-01$
$2.7169 E-01$ $2.7182 E-01$
$2.7169 E-01$
$2.7147 E-01$ 2.7117E-01 $2.7078 E-01$ 2.7031E-01 $2.6975 E-01$ $2.6911 E-01$ $2.6839 E-01$ $2.6758 E-01$ $2.5538 E-01$ $2.3699 E-01$ . $1469 E-01$ $1.9074 E-0$

$1.6697 E-01$

$1.2444 E-01$

$1.0667 E-01$

$9.1304 E-02$ $2.2283 E-02$ 7.7715E-0 $3.4849 E-03$ $1.8367 E-03$ $6.8669 E-04$ $4.6293 E-04$ $3.2654 E-04$ 3. $0154 E-05$ $8.9554 E-06$ $3.7831 E-06$ $1.9393 E-06$ $1.1237 E-06$ 7.0860E-07 $4.7545 E-07$ $3.3450 E-07$ $2.4432 E-07$ 作 作 $3849 E-0$

$.4193 E-0$

$1.5233 E-09$

$1.0509 \mathrm{E}-09$

7.7493E-10 $4.8386 E-10$

2/A DWDR

$-8.6756 E-03$ $-1.7337 E-02$
$-2.5972 E-02$ $-2.5972 E-02$
$-3.4565 E-02$ $-5.1575 E-02$ $-5.9966 E-02$ $-6.8263 E-02$ $-7.6454 E-02$ $-8.4527 E-02$ $-2.0740 E-01$ $-2.3477 E-01$ $-2.4121 E-01$ $-2.3213 E-01$ $-2.1341 E-01$ $-1.9003 E-01$ $-1.4204 E-01$ $-2.47465-03$ $-1.0639 E-03$ 


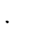




\begin{tabular}{|c|c|c|}
\hline .0000 & THETA & THETAP \\
\hline 0.0000 & $1.0959 E+00$ & 0 \\
\hline .0100 & $1.0958 E+00$ & $-1.4185 E-02$ \\
\hline $\begin{array}{l}.0200 \\
.0300\end{array}$ & $.0956 E+00$ & $-2.8354 E-02$ \\
\hline .0300 & $.0953 E+00$ & $-4 \cdot 2492 E-02$ \\
\hline .0400 & $1.0948 E+00$ & $-5.6583 E-02$ \\
\hline .0500 & $.0941 E+00$ & $-7.0511 E-02$ \\
\hline .0600 & $1.0934 E+0 C$ & $-8.4563 E-02$ \\
\hline .0700 & $1.0924 E+00$ & $-9.8422 E-02$ \\
\hline .0800 & $1.0914 E+00$ & $-1.1217 E-01$ \\
\hline .0900 & $1.0902 E+00$ & $-1.2580 E-01$ \\
\hline .1000 & $1.0889 E+00$ & $-1.3930 E-01$ \\
\hline .2000 & $1.068 \mathrm{JE}+00$ & $-2.6403 E-01$ \\
\hline .3000 & $1.0369 E+00$ & $-3 \cdot 6351 E-01$ \\
\hline .4000 & $9.9683 E-01$ & $-4.3298 E-01$ \\
\hline .5000 & $9.5129 E-01$ & $-4.7322 E-01$ \\
\hline .6000 & $9.0301 E-01$ & $-4.8873 E-01$ \\
\hline .7000 & $8.5417 E-01$ & $-4.8549 E-01$ \\
\hline .8000 & $8.0634 E-01$ & $-4.6940 E-01$ \\
\hline .9000 & $7.6056 E-01$ & $-4.4540 E-01$ \\
\hline 1.0000 & $7.1740 E-01$ & $-4.1726 E-01$ \\
\hline 2.0000 & $4.3085 E-01$ & $-1.8507 E-01$ \\
\hline 3.0000 & $2.9888 E-01$ & $-9.3240 E-02$ \\
\hline 4.0000 & $2.2732 E-01$ & $-5.4803 E-02$ \\
\hline 5.0000 & $1.8302 \mathrm{E}-01$ & $-3.5780 E-02$ \\
\hline 6.0000 & $1.5303 E-01$ & $-2.5113 E-02$ \\
\hline 7.0000 & $1.3144 E-01$ & $-1.8567 E-02$ \\
\hline 8.0000 & $1.1516 E-01$ & $-1.4273 E-02$ \\
\hline 9.0000 & $1.0245 E-01$ & $-1.1308 \mathrm{E}-02$ \\
\hline 10.0000 & $9.2267 E-02$ & $-9.1774 E-03$ \\
\hline 20.0000 & $4.6225 E-02$ & $-2.3083 E-03$ \\
\hline 30.0000 & $3.0827 E-02$ & $-1.0270 E-03$ \\
\hline 40.0000 & $2.3123 E-02$ & $-5.7790 E-04$ \\
\hline 50.0000 & $1.8500 E-02$ & $-3.6992 E-04$ \\
\hline 60.0000 & $1.5417 E-02$ & $-2.5691 E-04$ \\
\hline 70.0000 & $1.3215 E-02$ & $-1.8876 E-04$ \\
\hline & $1.1563 E-02$ & $-1.4453 E-04$ \\
\hline 90.0000 & $1.0278 E-02$ & $-1.1420 E-04$ \\
\hline 100.0000 & $9.2506 E-03$ & $-9.2501 E-05$ \\
\hline & & $-2.3127 E-05$ \\
\hline 10.0000 & $3.0836 E-1$ & $-1.0279 E-05$ \\
\hline 400.0000 & $2.3127 E-03$ & $-5.7817 E-0 E$ \\
\hline & $1.8502 E-$ & $-3.7003 E-0$ \\
\hline & $1.5418 E-03$ & $-2.5697 E-0$ \\
\hline 700.0000 & $1.3215 E-03$ & $-1.8879 E-06$ \\
\hline & $1.1563 E-03$ & $-1.4454 E-06$ \\
\hline & $1.0279 E-03$ & $-1.1421 E-$ \\
\hline 1000.0 & $9.2508 E-04$ & $-9.2508 E-07$ \\
\hline
\end{tabular}

$F$

$2.9456 E-05 \quad 5.8907 E-03$ $1.1779 E-04 \quad 1.1775 E-02$ $2.6492 E-04 \quad 1.7648 E-02$ 4.7069E-04 2.3503E-02 $7.3490=-04 \quad 2.9333 E-02$ $1.0375=-03 \quad 3.5134 E-02$ $1.8751=-03 \quad 4.0902 E=02$ 2.3698E-03 $5.2302 E=02$ $2.9210=-03 \quad 5.7927 E-02$ $1.13965-02 \quad 1.1023 E=01$ $2.46375=02 \quad 1.5270 E=01$ $4.1539=-02 \quad 1.8333 E-01$ $6.0908 E-02 \quad 2.0219 E-01$ $8.1636 E-02 \quad 2.1085 E-01$ $1.0281 E-01 \quad 2.1153 E-01$ $1.2375 E-01 \quad 2.0652 E-01$ $1.4399 E-01 \quad 1.9781 E-01$ $1.6324 E-01 \quad 1.8696 E-01$ $2.9545 E-01 \quad 8.7828 E-02$ $3.5883 E-01 \quad 4.5296 E-02$ $3.9379 E-01 \quad 2.5913 E-02$ $4.1561 E-01 \quad 1.7673 E-02$ $4.3045 E-01 \quad 1.2447 E-02$ $4.4116=-01 \quad 9.2223 E-03$ $4.4925=-01 \quad 7.0999 E-03$ $4.5558 E-01 \quad 5.6310 E-03$ $4.6065 E-01 \quad 4.5735 E-03$ $4.8363 E-01 \quad 1.1535 E-03$ $4.9133 E-01 \quad 5.1375 E-04$ $4.9519 E-01 \quad 2.8935 E-04$ $4.9750 E-01 \quad 1.8539 E-04$ $5.905=01 \quad 1.2891=-04$ $5.0015=-01 \quad 9.4837=05$ $5.0163 E-01 \quad 5.7558 E-05$ $5.0215=-01$. $5.0451=-01 \quad 1.2026 E-05$ $5.0532 E-01 \quad 5.6018 E-06$ $5.0576=-01 \quad 3.3534 E-06$ $5.0603 E-01 \quad 2.3127 E-06$ $5.0623 E-01 \quad 1.7474 E-06$ $5.0639=-01 \quad 1.4065 E-06$ $5.0652 E-01 \quad 1.1853 E-06$ $5.0663 E-01 \quad 1.0336 E-06$ $5.0673 E-01$
FPP

$5.8917 E-01$
$5.8887 E-01$ $5.8797 E-01$ $5.8646 E-01$ $5.8437 E-01$ $5.8168 E-01$ $5.7842 E-01$ $5.7458 E-01$ $5.7018 E-01$ $5.5974 \mathrm{E}-01$ $4.7918 \mathrm{E}-01$ $2.4580 E-01$ $1.3421 E-01$ $4.2776 E-02$ $-2.5272 E-02$ $-7.1564 E-02$ $-1.0004 E-01$ $-1.1515 E-01$ $-6.5245 E-02$ $-2.6462 E-02$ $-1.2499 E-02$ $-6.7449 E-03$ $-4.0163 E-03$ $-2.5731 E-03$ $-1.7430 E-03$ $-1.2335 E-03$ $-9.0413 E-04$ $-1.1498 E-04$ $-3.4176 E-0$ $-1.4434 E-0$ $-7.3940 E-05$ $-4.2801 E-05$ $-2.6958 E-06$ $-1.8062 E-05$ $-1.2686 E-05$ $-1.1563 E-07$ $-3.4261 E-08$ $-1.4454 E-0 B$
$-7.4006 E-09$ $-7.4006 E-09$
$-4.2827 E-09$ $-4.2827 E-09$
$-2.6970 E-09$ $-2.6970 E-09$
$-1.8068 E-09$ $-1.2690 E-09$ $-9.2508 \mathrm{E}-10$
$8.7069 E-05$ $3.4817 E-0$ $7.8297 E-0$ $1.3909 \mathrm{E}-03$ $2.1713 \mathrm{E}-03$ $3.1231 E-03$ $5.53595-03$ 6. $8.61777 E-03$ $3.3433 E-02$ $7.1640 E-02$ $1.1942 E-01$ $1.7281 E-01$ $1.7281 E-01$
$2.2833 E-0$ $2.2833 E-01$
$2.8332 E-01$ $2.8332 E-01$
$3.3601 E-01$ $3.8531 E-01$ $4.3074 E-01$
$7.0416 E-01$ $8.1133 E-0$ $8.6377 \mathrm{E}-0$ $8.9409 E-01$ $9.1364 E-0$ $9.2722 E-0$ $9.3718 E-0$ $9.4478 E-0$ $9.5077 E-0$ $9.7660 E-01$ $9.8478 \mathrm{E}-0$ $9.8878 E-01$ 9.9116E-01 9. $9273 E-01$ $9.9369=01$ $9.9469 E-01$ 9.953E-01 9.9 $9.9893 E-01$ $9.9931 E-01$ $9.9954 E-01$ $9.9970 E-01$ $9.9970 E-01$ $9.9980 E-01$ $9.9995 E-0$ $1.0000 E+00$
Z/A U $2.9451 E-03$ 5.885TE-03 $1.173 \mathrm{EE}-02$ $1.463 .5 E-02$ $1.7513 E-02$ 2.036 EE 02 $2.5971 \mathrm{E}-02$ 2. $5.324 \mathrm{CE}-02$ $7.0580 E-02$ $7.9477 \mathrm{E}-0$ $8.0373 E-02$ $8.0373 E-02$
$7.4788 E-02$ $6.4656 \mathrm{E}-0$ $5.1830 E-02$ $3.7819 E-02$ $2.3714 E-02$ $-5.989 \mathrm{EE}-0$ $-7.4314 \mathrm{E}-02$ $-7.1535 E-02$ $-6.5449 E-02$ IE $-5.3801 E-02$ $-4.4989 E-02$ $-4.1492 E-02$ $-2.3028 E-02$ $-1.5864 E-02$ $-1.2090 \mathrm{OE}-02$ $-9.7646 E-03$ $-8.1885 E-03$ $-7.0502 E-03$ $-5.5161 E-03$ $-4.9748 E-03$ $-2.5105 E-0$ $-1.6788$ $-1.2610 E-03$ $-1.0098 E-03$ $-6.3196 E-0$ $-5.6189 E-04$
Z/A

5.8917E-01 5.8907E-01 (5.887 E-01 . (5.8657E-01 S (5) $5.7927 E-01$ 5.5115E-01 .0901E-01 $4.5831 E-01$ $3.5141 \mathrm{E}-01$ $3.0219 E-01$ .5815E-01 $2.1979 E-01$ .8696E-01 1.5 $1099 E-02$ $6.7283 E-03$ 3.5347E-03 $1.3175 E-03$ 8.8748E-04 $6.2567 E-04$ $4.5735 E-04$
$5.7677 E-05$ $5.7677 E-05$
$1.7125 E-05$ $7.2337 E-06$ $3.7079 E-06$ $2.1484 E-06$ $1.3548 E-06$ $6.3954 E-07$ $4.6711 E-07$ $.0128 \mathrm{E}-08$ $.8673 E-08$ $4.6254 E-09$ $2.9123 E-09$ 2.0093E-09 $1.4816 E-09$ $1.4816 E-09$ $9.2508 E-10$
22/A DHDR $-4.0113 E-02$ -7. $-1.3864 E-01$ $-1.5779 E-01$ -1 -7667E-01 $-1.9527 \mathrm{E}-01$ $-3.5987 E-01$ $-4.7359 E-01$ $-5.3129 E-01$ $-5.4033 E-01$ $-5.1440 E-01$ $-4.6780 E-01$ $-4.1214 E-01$ $-3.5537 E-01$ $-3.0211 E-01$ $-5.4579 E-02$ $-1.3854 E-02$ $4.8068 E-03$ $-2.0559 E-03$ $-1.0151 E-03$ $-3.2881 E-04$ $-2.0658 E-04$ $-1.3615 E-04$ $-5.4169 E-07$ $-1.0714 E-07$ $-5.7866 E-08$ -3. $2.1202 E-08$ 1. $3920 E-08$ $-8.7878 \mathrm{E}-10$ $-1.7645 E-10$ $-5.7094 E-11$ $-2.4052 E-11$ $-1.1992 E-11$ $-6.7233 E-12$ $-4.1104 E-12$ $-2.6860 E-12$ 


\begin{tabular}{|c|c|c|c|}
\hline$x$ & & $\begin{array}{l}\text { THETAP } \\
0 .\end{array}$ & $0 .{ }^{F}$ \\
\hline $\begin{array}{r}0.0000 \\
.0100\end{array}$ & $\begin{array}{l}6.0983 E+00 \\
6.0974 E+00\end{array}$ & $\begin{array}{c}0 . \\
-1.8666 E-01\end{array}$ & $\begin{array}{l}0.0616 \equiv-04 \\
2.0616\end{array}$ \\
\hline & $6.0946 E+00$ & $-3.7293 E-01$ & $8.2429 E-04$ \\
\hline .0300 & $6.0700 E+D 0$ & $-5.5843 E-01$ & $1.8534=-03$ \\
\hline .0400 & $6.0835 E+00$ & $-7.4278 E-01$ & $3.2917=-03$ \\
\hline .0500 & $6.0751 E+00$ & $-9.2559 E-01$ & $5.1369 \equiv-03$ \\
\hline .0600 & $6.0649 E+00$ & $-1.1065 E+00$ & $7.3860 E-03$ \\
\hline $\begin{array}{l}.0700 \\
.0800\end{array}$ & $\begin{array}{l}6.0530 E+00 \\
6.0373 E+00\end{array}$ & $\begin{array}{l}-1.2852 E+00 \\
-1.4612 E+00\end{array}$ & $\begin{array}{l}1.0035 E-02 \\
1.3080 E-02\end{array}$ \\
\hline .0900 & $6.0238 E+00$ & $-1.6344 E+00$ & $1.6516 \bar{E}-02$ \\
\hline .1000 & $6.0066 E+00$ & $-1.8042 E+00$ & $2.0338 \equiv-02$ \\
\hline .2000 & $5.7491 E+00$ & $-3.2661 E+00$ & $7.8165 E-02$ \\
\hline .3000 & $5.3715 E+00$ & $-4.1911 E+00$ & $1.6514 E-01$ \\
\hline .4000 & $4.9291 E+00$ & $-4.5745 E+00$ & $2.7062 E-01$ \\
\hline .5000 & $4.4706 E+00$ & $-4.5385 E+00$ & $3.8450 \equiv-01$ \\
\hline .6000 & $4.0300 E+00$ & $-4.2418 E+0 D$ & $4.7888 \equiv-01$ \\
\hline .7000 & $3.6264 E+00$ & $-3.8195 E+00$ & $6.0856 E-01$ \\
\hline .8000 & $3.2672 E+00$ & $-3.3634 E+00$ & $7.1061 \equiv-01$ \\
\hline .9000 & $2.9531 E+00$ & $-2.9255 E+00$ & $8.0379 E-01$ \\
\hline 1.0000 & $2.6807 E+00$ & $-2.5304 \varepsilon+00$ & $8.8792 E-01$ \\
\hline 2.0000 & $1.3065 E+00$ & $-7.0223 E-01$ & $1.3749 E+00$ \\
\hline 3.0000 & $8.4739 E-01$ & $-2.9854 E-01$ & $1.5691 E+00$ \\
\hline 4.0000 & $6.2683 E-01$ & $-1.6288 \bar{c}-01$ & $1.6694 E+00$ \\
\hline 5.0000 & $4.9773 E-01$ & $-1.0234 E-01$ & $1.7301 \equiv+00$ \\
\hline 6.0000 & $4.1294 E-01$ & $-7.0257 \mathrm{E}-02$ & $1.7707 \equiv+00$ \\
\hline 7.0000 & $3.5295 E-01$ & $-5.1228 E-02$ & $1.7998 E+00$ \\
\hline 8.0000 & $3.0824 E-01$ & $-3.9017 E-02$ & $1.8216 E+00$ \\
\hline 9.0000 & $2.7363 E-01$ & $-3.0714 E-02$ & $1.8386 E+00$ \\
\hline 10.0000 & $2.4602 E-01$ & $-2.4810 E-02$ & $1.8522 \equiv+00$ \\
\hline 20.0000 & $1.2261 \mathrm{E}-01$ & $-6.1444 E-03$ & $1.9134 E+00$ \\
\hline 30.0000 & $8.1687 E-02$ & $-2.7257 E-03$ & $1.9338 \equiv+00$ \\
\hline 40.0000 & $6.1251 E-02$ & $-1.5322 E-03$ & $1.9440 \equiv+00$ \\
\hline 50.0000 & $4.8996 E-02$ & $-9.8029 E-04$ & $1.9502 E+00$ \\
\hline 60.0000 & $4.0827 E-02$ & $-6.8064 E-04$ & $1.9543 E+00$ \\
\hline 70.0000 & $3.4994 E-02$ & $-5.0001 E-04$ & $1.9572 E+00$ \\
\hline 80.0000 & $3.0619 E-02$ & $-3.8279 E-04$ & $1.9594 E+00$ \\
\hline 90.0000 & $2.7216 E-02$ & $-3.0244 E-04$ & $1.9611 E+00$ \\
\hline 100.0000 & $2.4494 E-02$ & $-2.4497 E-04$ & $1.9625 \equiv+00$ \\
\hline 200.0000 & $1.2247 \mathrm{E}-02$ & $-6.1235 E-05$ & $1.9687 E+00$ \\
\hline 300.0000 & $8.1644 E-03$ & $-2.7215 E-05$ & $1.9709 E+00$ \\
\hline 400.0000 & $6.1233 E-03$ & $-1.5308 E-05$ & $1.9720 E+00$ \\
\hline 500.0000 & $4.8986 E-03$ & $-9.7973 E-06$ & $1.9728 E+00$ \\
\hline 600.0000 & $4.0822 E-03$ & $-6.80 \leq 7 E-06$ & $1.9733 E+00$ \\
\hline 700.0000 & $3.4990 E-03$ & -4.998ठБE -06 & $1.9737 E+00$ \\
\hline 800.0000 & $3.0616 E-03$ & $-3.8271 E-06$ & $1.9741 \equiv+00$ \\
\hline 900.0000 & $2.7215 E-03$ & $-3.0238 E-06$ & $1.9743 E+00$ \\
\hline 1000.0 & $2.4493 E-03$ & $-2.4493 E-06$ & $1.9746 E+00$ \\
\hline
\end{tabular}

0.

. $1226 E-02$ $8.2384 E-02$ $1.2341 E-01$ $1.5422 E-01$
$2.0477 E-01$ $2.4498 E-01$ 2.8479E- 01 $3.2414 E-01$ $3.6297 E-01$ $4.0122 E-01$ $7.4090 E-01$ $.8037 E-01$ 1. $1121 E+00$ $1.1522 c+00$ $1.1269 \bar{E}+00$ $1.0619 E+00$ $9.7718 E-01$ 8.8620E-01 7. $9699 E-01$ $2.8273 E-01$ $1.3258 E-01$ $1.5666 E-02$
$4.8690 E-02$ $4.8690=02$ $3.3897=02$ 1. $1.5103 E-02$ $1.22375-02$ $1.0625 E-03$ $1.3619 E-03$ $7.6662 E-04$ $4.9108 E-04$ $3.4140 E-04$ $2.5115 E-04$ $1.9258 E-04$ $1.5242 E-04$ $1.2369 E-04$ $3.1841 E-05$ $1.4832 \mathrm{E}-05$ 8. $3788 E-06$ $6.1233 E-06$ $4.6265 E-06$ $3.7240 E-06$ $3.1382 E-06$ $2.7366 E-06$
$2.4493 E-06$
FPP

$4.1237 E+00$ $4.1203 E+00$
$4.1101 E+00$ $4.0931 E+00$ $4.0694 E+00$ $4.0390 E+00$ $4.0022 E+00$ $3.9591 E+00$ $3.9099 E+00$ $3.8547 E+00$ $3.7938 E+00$ $2.9339 E+00$ $1.8446 E+00$ $8.1936 E-0$ 2.8 107E-02 $-4.9038 E-01$ $-7.7630 E-01$ $-8.9586 E-01$ $-9.1065 E-01$ $-8.6672 E-01$ $-2.5262 E-01$ $-8.5142 E-02$ $-3.7211 E-02$ $-1.9306 E-02$ $-7.1006 E-03$ $-7.1006 E-03$
$-4.7654 E-03$ $-4.7654 E-03$ $-2.4443 E-03$ $-3.0607 E-04$ $-3.0507 E-04$
$-9.0706 E-05$ $-3.8269 E-05$ $-1.9594 E-05$ $-1.1339 E-05$ $-7.1408 E-06$ $-4.7838 E-05$ $-3.3598 E-06$ $-2.4493 E-05$ $-3.0616 E-07$ $-9.0715 E-08$ $-3.3270 E-08$ $-1.9594 E-08$ $-1.1339 E-08$ $-7.1408 E-09$ $-4.7838 E-09$ $-3.3598 E-09$ $-2.4493 E-09$
0.

$1.5620 E-03$
$6.2438 E-03$ $6.2438 E-03$ $1.4033 E-02$
$2.4910 E-02$ $3.8847 \mathrm{E}-02$ $3.8847 E-02$
$5.5806 E-02$ 7.5745E-02 $9.8613 E-02$ $1.2435 E-01$ $1.5290 \mathrm{E}-01$ $5.7453 E-01$ $1.1726 E+00$ $1.8410 E+00$ $2.4939 E+00$ $3.0798 \mathrm{E}+00$ $3.5773 \mathrm{E}+00$ $3.9851 E+00$ $4.3120 E+00$ $4.5705 E+00$ $5.4116 E+00$ $5.4471 E+00$ $5.4009 E+00$ 3. $3518 E+00$ $5.3102 E+00$ 5. $2483 E+0 O$ $5.2252 E+0$ $5.2059 E+00$ $5.1094 E+00$ $5.0738 E+00$ $5.0554 E+00$ $5.0442 E+00$ $5.0366 E+00$ $5.0270 E+00$ $5.0238 E+00$ $5.0212 E+00$ $5.0095 E+00$ $5.0055 E+00$ $5.0036 E+00$ $5.0024 E+0$ $5.0016 E+00$ $5.0010 E+00$ $5.0006 E+00$

$5.0003 E+00$
ZlA

2.

$2.0610 E-02$ $4.1165 E-02$ $8.1930 E-02$ $1.0203 E-01$ $1.2188 E-01$ $1.4143 E-01$ $1.6064 E-01$ $1.7946 E-01$ $1.9784 E-01$
$3.5007 E-0$

$4.2991 \mathrm{E}-0$

$4.3555 E-01$

$3.8321 \mathrm{E}-01$

$2.9543 E-01$

$1.9253 \mathrm{E}-01$ $8.8916 E-02$ $-6.9028 E-03$ $-4.0474 E-O 1$ $-4.0474 E-C 1$ $-3.4167 E-01$ $-2.6122 E-01$ $-2.3217 E-01$ $-2.0859 E-01$ $-1.8918 \mathrm{E}-0$ -1.729 EE - 0 $-9.2607 E-02$ $-6.3099 E-02$ $-4.7834 E-02$ $-3.8512 E-02$ $-3.2230 E-02$ $-2.7709 E-0$ $-2.4300 E-02$ $-2.1638 E-02$ $-1.9501 E-02$ $-9.811 \mathrm{gE}-03$ $-6.5548 E-03$ $-4.9212 E-03$ $-3.2842 E-03$ $-2.8159 E-03$ $-2.4644 E-03$ $-2.1910 E-03$ $-1.9722 E-03$
Z/A H

$4.1237 E+00$ $4.1226 E+00$ $4.1135 E+00$ $4.1056 E+00$

$4.0954 E+00$

$4.0830 E+00$

$4.0685 E+00$

$4.0518 E+00$

$4.0330 E+00$

$4.0122 E+00$

$3.2679 E+00$ $2.7803 E+0 C$

$2.3044 E+00$

$1.8782 E+0$

$1.5170 E+00$

$1.2215 E+00$

$9.8467 E-01$

$7.9699 E-01$

$1.4137 E-01$

$4.4192 E-02$

$1.8917 E-02$

$9.7381 E-03$

$3.5623 E-03$

2.3882E-0

$1.6781 E-03$

1.5312E-04

$1.9165 E-05$

$9.8216 E-06$

$5.6901 E-06$

$3.5879 E-06$ $2.4072 E-06$ $1.6935 \mathrm{E}-06$ $1.2369 E-06$ $1.5921 E-07$ $4.9440 E-0 B$ $2.2197 E-08$ $1.2247 E-08$ $7.7108 \mathrm{E}-09$ S.319ge 09 3.9227E-09

$2.4493 E-09$
$22 / A$ DWDR

$-2.2786 E-01$ $-4.5513 E-01$ $-9.0555 E-01$ $-1.1275 E+00$ $-1.3466 E+00$ $-1.5623 E+00$ $1.7740 E+00$ - $9813 E+00$ $-3.8528 E+00$ $-4.9022 E+00$ $-4.5526 E+00$ $-3.9476 E+00$ $-3.2761 E+00$ $-2.6467 E+00$ $-2.1059 E+00$ $-4.3111 E-02$ $-4.3111 E-02$ $-1.4032 E-02$ $-2.8149 E-03$ -1.5233E-0 $-8.9420 E-04$ $-5.5874 E-04$ $-3.6680 E-04$ $-2.2960 E-05$ $-2.8382 E-07$ $-1.5327 E-07$ $-8.9887 E-08$ $-5.6148 E-08$ $-3.6862 E-08$ $-4.6718 E-10$ $-1.5117 E-10$ $-6.3682 E-11$ $-1.0883 E-11$ $-4.8986 E-12$ 


\begin{tabular}{|c|c|c|c|c|c|}
\hline$x$ & THETA & THETAP & $\boldsymbol{F}$ & & FPP \\
\hline 0.0000 & $1.1213 E+01$ & 0 & 0. & 0 & $8.5489 E+00$ \\
\hline .0100 & $1.1210 E+01$ & $-5.9111 E-01$ & $4 \cdot 2736 E-04$ & $8.5456 E-02$ & $8 \cdot 5388 E+00$ \\
\hline .0200 & $1.1201 E+01$ & $-1.1804 E+00$ & $1.7084 E-03$ & $1.7071 \mathrm{E}-01$ & $8.5084 E+0 C$ \\
\hline .0300 & $1.1186 E+01$ & $-1.7659 E+00$ & $3.8402 E-03$ & $2.5556 E-01$ & $8.4580 E+00$ \\
\hline .0400 & $1.1166 E+01$ & $-2 \cdot 3460 E+00$ & $6.8176:-0.3$ & $3.3980 E-01$ & $8.3878 E+00$ \\
\hline .0500 & $1.1140 E+01$ & $-2.9187 E+00$ & $1.0634 \Xi-02$ & $4.2325 E-01$ & $8.2983 E+00$ \\
\hline .0600 & $1.1107 E+01$ & $-3.4825 E+00$ & $1.5279 E-02$ & $5.0571 E-01$ & $8.1900 E+00$ \\
\hline .0700 & $1.1070 E+01$ & $-4.0356 E+00$ & $2.0744 \Xi-02$ & $5.8699 E-01$ & $8.0635 E+00$ \\
\hline .0800 & $1.1027 E+01$ & $-4.5764 E+00$ & $2.7014=-02$ & $6.6692 E-01$ & $7.9197 E+00$ \\
\hline .0900 & $1.0978 E+01$ & $-5.1035 E+00$ & $3.4077 E-02$ & $7.4533 E-01$ & $7.7592 E+00$ \\
\hline .1000 & $1.0925 E+01$ & $-5.6155 E+0 O$ & $4.1915 \equiv-02$ & $8.2205 E-01$ & $7.5831 E+00$ \\
\hline .2000 & $1.0143 E+01$ & $-9.6774 E+00$ & $1.5846 \equiv-01$ & $1.4687 E+00$ & $5.2000 E+00$ \\
\hline .3000 & $9.0635 E+00$ & $-1 \cdot 1551 E+01$ & $3.2673 E-01$ & $1.8512 E+C 0$ & $2.4823 E+00$ \\
\hline .4000 & $7.8948 E+00$ & $-1.1575 E+01$ & $5.2028 E-0.1$ & $1.9831 E+00$ & $2.8266 E-01$ \\
\hline .5000 & $6.7849 E+00$ & $-1.0502 E+01$ & $7.1736 E-01$ & $1.9353 E+00$ & $-1.1041 E+00$ \\
\hline .6000 & $5.8085 E+00$ & $-8 \cdot 9974 E+00$ & $9.039 .9 E-01$ & $1.7861 E+00$ & $-1.7806 E+00$ \\
\hline .7000 & $4.9859 E+00$ & $-7.4727 E+00$ & $1.0732 E+00$ & $1.5950 E+00$ & $-1.9814 E+00$ \\
\hline .8000 & $4.3082 E+00$ & $-6 \cdot 1170 E+00$ & $1.2228 E+00$ & $1.3985 E+00$ & $-1.9179 E+00$ \\
\hline .9000 & $3.7549 E+00$ & $-4 \cdot 9867 E+00$ & $1.3534 \equiv+00$ & $1.2154 E+00$ & $-1.7335 E+00$ \\
\hline 1.0000 & $3.3036 E+00$ & $-4.0743 E+00$ & $1.4666 \bar{E}+00$ & $1.0531 E+00$ & $-1 \cdot 5106 E+00$ \\
\hline 2.0000 & $1.3879 E+00$ & $-8.3979 E-01$ & $2.0509 \equiv+00$ & $3.0990 E-01$ & $-3.0492 E-01$ \\
\hline 3.0000 & $8.6469 E-01$ & $-3.2448 E-01$ & $2.2577 E+00$ & $1.3752 E-01$ & $-9.2760 E-02$ \\
\hline 4.0000 & $6.2983 E-01$ & $-1.7006 E-01$ & $2.3605 E+00$ & $7.6781 E-02$ & $-3.8885 E-02$ \\
\hline 5.0000 & $4.9642 E-01$ & $-1.0471 E-01$ & $2.4218 E+00$ & $4.8880 E-02$ & $-1.9761 E-02$ \\
\hline 6.0000 & $4.1018 E-01$ & $-7.1065 E-02$ & $2.4624 E+00$ & $3.38 \cdot 25 E-02$ & $-1.1372 E-02$ \\
\hline 7.0000 & $3.4972 E-01$ & $-5.1450 E-02$ & $2.4914 \sum+00$ & $2.4792 E-02$ & $-7.1321 E-03$ \\
\hline 8.0000 & $3.0492 E-01$ & $-3.9003 E-02$ & $2.5131 E+00$ & $1.8950 E-02$ & $-4.7639 E-03$ \\
\hline 9.0000 & $2.7038 E-01$ & $-3.0602 E-02$ & $2.5299 E+00$ & $1.4955 E-02$ & $-3.3385 E-03$ \\
\hline 10.0000 & $2.4291 E-01$ & $-2.4662 E-02$ & $2.5433 E+00$ & $1.2103 E-02$ & $-2.4298 E-03$ \\
\hline 20.0000 & $1.2074 E-01$ & $-6.0614 E-03$ & $2.6038 \equiv+00$ & $3.0172 E-03$ & $-3.0194 E-04$ \\
\hline 30.0000 & $8.0407 E-02$ & $-2.6850 E-03$ & $2.6239 \equiv+00$ & $1.3408=-03$ & $-8.9353 E-0 j$ \\
\hline 40.0000 & $6.0281 E-02$ & $-1.5086 E-03$ & $2.6339 E+00$ & $7.5456 E-04$ & $-3.7679 E-05$ \\
\hline 50.0000 & $4.8216 E-02$ & $-9.6495 E-04$ & $2.5400 \equiv+00$ & $4.8330 E-04$ & $-1.9287 E-0 J$ \\
\hline & $4.0176 E-02$ & $-6.6990 E-04$ & $2.6440 E+00$ & $3.3597 E-04$ & $-1.1160 E-05$ \\
\hline 70.0000 & $3.4434 E-02$ & $-4 \cdot 3209 E-04$ & $2.6469 E+00$ & $2.4715 E-04$ & $-7.0276 E-05$ \\
\hline 80.0000 & $3.0129 E-02$ & $-3.7671 E-04$ & $2.6490 \equiv+00$ & $1.8950 E-04$ & $-4.7078 E-06$ \\
\hline 90.0000 & $2.6781 E-02$ & $-2.9762 E-04$ & $2.6507 E+00$ & $1.4998 \mathrm{E}-04$ & $-3.3063 E-06$ \\
\hline 100.0000 & $2.4102 E-02$ & $-2.4106 E-04$ & $2.6521 \equiv+00$ & $1.2171 E-04$ & $-2.4102 E-06$ \\
\hline 000 & $1.2050 E-02$ & $-6.0254 E-05$ & $2.6582 E+00$ & $3.1330 E-05$ & $-3.0126 E-07$ \\
\hline 300.0000 & $8.0334 E-03$ & $-2.6779 E-05$ & $2.6504 E+00$ & $1.4594 E-05$ & $-8.9260 E-08$ \\
\hline 100.0000 & $6.0250 E-03$ & $-1.5053 E-05$ & $2.6615 \equiv+00$ & $8.7363 E-06$ & $-3.7657 E-08$ \\
\hline 000.0000 & $4.8200 E-03$ & $-9.6401 E-06$ & $2.6622 E+00$ & $6.0250 E-05$ & $-1.9280 E-08$ \\
\hline & $4.0167 E-03$ & $-6.6945 E-05$ & $2.6627 \geqq+00$ & $4.5522 E-06$ & $-1.1157 E-08$ \\
\hline 100.0000 & $3.4429 E-03$ & $-4 \cdot 9184 E-06$ & $2.6631 E+00$ & $3.6642 E-06$ & $-7.0263 E-09$ \\
\hline 100.0000 & $3.0125 E-03$ & $-3.7656 E-06$ & $2.6635 \equiv+00$ & $3.0878 E-06$ & $-4.7070 E-09$ \\
\hline 00.0000 & $2.6778 E-03$ & $-2.9753 E-06$ & $2.6638 E+00$ & $2.6927 E-06$ & $-3.3059 E-09$ \\
\hline 1000.0 & $2.4100 E-03$ & $-2.4100 E-06$ & $2.6540 \equiv+00$ & $2.4100 E-06$ & $-2.4100 E-09$ \\
\hline
\end{tabular}

5. $3518 E-03$ $2.1386 E-02$ $4.8037 E-02$ $8.5198 E-02$ $1.3272 E-01$ $1.9042 E-01$ 2.5806E-01 $3.3538 \mathrm{E}-01$ $4.2209 E-01$ $5.1785 E-01$ $1.8838 E+00$ $5.6594 E+00$ 7.0102E+00 $8.2343 E+00$ 9.1495E+0O $9.1495 E+00$ $9.8112 E+00$
$1.0280 E+01$ $1.0607 \mathrm{E}+01$ $1.1197 E+01$ $1.0989 E+01$ $1.0809 E+01$ $1.0678 E+01$ $1.0582 E+01$ $1.0508 E+01$ $1.0451 E+01$ $1.0405 E+01$ $1.0368 E+01$ $1.0189 E+01$ $1.0126 E+01$ $1.0094 E+01$ $1.0075 E+01$ $1.0062 E+01$ $1.0053 E+01$ $1.0046 E+01$ $1.0040 E+01$ $1.0036 E+01$ $1.0016 E+01$ $1.0009 E+01$ $1.0006 E+01$ $1.0003 E+01$ $1.0002 E+01$ $1.0001 E+01$ $1.0000 E+01$ $1.0000 E+01$
ZIA U

4.271 SE -02 $8.5287 E-02$ $1.2755 E-0$ $2.1058 E-0$ $2.5105 E-0$ $2.9065 E-01$ $3.2924 E-01$ $3.6669 E-01$ $4.0290 E-01$ $6.7643 E-01$ 6.6214E 01 $5.0060 E-01$ 2.7945E-01 $6.1852 E-02$ $-1.300 \mathrm{CE}-01$ $-2.8836 E-01$ $-4.1353 E-01$ $-4.1353 E-01$
$-7.1554 E-0$ $-6.1505 E-01$ $-5.1334 E-01$ $-4.3547 E-01$ $-3.7658 E-01$ $-3.3112 E-01$ $-2.9518 E-01$ $-2.6614 E-01$ $-2.4223 E-01$ $-1.2717 E-01$ $-8.6122 E-02$ $-6.5094 E-02$ $-5.2316 E-02$ $-4.3731 E-02$ -3.756 EE -02 $-3.2924 E-02$ $-2.9303 E-02$ $-2.6399 E-02$ $-1.3260 E-02$
$-8.8533 E-03$ $-8.8533 E-03$ $-6.6450 E-03$ $-4.4333 E-03$ $-4.4333 E-03$
$-3.8008 E-03$ $-3.3262 E-03$ $-2.9570 E-D 3$

$-2.6616 E-03$
2/A H

$8.5489 E+00$ $8.5456 E+00$
$8.5354 E+00$

$8.5186 E+00$

$8.4951 E+00$

$8.4650 E+00$

$8.4650 E+00$

$8.3855 E+00$

$8.3365 E+00$

$8.2814 E+00$

$8.2205 E+00$

$6.1708 E+00$

$6.1708 E+00$

$3.8706 \mathrm{E}+00$

$2.9768 \mathrm{E}+00$

$2.2786 E+00$

$1.7482 E+0$

$1.3504 E+0 D$

$1.0531 E+00$

$1.5495 E-01$

$4.5840 E-02$

$1.9195 \mathrm{E}-02$

$9.7759 E-03$

$3.5417 E-03$

$2.3688 E-03$

$1.6617 E-03$

$1.2103 E-03$

$1.5086 E-04$

$4.4693 E-05$

$1.8864 E-05$

$9.6660 E-06$

$5.5995 E-06$

$3.5307 E-06$

$2.3688 E-06$

$1.6664 E-06$

$1.2171 E-06$

$1.5665 E-07$

4.8647E-08

2.1841E-08

$7.5871 E-0$

$5.2346 E-09$

$3.8598 \mathrm{E}-09$

$2.9918 E-09$
$2.4100 E-09$
Z2/A DHDR $6.7650 E-01$ $-1.3505 E+00$ $-2.0197 E+00$ $-2.6815 E+00$ $-3.3336 E+00$ $-3.9739 E+00$ $-4.6000 E+00$ $-5.2100 E+00$ $-6.3738 E+00$ 1.0717E+01 - $2295 E+01$ $-1.1688 \mathrm{E}+0$ $-9.9495 E+00$ $-7.9291 \mathrm{E}+0 \mathrm{O}$ $-6.0857 E+00$ $-4.5826 E+00$ $-2.2994 E-01$ $-1.4520 E-02$ $-5.9074 E-03$ $-2.8349 E-03$ $-1.5248 E-03$ $-8.9158 \mathrm{E}-04$ $-5.5558 E-0$ $-3.6401 E-04$ $-2.2640 E-05$ $-4.4682 E-06$ $-8.8456 E-08$ $-3.6273 E-08$ $-3.6273 E-08$ $-4.5969 E-10$ $-1.4874 E-10$ $-6.266 G E-11$ $-3.1241 E-11$ $-1.0709 E-11$

$-6.9975 E-12$ 


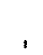




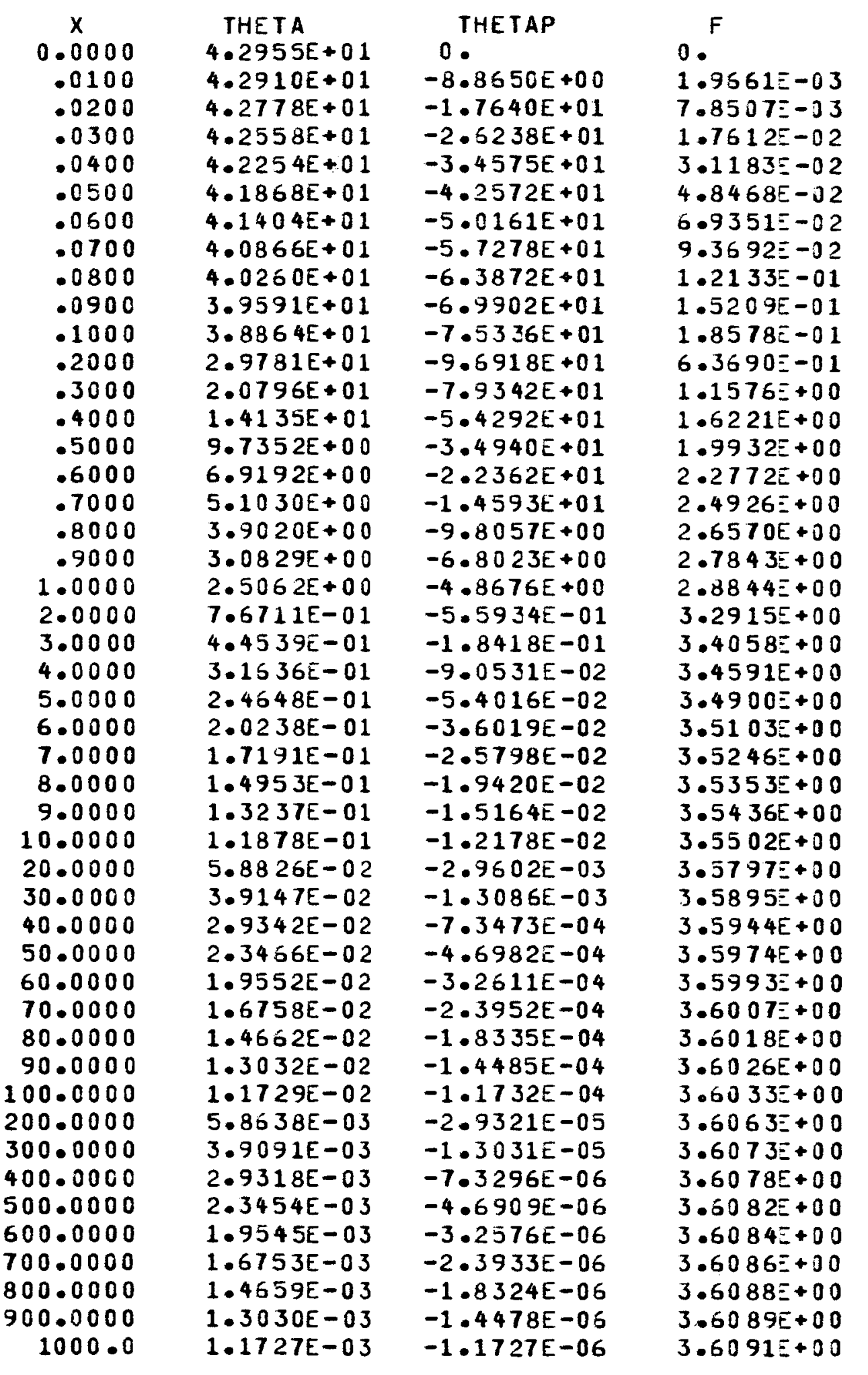

FP

3.9299E-01 7. $3322 E-01$ $1.5446 E+00$ $1.91065+00$ $1.2637 \mathrm{E}+00$ $2.5018 E+00$ $2.9231 E+00$ $2.9231 E+00$ $3.5090 E+00$ $5.1556 E+00$ $5.0490 \mathrm{E}+00$ $4.1892 E+00$ $3.2477 E+00$ $2.4650 E+00$ $1.8728 E+00$ $1.4390 E+00$ $1.1228 E+00$ 8.9079E-01 $1.7981 E-01$ $7.2625 E-02$
$3.9140 E-02$ $3.9140 E-02$ $2.4504 E-02$ $1.6802 E-02$ $1.2248 E-02$ $9.3281 E-03$ . .9327E-03 (.4TEE-OS (3.5296E-04 $2.3524 E-04$ . $9.2222 E-05$ .2987E-05 5. $9229 E-05$ $1.5245 E-05$ $7.1015 E-06$ $4.2511 E-06$ 2.9318E-06 $2.2151 E-06$ $1.7830 E-06$ $1.5025 E-06$ $1.3103 E-06$
$1.1727 E-06$
FPP $3.9346 E+01$ $3.9207 E+0$ $3.8110 E+01$ $3.7172 E+01$ $3.7172 E+01$ $3.4594 E+01$ $3.2998 E+01$ $3.1229 E+01$ $2.9315 E+01$ $2.7283 E+O$ $2.7283 E+01$ $-6.3957 E+0 D$ $-9.6930 E+00$ $-8.7798 E+00$ $-6.8446 E+00$ $-5.0599 E+00$ $-3.6865 E+03$ $-2.6931 E+00$ $-1.9884 E+00$ $-2.0575 E-01$ -5.28JJE-02 $-1.0199 E-02$ -3.5768E-OS $-2.3722 E-03$ $-1.1999 E-03$ $-1.4746 E-0$ $-1.8351 E-05$ $-5.4328 E-06$ $-3.4207 E-06$ $-2.2913 E-06$ $-1.5091 E-05$ $-1.1730 E-06$ $-1.4650 E-07$ $-4.3435 E-08$ $-4.3435 E-08$
$-1.8324 E-08$ $-9.3818 E-09$ $-5.4293 E-09$ $-3.4190 E-09$ $-2.2905 E-09$ $-1.6087 E-09$ -1.1727 E-09 $s$

8.6556E-0 2 $3.4507 E-01$ . $107 \mathrm{E}+\mathrm{O}$ $2.9982 E+00$ $4.0239 E+00$ $5.1720 \mathrm{E}+00$ $6.4292 E+00$ $7.7815 E+00$ $2.3512 E+01$ $2.3512 E+01$ $4.4526 \mathrm{E}+01$ $4.8575 E+01$ $5.0536 E+01$ $5.1450 E+01$ $5.1851 \mathrm{E}+01$ $5.2001 E+01$ $5.2025 E+01$ $5.1350 \mathrm{E}+01$ $5.0943 E+01$ $5.0721 \mathrm{E}+01$ $5.0583 E+01$ $5.0489 E+01$ $5.0421 E+01$ $5.0369 E+01$ $5.0329 E+01$ $5.0296 E+01$ $5.0148 E+01$ $5.0098 E+01$ $5.0058 E+01$ $5.0048 E+01$ $5.0041 E+01$ $5.0035 E+01$ $5.0031 \mathrm{E}+01$ $5.0027 \mathrm{E}+01$ $5.0012 \mathrm{E}+01$ $5.0007 E+01$ $5.0005 E+01$ $5.0003 E+01$ $5.0002 E+01$ $5.0001 \mathrm{E}+01$ $5.0001 E+01$

$5.0000 E+01$

$5.0000 E+01$
Z/A U

$1.9638 E-01$ 3.9065E-01 $5.8088 E-01$ $9.4122 E-01$ $1.1078 E+00$ $1.2634 E+00$ $1.4064 \mathrm{E}+00$ $1.5360 E+00$ $1.536 E+00$ $1.9711 \mathrm{E}+00$ $1.1905 E+00$ $1.3380 E-0$ $-7.3865 E-01$ $-1.3303 E+00$ $-1.6880 E+00$ $-1.8823 E+0$ $-1.9708 E+00$ $1.9936 E+00$ - $1.4659 E+00$ $-1.0626 E+00$ $-8.2562 E-01$ $-6.7350 E-01$ $-4.9127 E-01$ - 3.863 E 01 - $1.7752 E-01$ -1.1900E-O $-8.9493 E-02$ T.1712E-O $-5.9825 E-02$ -5.131 SE -02 $-3.9956 E-0$ $-3.5973 E-02$ $-1.801 \in E-02$ $-1.801 \epsilon E-02$
$-1.2017 E-02$ $-9.0153 E-03$ $-7.2135 E-03$ $-6.0119 E-03$ $-5.1534 E-03$ $-4.5095 E-03$ $-4.008 E E-03$
$-3.6079 E-03$
Z/A H $3.9346 E+01$ $3.9299 E+01$ $3.9161 E+01$ $3.8614 E+01$ $3.8212 E+01$ $3.7728 E+01$ $3.7169 E+0$ $3.5538 E+01$ $3.5843 E+01$ $3.5090 \mathrm{E}+01$ $3.5090 E+0$ $2.5778 E+01$
$1.6830 E+01$ $1.6830 E+01$ $6.4954 E+00$ $4.1083 E+00$ $1.7988 E+00$ $1.2476 E+00$ $8.9079 E-01$ $8.9905 E-02$ $2.4208 E-02$ $9.7849 E-03$ 4.9007E-O $2.8004 E-03$ $1.7496 \mathrm{E}-03$ $1.1660 E-03$ $8.1595 E-04$ $5.9327 E-04$ 7.3542E 05 $2.1765 E-05$ $9.1833 E-06$ $2.7253 E-06$ $1.7183 E-06$

$1.1528 E-06$ $8.1096 E-07$ $5.9229 E-07$ $7.6229 \mathrm{E}-08$ $2.3672 E-08$ $2.3672 E-08$ $5.8636 E-09$ $3.6919 E-09$ $2.5472 E-09$ $1.8782 E-09$ $1.4558 E-09$
Z2/A DHDR $-9.2570 E+00$ $-1.8416 E+01$
$-2.7381 E+01$ $-3.6061 E+01$ $-4.4372 E+01$ $-5.2236 E+01$ $-6.6370 E+01$ $-7.7423 E+01$ $-5.0415 E+01$ $-3.0550 E+01$ $-1.8255 E+01$ $-1.1051 E+01$ $-6.8565 E+00$ $-4.3785 E+00$ $-2.8792 E+00$ $-1.4783 E-01$ $-2.5681 E-02$ $-3.0200 E-03$ $-1.4276 E-03$ $-7.6091 E-04$ $-4.4228 E-04$ $-1.7931 E-04$ $-1.1050 E-05$ $-2.1771 E-06$ $-6.8836 E-07$ $-2.81915-07$ $-7.3414 E-08$ $-4.3051 E-08$ $-2.6890 E-08$ $-3.0491 E-11$ $-1.5202 E-11$ $-8.5231 E-12$ $-5.2108 \mathrm{E}-1$ $-2.3454 E-12$ 
$$
\text { " }
$$ 


$\begin{array}{rc}x & \text { THETA } \\ C .0000 & 8.0541 E+01 \\ .0100 & 8.0383 E+01 \\ .0200 & 7.9911 E+01 \\ .0300 & 7.9134 E+01 \\ .0400 & 7.8066 E+01 \\ .0500 & 7.6726 E+01 \\ .0500 & 7.5136 E+01 \\ .0700 & 7.3324 E+01 \\ .0800 & 7.1317 E+01 \\ .0900 & 6.9145 E+01 \\ .1000 & 6.5840 E+01 \\ .2000 & 4.2135 E+01 \\ .3000 & 2.4006 E+01 \\ .4000 & 1.3837 E+01 \\ .5000 & 8.4378 E+00 \\ .6000 & 5.4545 E+00 \\ .7000 & 3.8047 E+00 \\ .8000 & 2.7784 E+00 \\ .9000 & 2.1214 E+00 \\ 1.0000 & 1.5806 E+00 \\ 2.0000 & 4.7322 E-01 \\ 3.0000 & 2.6843 E-01 \\ 4.0000 & 1.8959 E-01 \\ 5.0000 & 1.4733 E-01 \\ 6.0000 & 1.2081 E-01 \\ 7.0000 & 1.0254 E-01 \\ 8.0000 & 8.9144 E-02 \\ 9.0000 & 7.8888 E-02 \\ 10.0000 & 7.0772 E-02 \\ 20.0000 & 3.5023 E-02 \\ 30.0000 & 2.3304 E-02 \\ 40.0000 & 1.7466 E-02 \\ 50.0000 & 1.3969 E-02 \\ 60.0000 & 1.1539 E-02 \\ 70.0000 & 9.9749 E-03 \\ 80.0000 & 8.7274 E-03 \\ 90.0000 & 7.7573 E-03 \\ 100.0000 & 5.9814 E-03 \\ 200.0000 & 3.4903 E-03 \\ 300.0000 & 2.3268 E-03 \\ 400.0000 & 1.7451 E-03 \\ 500.0000 & 1.3961 E-03 \\ 500.0000 & 1.1634 E-03 \\ 700.0000 & 9.9720 E-04 \\ 800.0000 & 8.7255 E-04 \\ 900.0000 & 7.7560 E-04 \\ 1000.0 & 6.9804 E-04 \\ & \end{array}$

THETAP

0. $-6.2646 E+01$
$-9.2533 E+01$ $-1.2076 E+02$ $-1.4689 E+02$ $-1.7055 E+02$ $-1.9145 E+C$ $-2.0942 E+02$ $-2.2437 E+02$ $-2.3528 E+02$ $-2.2661 E+0$ $-1.3654 E+02$ $-7.2829 E+01$ $-3.8963 E+01$ $-2.1814 E+0$ $-1.2916 E+01$
$-8.0768 E+00$ $-8.0768 E+00$ $-5.3074 E+00$ $-3.5739 E-01$ $-3.5739 E-01$ $-5.4931 E-02$ $-3.2550 E-02$ $-1.5452 E-02$ $-1.1614 E-02$ $-1.1614 E-02$
$-9.0596 E-03$ $-9.0596 E-03$ $-1.7633 E-03$ $-1.7633 E-03$
$-7.7920 E-04$ $-4.3741 E-04$ $-2.7968 E-04$ $-1.9413 E-04$ $-1.4258 E-04$ $-1.0914 E-04$ $-8.6222 \varepsilon-05$ $-6.9833 E-05$ $-1.7453 E-05$ $-7.7564 E-06$ $-4.3629 E-06$ $-2.7922 E-06$ $-1.9390 \mathrm{E}-06$ $-1.4246 E-06$ $-1.0907 E-06$
$-8.0178 E-07$ $-8 \cdot 6178 E-07$
$-6.9804 E-07$
0. $3.8334 \Sigma-03$ $1.5285 \equiv-02$ $3.4211 \equiv-02$ $0.0377=-02$ $1.3308=-01$ $1.7877 E-0$ $2.3002=-01$ $2.3630 \equiv-01$ $3.4703 E-0$ $1.0787 E+00$ $1.7698 E+00$ $2.2821 \equiv+00$ $2.5351 \equiv+00$ $2.8796 E+00$ $3.0506=+00$ $3.1740=+00$ $3.2555=+00$ $3.6004=00$ $3.67045+00$ $3.7025=+00$ $3.7211=+00$ $3.7333=+00$ $3.7418 \bar{E}+00$ $3.7482 E+00$ $3.7531 \equiv+00$ $3.7571 \equiv+00$ $3.7747 \bar{E}+00$ $3.7805 \equiv+00$ $3.7834 \equiv+00$ $3.7852 E+00$ $3.7863 E+00$ $3.7872 E+00$ $3.7878=+00$ $3.7883=+00$ $3.7887 E+00$ $3.7905 \equiv+00$ $3.7911 E+00$ $3.7914 E+00$ $3.7916 E+30$ $3.7918 E+00$ $3.7919 E+00$ $3.7920=+00$ $3.7921 \equiv+00$
$F$ $1.5221 E+00$ $2.2592 \mathrm{E}+00$ $3.5422 E+00$ $4.2732 E+00$ $4.8559 E+00$ $5.3859 E+00$ 3.8601E+00 $5.2767 E+00$ $7.5810 \mathrm{E}+00$ $6.0507 E+00$ $4.2508 E+00$ $2 \cdot 9127 E+00$ $2.0215 E+00$ $1.4391 E+00$ $1.0538 E+00$ 7. $9298 E-01$ 1.1 $140 E-01$ $4.4006 E-02$ $1.4006-02$ $1.4676 \mathrm{E}-02$ $1.0675 E-02$

7. $3128 E-03$ $5.5655 \mathrm{E}-03$ $5.5655 E-03$
$4.3792 E-03$ $3.5366 E-03$ $8.7581 E-04$ $3.8873 E-04$ $2.1867 E-04$ $1.4003 E-04$ $9.7335 E-05$ $7.1597 E-05$ $5.4895 E-05$ $4.3445 E-05$ 3. $5256 E-05$ $9.0748 E-06$ $4.2271 E-06$ $2.5304 E-06$ $1.7451 \mathrm{E}-06$ $1.3185 \mathrm{E}-06$ $1.0613 E-06$ $8.7437 E-07$ $5.9804 E-07$
FPP

$7.6749 E+01$ $7.6263 E+01$ $7.4821 E+01$ $7.2467 E+O$
$6.9274 E+0$ $6.5337 E+0$ $6.0769 E+01$ $5.5696 E+01$ $5.0249 E+01$ $4.4558 E+01$ $3.3752 E+01$
$-7.1311 E+00$ $-1.9075 E+01$ $-1.5952 E+C 1$ $-1.0925 E+01$ $-7.1465 E+00$ $-4.6880 E+00$ $-3.1367 E+0$ $-2.1523 E+0$ $-1.5155 E+00$ $-3.2545 E-02$ $-1.2579 E-02$ $-6.1467 E-03$ $-3.4612 E-03$ $-2.1423 E-0$
$-1.4187 E-0$ $-1.4187 E-03$
$-9.8841 E-04$ $-9.8841 E-04$
$-7.1634 E-04$ $-8.7835 E-05$ $-2.5930 E-05$ $-1.0925 E-05$ $-5.5903 E-06$ $-3.2341 E-0$ $-2.0362 E-0$ $-1.3639 E-05$ $-1.3639 E-05$
$-9.5785 E-07$
$-6.9823 E-07$
$-8.7261 E-08$ $-8.7251 E-0$ $-8.7261 E-0.3$
$-2.5854 E-09$ $-1.0907 E-08$ $-5.5844 E-0$ $-3.2317 E-0$ $-2.0351 E-0$ $-1.3634 E-0$ $-9.5754 E-10$
$-6.9804 E-10$
3.1245E-01 $1.2422 E+00$ $2.7666 E+00$ $4.8494 E+00$ $7.4422 E+00$ $1.0487 E+01$ $1.3919 E+01$ $1.7669 E+01$ $2.1665 E+01$ $2.5838 E+01$ $5.5578 E+0$ $8.7826 E+01$ 9.6819E+01 $1.0013 E+02$ $1.0131 E+02$ $1.0169 E+02$ $1.0177 E+02$ $1.0173 E+02$ $1.0093 \mathrm{E}+02$ $1.0093 E+02$ $10047 E+02$ $.0038 E+02$ $1.0027 E+02$ $1.0024 E+02$ $1.0021 E+02$ $1.0019 E+02$ $1.0009 E+02$ $1.0006 E+02$ $1.0005 E+02$ $1.0004 E+02$ $1.0003 E+02$ $1.0003 E+02$ $1.0002 E+02$ $1.0002 E+02$ $1.0002 E+02$ $1.0001 E+02$ $1.0000 E+02$ $1.0000 E+02$ $1.0000 E+02$ $1.0000 E+02$ $1.0000 E+02$ $1.0000 E+02$
$1.0000 E+02$
.8253E-0 1 $.5782 E-01$ $1.459 .2 E+00$ $1.7730 E+00$ $2.0552 E+00$ $2.3021 E+00$ $2.51 G E E+00$ $2.6790 E+00$ $2.8064 E+00$ $2.1877 E+00$ $1.5127 E-01$ $-1.4544 E+00$ $-2.3594 E+00$ $-2.9190 E+00$ $-2.919 \mathrm{OE}+00$ 2.035 $3 E+00$ $-2.7234 E+00$ $-1.6888 E+00$ $-1.1795 E+00$ $-9.0211 E-01$ $-7.2955 E-01$ $-601217 E-01$ $-5.2723 E-01$ $-4.6296 E-01$ $-4.1264 E-01$ $-3.7217 E-01$ $-1.8786 E-01$ $-1.2563 \mathrm{E}=01$ $-9.4367 E-02$ $-7.5563 E-02$ $-6.3008 E-02$ $-5.4031 E-02$ $-4.7293 E-02$ $-4.2049 E-02$ $-3.7851 E-02$ $-1.8943 E-0$ $-1.263 J E-02$ $-9.4760 E-03$ $-6.3183 E-03$ $-5.4159 E-03$ $-4.7391 E-03$ $-4.212 \in E-03$

$21 A W$
$7.6749 E+0$ $7.6587 \mathrm{E}+01$ $7.6104 E+01$ $7.5308 E+01$ $7.4215 E+01$ $7.2845 E+01$ $7.1221 E+01$ 列 $6.2767 E+01$ $3.7905 E+01$ $2.0169 E+01$ $1.0627 E+01$ $5.8254 E+0 O$ $3.3692 E+00$ $2.0558 E+00$ $1.3172 E+00$ $6.1184 E-01$ $1.569 E-02$ $5.8821 E-03$ 


\section{APPENDIX B}

TABULATED NUMERICAL RESULTS FOR A POINT SOURCE IN AN INFINITE MEDIUM

The numerical results tabulated in this appendix are solutions to Equations (33), (34), and (35). Reading from left to right, the parameters tabulated are: $n, f(n), f^{\prime}(n), \theta(n), \theta^{\prime}(n)$, and $S(n)$. The parameter $S(n)$ is defined in Equation (33). When $n=1, S(n)$ is equal to the Rayleigh number for the solution. Results are tabulated for Rayleigh numbers of $0.001,0.01,0.1,1.0,5.0,10$, 50, and 100. The parameter $\mathrm{C}$ is the constant of integration $\mathrm{C}_{2}$. 
$C=.41667 E-07$

ETA

$-1.0000$

$-.9000$

$-.8000$

$-.7000$

$-.6000$

$-.5000$

$-.4000$

$-.3000$

$-.2000$

$-.1000$

$-.0500$

0.0000

.1000

.2000

.3000

.4000

.5000

.6000

.7000

.8000

.9000

1.0000
$F$

0.

47493E-04 $.89988 E-04$ $12749 E-03$

$15998 \mathrm{E}-03$ $.18748 \mathrm{E}-03$ . $20999 E-03$ .22749E-03 . 23999E-03 24750E-03 $.24937 \mathrm{E}-03$ . $25000 \mathrm{E}-03$ $24750 \mathrm{E}-03$ $.24001 E-03$ . $22751 \mathrm{E}-03$ $.21001 E-03$ $18752 E-03$ $16002 E-03$ . $12751 \mathrm{E}-03$ $.90012 E-04$ 0 .
FP

$.49990 E-03$ $44994 E-03$ 39996E-03 $34998 E-03$ 30000E-03 .25001E-03 . $20002 E-03$ $15003 E-03$ $.10004 E-03$ $50040 E-04$ $.25041 \mathrm{E}-04$ $41667 E-07$ $-.49960 \mathrm{E}-04$ $-.99963 E-04$ $-.14997 E-03$ $-.19998 \mathrm{E}-03$ $-.24999 \mathrm{E}-03$

$-.30000 \mathrm{E}-03$

$-.35002 E-03$

$-.40004 \mathrm{E}-03$

$-.45006 \mathrm{E}-03$

$-.50008 E-03$
THETA

$.49985 E-03$

49989E-03

.49990E-03

. $49991 \mathrm{E}-03$

49992E-03

49994E-03

$.49995 E-03$

49996E-03

$.49997 E-03$

49999E-03

49999E-03

$50000 \mathrm{E}-03$

$50001 E-03$

.50002E-03

$50004 E-03$

$.50005 E-03$

$.50006 E-03$

$50007 E-03$

50009E-03

$50010 E-03$

$.50011 \mathrm{E}-03$

$50013 E-03$
THETAP

$12494 E-06$

$12495 E-06$

$12496 E-06$

$12496 \mathrm{E}-06$

$12497 \mathrm{E}-06$

$12497 \mathrm{E}-06$

$12498 E-06$

$12498 \mathrm{E}-06$

$12499 E-06$

12499E-06

$12500 E-06$

$12500 \mathrm{E}-06$

$12501 \mathrm{E}-06$

$.12501 \mathrm{E}-06$

- $12502 E-06$

$.12502 E-06$

$.12503 \mathrm{E}-06$

. $12503 \mathrm{E}-06$

. $12504 \mathrm{E}-06$

$.12504 E-06$

$.12505 E-06$

$.12505 E-06$
RA

0.

49964E-04

$.99933 E-04$ . 14990E-03

.19988E-03

$.24986 E-03$

$.29984 E-03$

$.34983 \mathrm{E}-03$

$.39982 \mathrm{E}-03$

$.44981 E-03$

$.47481 E-03$

$.49981 E-03$

$.54981 E-03$

$.59982 E-03$

$.64983 E-03$

$.69984 E-03$

$.74986 E-03$

$.79988 \mathrm{E}-03$

$.84990 E-03$

$.89993 E-03$

$.94996 E-03$

$.10000 E-02$ 
$C=\quad .41666 E-05$

ETA

$-1.0000$

-.9459

$-.9000$

$-.8000$

$-.7000$

$-.6000$

$-.5000$

$-.4000$

$-.3000$

$-.2000$

$-.1500$

$-.1000$

$-.0500$

0.0000

.0500

.1000

.1500

.2000

.3000

.4000

.5000

.6000

.7000

.8000

.9000

1.0000
$F$

0.

.26273E-03

$.47429 \mathrm{E}-03$

$.89880 \mathrm{E}-03$

$.12735 E-02$

$.15984 E-02$

$18734 E-02$

. 20986E-02

. 22739E-02

. 23992E- 02

.24431E-02

.24746E-02

24935E-02

.25000E-02

. 24939E-02

$.24754 E-02$

- $24444 E-02$

24008E-02

. $22761 E-02$

$21014 E-02$

$.18766 E-02$

. $16016 E-02$

$12765 E-02$

$90120 E-03$
FP

$.49915 \mathrm{E}-02$

$.47225 E-02$

$.44940 E-02$

$.39962 E-02$

$.34980 E-02$

. 29997E-02

$25010 E-02$

20022E-02

$15030 E-02$

$10037 E-02$

$.75388 \mathrm{E}-03$

.50404E-03

$25413 E-03$

$41666 \mathrm{E}-05$

$-.24586 E-03$

$-.49596 \mathrm{E}-03$

$-.74611 E-03$

$-.99633 E-03$

$-.14970 E-02$

$-.19978 E-02$

$-.24989 E-02$

$-.30003 E-02$

$-.35019 E-02$

$-.40038 E-02$

$-.45060 \mathrm{E}-02$

$-.50083 E-02$
THETA

$.49873 E-02$ 49882E-02 49887E-02 $.49900 E-02$ $.49912 E-02$ 49925E-0Z 49937E-02 49950E-02

49962E-02

49975E-02

49981E-02

49987E- 02

49993E-02

$50000 E-02$

50006E-02

$50012 E-02$

$50018 \mathrm{E}-02$

$50025 E-02$

$50037 E-02$

$50050 E-02$

$50062 E-02$

50075E-02

50087E-02

$50100 E-02$

$50112 E-02$

$.50125 E-02$
THETAP

$12447 E-04$ $.12451 E-04$

$.12453 \mathrm{E}-04$

$.12458 E-04$

$12464 E-04$

$.12469 E-04$

. $12474 E-04$

$.12479 \mathrm{E}-04$

. $12484 E-04$

$.12489 E-04$

. $12492 \mathrm{E}-04$

$.12495 E-04$

$.12497 E-04$

. $12500 E-04$

$.12502 E-04$

$.12505 E-04$

. $12508 E-04$

$12510 E-04$

- $12516 E-04$

$12521 E-04$

$.12526 E-04$

$.12531 E-04$

$12536 E-04$

$.12542 E-04$

. $12547 \mathrm{E}-04$

$.12552 E-04$
RA

0 .

26849E-03

$.49645 E-03$

$.99326 E-03$

$14905 E-02$

$.19880 E-02$

. 24860E-02

$.29843 E-02$

$.34830 E-02$

39820E-02

$.42317 E-02$

$.44814 E-02$

$.47313 E-02$

$.49813 E-02$

$.52313 E-02$

$.54814 E-02$

$.57317 E-02$

59820E-02

$.64829 E-02$

$69842 E-02$

$.74859 E-02$

$.79880 E-02$

$.84904 E-02$

$.89932 E-02$

$.94964 E-02$

$.10000 E-01$ 
$C=\quad .41638 E-03$

\begin{tabular}{|c|c|c|c|c|c|}
\hline ETA & $F$ & FP & THETA & THETAP & RA \\
\hline $\begin{array}{l}-1.0000 \\
-.9459 \\
-.9000 \\
-.8000 \\
-.7000 \\
-.6000 \\
-.5000 \\
-.4000 \\
-.3000 \\
-.2000 \\
-.1500 \\
-.1000 \\
-.0500 \\
0.0000 \\
.0500 \\
.1000 \\
.1500 \\
.2000 \\
.3000 \\
.4000 \\
.5000 \\
.6000 \\
.7000 \\
.8000 \\
.9000 \\
1.0000\end{array}$ & $\begin{array}{r}0 . \\
.25897 E-02 \\
.46782 E-02 \\
.88786 E-02 \\
.12599 E-\theta 1 \\
.15836 E-01 \\
.18589 E-01 \\
.20854 E-01 \\
.22629 E-01 \\
.23912 E-01 \\
.24369 E-01 \\
.24701 E-01 \\
.24909 E-01 \\
.24992 E-01 \\
.24950 E-01 \\
.24783 E-\theta 1 \\
.24491 E-01 \\
.24072 E-01 \\
.22857 E-01 \\
.21134 E-01 \\
.189011 E-01 \\
.16156 E-01 \\
.12896 E-01 \\
.91185 E-02 \\
.48207 E-02 \\
-.34694 E-17\end{array}$ & $\begin{array}{r}.49163 \mathrm{E}-01 \\
.46588 \mathrm{E}-01 \\
.44397 \mathrm{E}-01 \\
.39607 \mathrm{E}-01 \\
.34793 \mathrm{E}-01 \\
.29955 \mathrm{E}-01 \\
.25093 \mathrm{E}-01 \\
.20206 \mathrm{\theta}-01 \\
.15296 \mathrm{E}-01 \\
.10361 \mathrm{E}-01 \\
.78839 \mathrm{E}-02 \\
.54010 \mathrm{E}-02 \\
.29118 \mathrm{E}-02 \\
.41638 \mathrm{E}-03 \\
-.20853 \mathrm{E}-02 \\
-.45932 \mathrm{E}-02 \\
-.71074 \mathrm{E}-02 \\
-.96279 \mathrm{E}-02 \\
-.14688 \mathrm{E}-01 \\
-.19773 \mathrm{E}-01 \\
-.24884 \mathrm{E}-01 \\
-.30021 \mathrm{E}-01 \\
-.35184 \mathrm{E}-01 \\
-.40373 \mathrm{E}-01 \\
-.45588 \mathrm{E}-01 \\
-.50829 \mathrm{E}-01\end{array}$ & $\begin{array}{l}.48746 \mathrm{E}-\theta 1 \\
.48812 \mathrm{E}-\theta 1 \\
.48867 \mathrm{E}-01 \\
.48988 \mathrm{E}-01 \\
.49109 \mathrm{E}-01 \\
.49230 \mathrm{E}-01 \\
.49353 \mathrm{E}-01 \\
.49475 \mathrm{E}-01 \\
.49598 \mathrm{E}-01 \\
.49722 \mathrm{E}-01 \\
.49784 \mathrm{E}-01 \\
.49846 \mathrm{E}-01 \\
.49908 \mathrm{E}-01 \\
.49971 \mathrm{E}-01 \\
.50033 \mathrm{E}-01 \\
.50096 \mathrm{E}-01 \\
.50158 \mathrm{E}-01 \\
.50221 \mathrm{E}-01 \\
.50348 \mathrm{E}-01 \\
.50474 \mathrm{E}-01 \\
.50602 \mathrm{E}-01 \\
.50729 \mathrm{E}-01 \\
.50858 \mathrm{E}-01 \\
.50986 \mathrm{E}-01 \\
.51116 \mathrm{E}-01 \\
.51246 \mathrm{E}-01\end{array}$ & $\begin{array}{l}.11983 E-02 \\
.12010 \mathrm{E}-\theta 2 \\
.12032 \mathrm{E}-02 \\
.12082 \mathrm{E}-02 \\
.12132 \mathrm{E}-02 \\
112182 \mathrm{E}-02 \\
.12232 \mathrm{E}-\theta 2 \\
.12283 \mathrm{E}-02 \\
.12334 \mathrm{E}-02 \\
.12385 \mathrm{E}-02 \\
.12411 \mathrm{E}-02 \\
.12437 \mathrm{E}-02 \\
.12463 \mathrm{E}-02 \\
.12489 \mathrm{E}-02 \\
.12515 \mathrm{E}-02 \\
.12541 \mathrm{E}-02 \\
.12567 \mathrm{E}-02 \\
.12593 \mathrm{E}-02 \\
.12646 \mathrm{E}-02 \\
.12699 \mathrm{E}-02 \\
.12752 \mathrm{E}-02 \\
.12806 \mathrm{E}-02 \\
.12860 \mathrm{E}-02 \\
.12914 \mathrm{E}-02 \\
.12969 \mathrm{E}-02 \\
.13024 \mathrm{E}-02\end{array}$ & $\begin{array}{r}0 . \\
.25122 E-02 \\
.46524 E-02 \\
.93396 E-02 \\
.14062 E-01 \\
.18820 E-01 \\
.23613 E-01 \\
.28443 E-01 \\
.33308 E-01 \\
.38211 E-01 \\
.40676 E-01 \\
.43150 E-01 \\
.45633 E-01 \\
.48126 E-01 \\
.50628 E-01 \\
.53140 E-01 \\
.55661 E-01 \\
.58191 E-01 \\
.63281 E-01 \\
.68409 E-01 \\
.73576 E-01 \\
.78781 E-01 \\
.84026 E-01 \\
.89311 E-01 \\
.94635 E-01 \\
.10000 E+00\end{array}$ \\
\hline
\end{tabular}




\begin{tabular}{|c|c|c|}
\hline ETA & $F$ & FP \\
\hline $\begin{array}{l}-1.0000 \\
-.9459 \\
-.9000 \\
-.8000 \\
-.7000 \\
-.6000 \\
-.5000 \\
-.4000 \\
-.3000 \\
-.2000 \\
-.1500 \\
-.1000 \\
. .0500 \\
0.0000 \\
.0500 \\
.1000 \\
.1500 \\
.2000 \\
.2500 \\
.3000 \\
.4000 \\
.5000 \\
.6000 \\
.7000 \\
.8000 \\
.9000 \\
1.0000\end{array}$ & 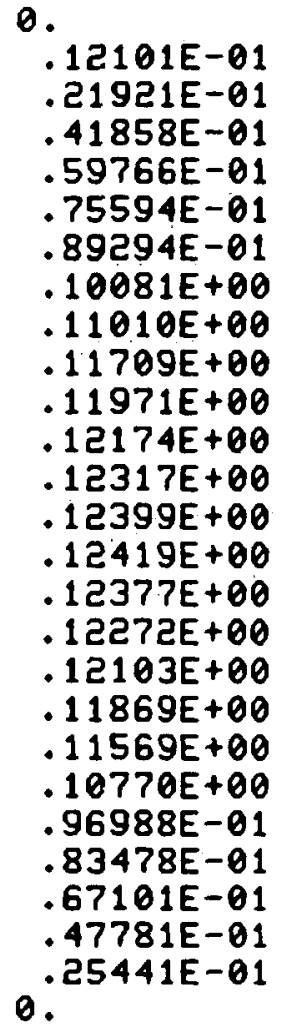 & $\begin{array}{r}.22896 \mathrm{E}+00 \\
.21842 \mathrm{E}+00 \\
.20937 \mathrm{E}+00 \\
.18931 \mathrm{E}+00 \\
.16876 \mathrm{E}+00 \\
.14772 \mathrm{\theta}+00 \\
.12618 \mathrm{E}+00 \\
.10411 \mathrm{E}+00 \\
.81494 \mathrm{E}-01 \\
.58324 \mathrm{E}-01 \\
.46525 \mathrm{E}-01 \\
.34580 \mathrm{E}-01 \\
.22487 \mathrm{E}-01 \\
.10244 \mathrm{E}-01 \\
-.21528 \mathrm{E}-02 \\
-.14704 \mathrm{E}-01 \\
-.27413 \mathrm{E}-01 \\
-.40282 \mathrm{E}-01 \\
-.53314 \mathrm{E}-01 \\
-.66511 \mathrm{E}-01 \\
-.93411 \mathrm{E}-01 \\
-.12100 \mathrm{E}+00 \\
-.14931 \mathrm{E}+00 \\
-.17836 \mathrm{E}+00 \\
-.20817 \mathrm{E}+00 \\
-.23877 \mathrm{E}+00 \\
-.27018 \mathrm{E}+00\end{array}$ \\
\hline
\end{tabular}

THETA

$.21872 E+00$ . $22008 \mathrm{E}+00$ . $22125 E+00$ . $22383 E+00$ . $22646 E+00$ . $22914 E+00$ $.23187 E+00$ . $23466 \mathrm{E}+00$ $.23750 E+00$ $.24040 \mathrm{E}+00$ $.24188 E+\theta 0$ $.24337 E+00$ $.24487 E+00$ $.24639 E+00$ $.24793 E+00$ $.24948 E+\theta 0$ $.25104 E+00$ . $25263 E+00$ $.25423 E+00$ . $25585 E+00$ . $25914 E+00$ $.26249 E+00$ $.26592 E+00$ . $26943 E+80$ $.27302 E+\theta 0$ - $27668 \mathrm{E}+00$ $.28043 E+00$
THETAP

$.25040 E-01$ . 25301E-01 . 25526E-01 $.26025 E-01$ . 26538E-01 - 27065E-01 - 27606E-01 .28162E-01 $.28734 E-01$ .29322E-01 - 29623E-01 $.29927 E-01$ $.30236 E-01$

$.30550 \mathrm{E}-01$

$.30867 E-01$

$31190 E-01$

$.31517 E-01$

$.31849 E-01$

$32186 E-01$

. 32527E-01

33226E-01

$.33945 E-01$

$.34686 \mathrm{E}-01$

$.35449 E-01$

. 36236E-01

$.37047 \mathrm{E}-01$

$.37883 \mathrm{E}-01$
RA

0 .

$.92129 E-02$

. $17176 E-01$

$.34994 E-01$

$.53476 \mathrm{E}-01$

. 72650E-01

$.92543 E-01$

$11318 \mathrm{E}+00$

$.13460 E+80$

$.15682 E+\theta \theta$

$.16825 E+0 \theta$

$.17989 E+00$

$19174 E+\theta 0$

$.20382 E+\theta 0$

. $21613 E+\theta \theta$

. $22867 E+00$

$.24145 E+08$

$.25447 E+00$

$.26773 E+00$

$.28125 E+00$

$30905 E+06$

$33793 E+00$

$.36792 E+08$

$.39907 E+0 \theta$

$.43143 E+\theta \theta$

$.46506 E+\theta \theta$

$.50000 E+00$ 
$C=.39074 E-01$

ETA

$-1.0000$

$-.9459$

$-.9000$

$-.8000$

$-.7000$

$-.6000$

$-.5000$

$-.4000$

$-.3000$

$-.2000$

$-.1500$

$-.1000$

$-.0500$

0.0000

.0500

.1000

.1500

.2000

.2500

.3000

.4000

.5000

.6000

.7000

.8000

.9000

1.0000
0.

$.22105 E-01$

40165E-01

$.77205 E-01$

$.11098 E+00$

$.14134 E+\theta 0$

$16813 E+\theta 0$

$19118 \mathrm{E}+00$

$.21032 E+00$

. $22536 E+00$

$.23128 E+\theta \theta$

$.23610 E+0 \theta$

$23980 E+\theta \theta$

$.24234 E+00$

$.24369 E+00$

$24384 E+00$

$.24275 E+00$

. $24038 \mathrm{E}+00$

. $23671 \mathrm{E}+00$

. $23170 E+00$

2 $1754 E+\theta \theta$

$.19760 E+00$

$17159 E+\theta 0$

$13919 E+\theta \theta$

$.10004 E+00$

53775E-01

$-.44409 E-15$
FP

$.41682 E+00$

$.40045 E+00$

$38625 E+00$

$.35432 E+00$

$32094 E+00$

$28603 E+00$

$24950 E+00$

$21126 E+\theta \theta$

$.17121 E+0 \theta$

$12924 E+\theta 0$

$.10750 E+00$

.85236E-01

$62433 E-01$

$.39074 E-01$

$15142 E-01$

$-.93809 E-02$

$-.34513 E-01$

$-.60272 E-01$

$-.86680 E-01$

$-.11376 E+00$

$-.17000 E+00$

-. $22918 E+00$

-.29151E+00

$-.35718 \mathrm{E}+00$

$-.42643 E+00$

$-.49951 E+00$

$-.57670 E+00$
THETA

$.37774 E+00$

$.38204 E+00$

$38575 E+00$

$.39406 \mathrm{E}+00$

$.40266 \mathrm{E}+00$

$.41159 E+00$

$.42085 E+00$

$43046 E+\theta 0$

$.44045 \mathrm{E}+00$

$.45083 E+00$

$.45617 \mathrm{E}+00$

$.46162 E+00$

$46718 E+00$

$.47285 E+00$

$47864 E+00$

$.48455 E+00$

$.49058 \mathrm{E}+00$

$.49673 E+00$

$.50302 E+00$

$50943 E+00$

$52268 E+00$

$.53652 E+00$

$.55097 E+00$

$56608 E+0 \theta$

$.58188 E+00$

$-59843 E+00$

$.61577 E+00$
THETAP

$.78724 E-01$

$80233 E-01$

$81546 E-01$

$.84509 E-01$

87622E-01

90897E-01

$.94343 E-01$

$97973 E-01$

$.10180 E+00$

$10583 E+00$

$10793 E+00$

$.11009 E+00$

$11231 E+\theta \theta$

$.11459 E+00$

$.11693 E+00$

$11935 E+\theta \theta$

$.12183 E+\theta \theta$

- $12438 E+\theta 0$

$12701 E+00$

$12971 \mathrm{E}+00$

$13536 \mathrm{E}+\theta 0$

$14136 E+\theta \theta$

$14772 E+\theta 0$

$15449 E+\theta \theta$

$.16170 E+\theta 0$

$16937 E+00$

$.17756 \mathrm{E}+00$
RA

0.

$12151 E-01$ 22843E-01 $.47392 E-01$ $73774 E-01$ $.10213 E+00$ $13260 E+00$ $16535 E+00$ $.20056 E+00$ $23843 E+06$ $.25842 E+00$ . $27915 E+\theta B$ $.30065 E+00$ . $32296 E+00$ $34610 E+00$ $.37011 E+00$ $.39502 E+00$ . 42087E+00 $.44770 E+00$ $.47555 E+0 \theta$ $.53447 E+00$ $.59798 E+00$ $66650 E+00$ $74045 E+00$ $.82032 E+00$ $90664 E+00$ $.10000 E+01$ 
ETA

$\begin{array}{r}-1.0000 \\ -.9459 \\ -.9000 \\ -.8500 \\ -.8000 \\ -.7000 \\ -.6000 \\ -.5000 \\ -.4000 \\ -.3000 \\ -.2000 \\ -.1500 \\ -.1000 \\ -.0500 \\ 0.0000 \\ .0500 \\ .1000 \\ .1500 \\ .2000 \\ .2500 \\ .3000 \\ .3500 \\ .4000 \\ .4500 \\ .5000 \\ .5494 \\ .6000 \\ .7000 \\ .8000 \\ 1.9000 \\ 1.0000 \\ \hline\end{array}$

F

0.

$.57895 E-01$

$.10633 E+00$

$.15831 E+00$

- $20941 E+00$

$.30879 E+00$

$.40392 E+00$

$.49419 E+00$

$.57885 E+00$

$.65704 E+00$

$.72772 E+00$

$.75986 E+00$

$.78963 E+00$

$.81684 E+00$

$.84127 E+00$

$.86268 E+00$

$.88079 E+00$

$.89531 E+00$

$.90590 E+00$

$.91220 E+00$

$.91379 E+00$

$.91019 E+00$

$.90087 E+00$

$.88524 E+00$

. 86261E+00

$.83259 E+00$

$.79311 E+00$

$.68462 E+00$

$.52674 E+00$

. 3052टE+00

$.35873 E-07$
FP

$10782 E+01$

1 9622E+01

$.10478 E+01$

. $10310 E+01$

$10131 E+01$

$.97348 E+00$

- $92812 E+00$

$.87599 E+00$

$.81587 E+00$

$.74624 E+00$

$.66524 E+00$

$.61977 E+00$

$.57053 E+00$

$.51714 E+00$

$45916 E+00$

. 39609E+00

. $32736 E+00$

. 25233E+00

$.17027 E+00$

$.80320 E-01$

$-.18480 E-01$

- $12726 E+00$

- $24732 E+00$

- $38019 E+00$

$-.52766 E+00$

$-.68987 E+00$

$-.87515 E+00$

$-.13118 E+01$

-.18692E+01

$-.25941 E+01$

$-.35580 E+01$
THETA

$.61906 E+00$

$.63757 E+80$

$.65402 E+00$

. $67276 E+00$

$69242 E+00$

$.73475 E+00$

$.78160 E+00$

$.83366 E+00$

$.89177 E+00$

$95694 E+\theta 0$

$.10304 E+01$

$10707 E+01$

$.11137 \mathrm{E}+01$

$.11597 E+01$

$12088 E+01$

$.12615 E+01$

$.13180 E+01$

$.13789 E+01$

$.14445 E+01$

$.15154 E+01$

$.15921 E+01$

$.16755 E+01$

$176625+01$

$.18652 E+01$

$19736 E+01$

. $20913 E+01$

- 22239E + 01

. $25299 E+01$

$.29104 E+01$

$.33925 E+01$

$.40171 E+01$
THETAP

RA

$.33374 E+00$

$.35069 E+00-.23885 E-02$

$.36602 E+00-.40204 E-02$

$.38379 E+00-.53285 E-02$

$.40278 E+00-.60828 E-02$

$.44487 E+00-.56217 E-02$

$.49329 E+00 \quad-.19152 E-02$

$.54931 E+00 \quad .59639 E-02$

$.61453 E+00 \quad .19214 E-01$

$.69093 E+00 \quad .39397 E-01$

$.78110 E+00 \quad .68561 E-01$

$.83233 E+00 \quad .87328 E-01$

$.88832 E+00 \quad .10942 E+00$

$.94963 E+\theta 0 \quad .13533 E+\theta \theta$

$10169 E+01 \quad .16562 E+\theta 0$

$.10910 E+01$. $20095 E+00$

$.11726 E+01 \quad .24208 E+00$

$.12629 E+01$. . $28993 E+00$

$.13631 E+01 \quad .34556 E+00$

$14745 E+01 \quad .41024 E+00$

$.15988 E+01 \quad .48547 E+0 \theta$

$.17379 E+01 \quad .57305 E+00$

$.18942 E+01 \quad .67512 E+00$

$.20704 E+01 \quad .79430 E+00$

$.22700 E+01 \quad .93372 E+00$

$24941 E+01 \quad .10952 E+01$

$.27559 E+01 \quad .12895 E+01$

$.33962 E+01 \quad .17851 E+01$

$.42585 E+01 \quad .24864 E+01$

$.54499 E+01 \quad .34994 E+01$

$.71464 E+01 \quad .50000 E+01$ 
ETA

$$
\begin{array}{r}
-1.0000 \\
-.9459 \\
-.9000 \\
-.8500 \\
-.8000 \\
-.7000 \\
-.6000 \\
-.5000 \\
-.4000 \\
-.3000 \\
-.2500 \\
-.2000 \\
-.1500 \\
-.1000 \\
-.0500 \\
0.0000 \\
.0500 \\
.1000 \\
.1500 \\
.2000 \\
.2500 \\
.3000 \\
.3500 \\
.4000 \\
.4500 \\
.5000 \\
.5494 \\
.6000 \\
.7000 \\
.8000 \\
.9000 \\
.9773 \\
.9856 \\
1.0000
\end{array}
$$

0 .

70897E-01

$13065 E+08$

$.19525 E+00$ $.25930 E+00$

$.38557 E+\theta 0$

$.50898 E+00$

$.62897 E+00$

$.74483 E+00$

$.85569 E+00$

$90892 E+00$

$.96046 \mathrm{E}+00$

$10101 E+01$

$.10577 E+01$

$.11030 E+01$

$.11457 \mathrm{E}+01$

$.11856 \mathrm{E}+01$

$12221 E+01$

- $12551 E+01$

$.12838 E+01$

- $13079 E+01$

$13267 E+01$

$.13393 E+01$

$13449 E+01$

$.13423 E+01$

$.13303 E+01$

- $13075 E+01$

. $12710 E+01$

. $11489 E+01$

$93527 E+\theta 0$

$.58166 E+00$

$.15901 E+00$

$10323 E+00$

$.14211 \mathrm{E}-13$
FP

$.13154 E+01$

$13059 E+01$

- $12971 E+01$

- $12867 E+01$

- $12753 E+01$

$12493 E+01$

. $12181 \mathrm{E}+01$

$11806 E+01$

$.11353 E+01$

$.10802 E+01$

$10482 E+01$

$.10128 E+01$

$97352 E+00$

$.92978 E+00$

$.88099 E+00$

$82643 E+00$

$.76525 \mathrm{E}+00$

$.69642 E+\theta 0$

$.61874 E+\theta \theta$

$.53076 E+00$

$.43072 E+\theta 0$

$.31648 E+\theta 0$

$.18542 E+\theta 0$

$.34299 E-\theta 1$

$-.14094 E+00$

$-.34541 E+\theta 0$

$-.58268 E+\theta 0$

$-.86995 E+00$

$-.16186 E+01$

$-.27320 E+01$

$-.44797 E+01$

$-.66052 E+01$

$-.68928 E+01$

$-.74306 E+01$
THETA

$48893 E+00$

$.50686 E+00$

$52294 E+00$

$54145 E+00$

$56107 E+00$

$60403 E+00$

$.65273 E+00$

70827E+00

$.77205 E+00$

$.84585 E+00$

$88721 E+00$

$.93199 E+00$

$98060 E+00$

$10335 E+01$

$.10912 E+01$

$.11544 E+01$

$.12237 E+01$

. $13001 E+01$

$.13846 E+01$

$.14784 E+01$

$.15829 E+01$

$.16998 \mathrm{E}+01$

$.18315 E+01$

$.19803 E+01$

. $21497 E+01$

$.23437 E+01$

$.25646 \mathrm{E}+01$

$.28273 E+01$

$.34929 E+01$

$.44481 E+01$

$.58957 E+01$

$.76041 \mathrm{E}+01$

$.78322 E+01$

$.82570 E+01$
THETAP

. $32156 E+00$

. $34140 E+00$

$.35959 E+0 \theta$

$.38096 E+00$

$.40412 E+\theta 0$

$.45666 E+00$

$.51910 E+0 \theta$

$.59397 E+00$

$.68458 E+00$

$.79538 E+00$

$86017 E+00$

$.93244 E+00$

$.10133 E+01$

$.11042 E+01$

- $12067 E+01$

$13226 E+01$

$.14545 E+01$

$.16050 E+01$

$17778 E+01$

. $19771 E+01$

. $22083 E+01$

$.24782 E+01$

. $27953 E+01$

$31706 E+01$

$.36183 E+01$

$.41571 \mathrm{E}+01$

$.48034 E+01$

$.56149 E+01$

$.78686 E+01$

$.11556 E+02$

$.18049 E+02$

. $26952 E+02$

. $28233 E+02$

$.30677 E+02$
RA

$-.83653 E-02$

$-.15492 E-01$

$-.23258 E-01$

-.31003E-01

$-.46283 E-01$

$-.60971 E-01$

$-.74544 E-01$

$-.86250 E-01$

$-.94993 E-01$

$-.97781 E-01$

$-.99176 E-01$

$-.98858 E-01$

$-.96435 E-01$

$-.91424 E-01$

$-.83229 E-01$

-.71114E-01

$-.54160 E-01$

$-.31215 E-01$

$-.82791 E-03$

$.38847 \mathrm{E}-01$

$.90169 E-01$

. $15618 E+00$

$.24086 E+00$

$.34941 E+00$

$.48879 E+00$

$.66606 E+00$

$.90083 E+00$

$16027 E+01$

$.28472 E+01$

$.51898 E+01$

$.85593 E+01$

$.90536 E+01$

$.10000 E+02$ 
THETA

0 .

$-1.0000$

$-.9459$

$-.9000$

$-.8500$

$-.8000$

$-.7000$

$-.6000$

$-.5000$

$-.4000$

$-.3000$

$-.2500$

$-.2000$

$-.1500$

$-.1000$

$-.0500$

0.0000

.0500

.1000

.1500

.2000

.2500

.3000

.3500

.4000

.4500

.5000

.5494

.5747

.6008

.6259

.6518
. 97206E-01 $17969 E+00$ $.26949 E+\theta 0$ $.35926 \mathrm{E}+00$ $.53866 E+00$ $.71783 E+00$ $.89666 E+00$ $.10750 E+01$ - $12527 E+01$ $.13413 \mathrm{E}+01$ . $14296 \mathrm{E}+01$ $.15176 E+01$ $.16052 E+01$ . 16924E+01 $.17792 E+01$ $.18653 \mathrm{E}+01$ $.19507 E+01$ $.20353 E+01$ . $21188 \mathrm{E}+01$ . $22010 E+01$ . $22817 E+01$ $.23604 E+01$ . $24367 E+01$ $.25098 E+01$ $.25787 E+01$ . $26415 E+01$ $26710 E+01$ -26982E+01 - $27234 E+01$ . $27452 E+01$
$17973 E+\theta 1$

$.17968 E+01$

. $17964 E+01$

- $17958 E+01$

$.17950 E+01$

$17930 E+01$

. 17901E+01

-17862E+01

$.17808 E+01$

$.17733 E+01$

$.17686 \mathrm{E}+01$

$.17631 E+01$

$.17488 \mathrm{E}+01$

$.17397 \mathrm{E}+01$

$.17289 E+01$

$.17003 E+01$

$.16815 E+01$

$.16586 E+01$

- $16304 E+01$

$.15955 E+01$

$.15516 E+01$

. $14960 E+01$

$.14241 E+01$

$.13298 E+01$

- $12048 \mathrm{E}+01$

- $11241 \mathrm{E}+01$

$.10285 \mathrm{E}+01$

$.91131 E+\theta 0$

$.76925 \mathrm{E}+00$
$.17565 E+01$

$17159 E+\theta 1$
68408E-01

$.71862 \mathrm{E}-01$

$.75013 E-01$

$.78694 E-01$

$.82665 E-01$

91601E-01

$.10213 E+\theta 0$

$.11467 E+\theta 0$

$12976 E+\theta 0$

$.14818 E+\theta 0$

$.15895 E+00$

$.17098 E+00$

$.18449 E+\theta 0$

$19972 E+\theta 0$

$.21699 E+\theta 0$

$.23668 E+00$

$.25928 E+\theta 0$

$.28539 E+00$

. $31580 E+00$

$.35153 E+\theta 0$

$.39390 E+\theta 0$

$.44469 E+\theta 0$

$.50634 E+00$

.58222E+00

$.67714 E+\theta 0$

$.79816 E+00$

$.95390 E+00$

$.10523 E+01$

$.11673 E+01$

$13062 E+01$

$.14723 E+01$
THETAP

$61474 E-01$

$.66366 \mathrm{E}-01$

. 70940E-01

$.76423 E-01$

$.82494 E-01$

$96750 E-01$

$.11455 \mathrm{E}+0 \theta$

$.13709 E+\theta 0$

$16607 \mathrm{E}+\theta 0$

$.20399 E+00$

. $22741 E+\theta 0$

. $25462 E+\theta \theta$

. $28642 E+00$

$.32383 E+\theta 0$

$.36816 \mathrm{E}+00$

$.42109 E+00$

$48484 E+\theta 0$

$.56234 E+\theta 0$

$65754 E+\theta 0$

$.77585 E+00$

$.92479 E+00$

$.11150 E+01$

$.13620 E+01$

$16889 E+01$

. $21310 E+01$

. $27443 E+01$

. $36094 E+01$

$41971 E+01$

$.49212 E+01$

$.58485 E+01$

$.70270 E+01$
RA

-.30229E-02

$-.57079 E-02$

$-.87661 E-02$

$-.11974 E-01$

$-.18883 E-01$

$-.26539 E-01$

$-.35069 E-01$

$-.44627 E-01$

-.55402E-01

$-.61319 E-01$

$-.67631 E-01$

$-.74379 E-01$

$-.81603 E-01$

$-.89351 E-01$

$-.97672 E-01$

$-.10662 E+00$

$-.11625 E+0 \theta$

$-.12663 E+00$

$-.13779 E+\theta 0$

$-.14978 E+0 \theta$

$-16262 E+\theta 0$

$-.17623 E+80$

$-.19046 E+00$

- $20493 E+00$

$-.21882 E+00$

$-.23040 E+00$

- $23458 E+\theta \theta$

$-.23673 E+00$

$-.23579 E+\theta 0$

$-.23009 E+00$ 


\begin{tabular}{|c|c|c|c|c|c|}
\hline $\begin{array}{l}.6972 \\
.7000 \\
.7202 \\
.7378 \\
.7715 \\
.8000 \\
.8692 \\
.8793 \\
.9000 \\
.9434 \\
.9503 \\
.9603 \\
.9773 \\
.9856 \\
.9963 \\
.9989 \\
.0000\end{array}$ & $\begin{array}{l}.27731 E+01 \\
.27743 E+01 \\
.27806 E+01 \\
.27825 E+01 \\
.27744 E+01 \\
.27514 E+01 \\
.25884 E+01 \\
.25441 E+01 \\
.24251 E+01 \\
.19722 E+01 \\
.18573 E+01 \\
.16557 E+01 \\
.11727 E+01 \\
.84213 E+00 \\
.25696 E+00 \\
.80198 E-01 \\
.56843 E-13\end{array}$ & $\begin{array}{r}.43625 E+\theta 0 \\
.41148 E+\theta 0 \\
.21151 E+\theta \theta \\
.21338 E-\theta 2 \\
-.51490 E+\theta 0 \\
-.11317 E+\theta 1 \\
-.40499 E+\theta 1 \\
-.47931 E+\theta 1 \\
-.68234 E+\theta 1 \\
-.15502 E+\theta 2 \\
-.17986 E+\theta 2 \\
-.22639 E+\theta 2 \\
-.35313 E+\theta 2 \\
-.45241 E+\theta 2 \\
-.65325 E+\theta 2 \\
-.72022 E+\theta 2 \\
-.75255 E+\theta 2\end{array}$ & $\begin{array}{l}.18539 E+01 \\
.18820 E+01 \\
.21070 E+01 \\
.23403 E+01 \\
.29085 E+01 \\
.35757 E+01 \\
.66482 E+01 \\
.74174 E+01 \\
.95025 E+01 \\
.18265 E+02 \\
.20747 E+02 \\
.25376 E+02 \\
.37902 E+02 \\
.47657 E+02 \\
.67301 E+02 \\
.73832 E+02 \\
.76984 E+02\end{array}$ & $\begin{array}{l}.10005 E+02 \\
.10237 E+02 \\
.12171 E+02 \\
.14293 E+02 \\
.19931 E+02 \\
.27328 E+02 \\
.70399 E+02 \\
.83179 E+02 \\
.12129 E+03 \\
.32745 E+03 \\
.39733 E+03 \\
.53934 E+03 \\
.99069 E+03 \\
.14014 E+04 \\
.23624 E+04 \\
.27175 E+04 \\
.28967 E+04\end{array}$ & $\begin{array}{r}-.20058 E+00 \\
-.19759 E+00 \\
-.17001 E+00 \\
-.13499 E+00 \\
-.25310 E-01 \\
.14196 E+00 \\
.13237 E+01 \\
.17060 E+01 \\
.28861 E+01 \\
.96869 E+01 \\
.12061 E+02 \\
.16935 E+02 \\
.32663 E+02 \\
.47127 E+02 \\
.81224 E+02 \\
.93875 E+02 \\
.10000 E+03\end{array}$ \\
\hline
\end{tabular}




\section{REFERENCES}

1. R. A. Wooding, "Convection in a Saturated Porous Medium at Large Rayleigh or Peclet Number," J. Fluid Mech., 15, pp. 527-546, 1963.

2. H. Schlichting, Boundary Layer Theory, 6 th Edition, McGraw-Hill, New York, NY, 1965 .

3. C. E. Hickox and H. A. Watts, "Steady Thermal Convection from a Concentrated Source in a Porous Medium," SAND76-0562, Sandia Laboratories, Albuquerque, NM, 1976 .

4. C. S. Yih, Dynamics of Nonhomogeneous Fluids, Macmillan Co., New York, NY, 1965 .

5. H. B. Squire, "The Round Laminar Jet," Quart. J. Mech. Appl. Math. , 4 , pp. $321-329,1951$.

6. A. Bejan, "Natural Convection in an Infinite Porous Medium with a Concentrated Heat Source," J. Fluid Mech., 89, Part 1, pp. 97-101, 1978.

7. M. R. Scott and H. A. Watts, "A Systematized Collection of Codes for Solving Two-Point Boundary Value Problems," in Numerical Methods for Differential Systems-Recent Developments in Algorithms, Software, and Applications, L. Lapidus and W. Schiesser, Eds., Academic Press, New York, NY, 1976.

8. M. R. Scott and H. A. Watts, "Computational Solution of Nonlinear Two-Point Boundary Value Problems," Proc. 5 th Symposium of Computers in Chem. Engr., Czechoslovakia, 1977, also SAND77-0091, Sandia Laboratories, Albuquerque, NM, 1976 . 
DISTRIBUTION :

Oregon State University

School of Oceanography

Corvalis, OR 97331

G. R. Heath

Harvard University

Pierce Hall

Cambridge, MA

A. Robinson

Applied Physics Laboratory

University of Washington

1013 Northeast Fortieth Street

Seattle, WA 98105

T. E. Ewart

Cornell University

254 Upson Hall

Sibley School

Mechanical and Aerospace Engineering

Ithaca, NY 14853

P. R. Dawson

University of Rhode Island

Department of Civil Engineering

Kingston, RI 02881

A. J. Silva

Falcon R\&D

2350 Alamo Avenue, SE

Albuquerque, NM 87106

D. W. Jackson

Woods Hole Oceanographic Institution Redfield Building, 3-32

Woods Hole, MA 02543

C. D. Hollister

U.S. Department of Energy

ONWI Library

Battelle Columbus Laboratory

505 King Avenue

Columbus, OH 43201 Beverly Rawles
Sandia Internal:

2600 L. E. Hollingsworth

2640 J. L. Tischhauser

2646 M. R. Scott

2646 H. A. Watts (5)

2646 L. A. Bertram

2646 A. H. Treadway

4004 M. J. Becktell

4500 E. H. Beckner

4530 R. W. Lynch

4536 D. R. Anderson

4536 L. Brush

4536 L. S. Gomez

4536 R. D. Klett

4536 J. Lipkin

4536 C. M. Percival (5)

4536 D. M. Talbert

4723 W. P. Schimmel

4743 H. C. Hardee

5500 O. E. Jones

5510 D. B. Hayes

5511 J. W. Nunziato

5511 D. K. Gartling

5511 C. E. Hickox (25)

5512 D. F. McVey

5512 R. H. Nilson

5513 D. W. Larson

5520 T. B. Lane

5521 M. G. Marietta

5530 W. Herrmann

5531 P. F. Chaves

5531 W. T. Brown

5541 J. L. Krumhans1

5620 M. M. Newsom

5812 K. L. Erickson

5831 J. W. Braithwaite

5835 C. H. Karnes

5840 N. J. Magnani

8266 E. A. Aas

3141 T. L. Werner (5)

3151 W. L. Garner (3)

For: DOE/TIC (Unlimited Release)

DOE/TIC (25)

(R. P. Campbel1, 3154-3) 